Characterization of optically and chemically stimulated neurotransmitter release in Drosophila melanogaster

\author{
Poojan Pyakurel \\ Kathmandu, Nepal
}

B.S., Randolph College, 2011

\begin{abstract}
A Dissertation presented to the Graduate Faculty of the University of Virginia in Candidacy for the Degree of Doctor of Philosophy
\end{abstract}

Department of Chemistry

University of Virginia

July, 2017 


\section{Acknowledgements}

I would like to express my deepest gratitude to my research advisor, Dr. Barbara Jill Venton, for her immense support and guidance to help me grow as a research scientist. Working with her has polished my scientific writing skills greatly and without her this dissertation would not have been possible. I would also like to thank my dissertation committee members for their time and support throughout my years in the department. Special thanks to Dr. Jay Hirsh for sharing his knowledge on Drosophila and for donating Drosophila stocks.

I would also like to thank my family and friends for their love and support. Thank you to my parents, my husband, and my sisters for their continuous encouragement for me to succeed. Thanks to all my friends for their precious company, especially friends from Venton lab for making my time in the lab special and for productive discussion about research. Finally, I would like to thank the staff and faculty at the Department of Chemistry at UVA for working together to create a wonderful work environment, and for their prompt help with ordering, maintenance and completion of paperwork. 


\title{
Characterization of optically and chemically stimulated neurotransmitter release in Drosophila melanogaster
}

\author{
Abstract \\ Drosophila melanogaster is a popular model organism to study \\ neurotransmission and neurological disorders. There is a wide range of genetic \\ manipulation tools available for use in Drosophila, which has homologs for $75 \%$ \\ of human disease genes. In addition, several fundamental neurological \\ processes are conserved between the two species. Drosophila is therefore an \\ excellent model organism for high throughput screening of genes involved in \\ diseases and of drugs that can be used to treat the diseases. Since many \\ neurological diseases are caused by changes in neurotransmitters, it is \\ imperative to understand the fundamentals of neurochemistry and how it \\ changes during a disease process. Electrochemical methods have long been \\ used to measure neurotransmitters and their dynamics in the brain. Many \\ neurotransmitters such as dopamine and octopamine are electroactive and can \\ be measured directly on electrochemical sensors as described in this thesis. \\ Fast-scan cyclic voltammetry (FSCV) is a method that can provide rapid, \\ sensitive, and selective measurements of neurotransmitters in the brain. This \\ thesis describes new methods to stimulate endogenous dopamine release in \\ Drosophila larval and adult CNS, and optimization of the FSCV waveform for \\ measurements of endogenous octopamine in larvae. Chapter 2 describes \\ acetylcholine, nicotine, and neonicotinoid stimulated dopamine release in
}


Drosophila larval VNC. Since acetylcholine acts on endogenous Drosophila receptor nAChR to stimulate dopamine release, this method can be used to study release in most Drosophila lines. The method was also used to make the first measurements of evoked dopamine release in adult Drosophila brains in our lab. Chapter 3 describes stimulated dopamine and dopamine tissue content measurements in the CNS of adult controls and Parkinson's disease model Drosophila with a knockdown of Parkin or RNF11 with RNAi. We found that aging does not affect the concentration of stimulated dopamine release, or the tissue content in the CNS, but has an effect when stimulations are repeated at short intervals. The release in old adults declines significantly slower than in mid-age adults. This effect was lost in Parkinson's disease mutants suggesting that the mutations change dopamine dynamics in old adults. In Chapter 4, I describe optimization of the FSCV waveform for the detection of octopamine in situ. The new waveform uses a higher switching potential and a slower scan rate than the previously developed waveform for octopamine detection in vitro. With the new waveform, octopamine oxidation peak is detected away from the switching potential, where the background signal is most unstable. The waveform was used to measure and characterize light or ATP mediated octopamine release in Drosophila larval VNC. The methods described in this thesis enable measurements of stimulated dopamine release in most Drosophila larvae and adults, and stimulated octopamine release in larvae expressing transgenic ion 
channels. Future studies can investigate the effects of disease or other mutations on the release and clearance of these neurotransmitters. 


\section{Table of Contents}

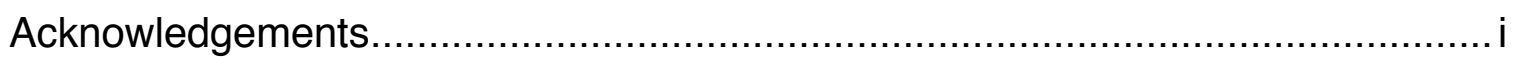

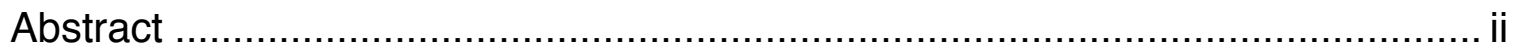

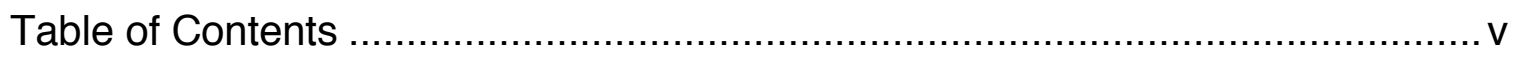

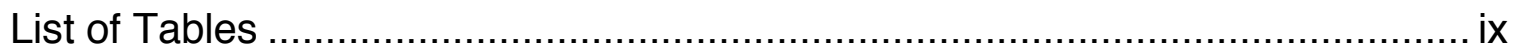

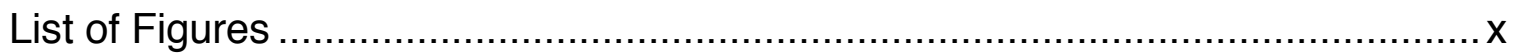

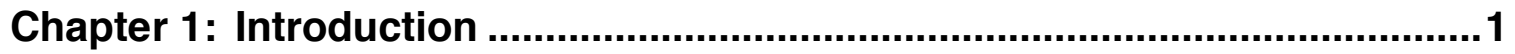

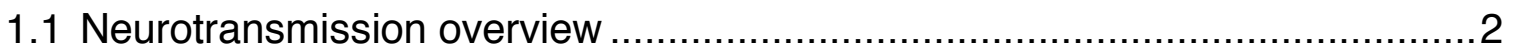

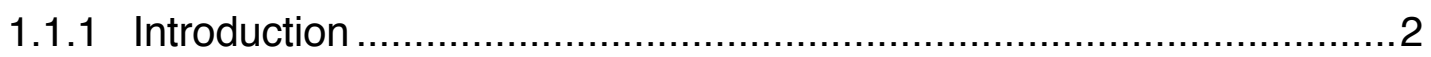

1.2 Drosophila melanogaster - a model organism ....................................... 4

1.2.1 Galactosidase-4-upstream activating sequence (GAL4-UAS) ............6

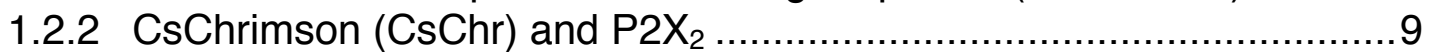

1.2.3 Neurotransmitter measurement in life stages of Drosophila ................ 9

1.3 Neurotransmitters dopamine, octopamine and acetylcholine......................11

1.3.1 Effect of insecticides on insect neurotransmitters...........................15

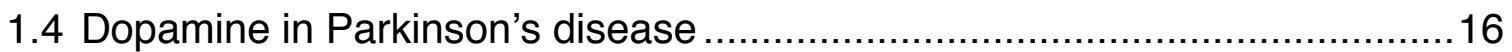

1.4.1 Parkinson disease model Drosophila melanogaster........................17

1.5 Electrochemical detection of neurotransmitters .........................................18

1.5.1 Introduction to electrochemistry ..............................................18

1.5.2 Carbon fiber microelectrode for electrochemical detection ................19

1.5.3 Electrochemical detection of neurotransmitters at CFMEs ................20

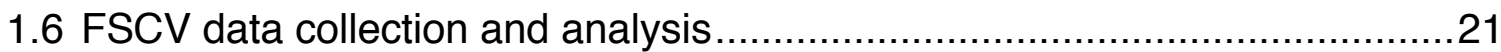

1.6.1 Redox mechanisms for dopamine and octopamine .........................23

1.6.2 FSCV waveform optimization ...................................................24

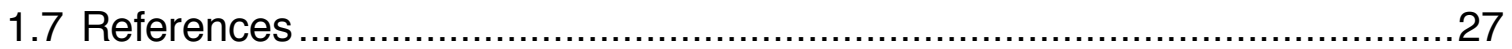

Chapter 2: Nicotinic acetylcholine receptor (nAChR) mediated dopamine release in larval Drosophila melanogaster.................................39

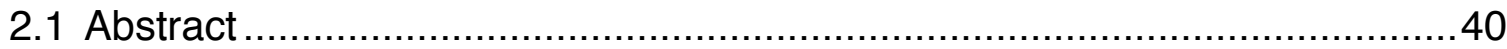




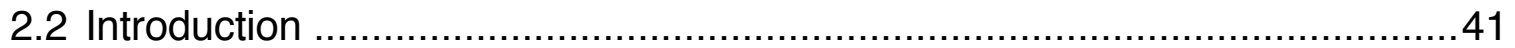

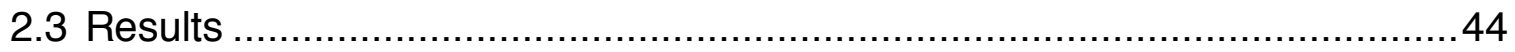

2.3.1 Acetylcholine stimulates dopamine release in Drosophila melanogaster larval VNC .............................................................. 44

2.3.2 Acetylcholine-stimulated release is mediated by $\mathrm{nAChR}$ and not mAchR ................................................................................... 46

2.3.3 Nicotine-stimulated dopamine release in Drosophila melanogaster

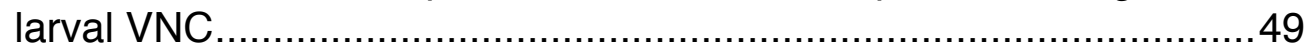

2.3.4 Neonicotinoid-stimulated dopamine release in Drosophila melanogaster larval VNC ...........................................................52

2.3.5 Nicotine and neonicotinoid stimulated release in $\alpha$ and $\beta$ nAChR subunit mutants .....................................................................5

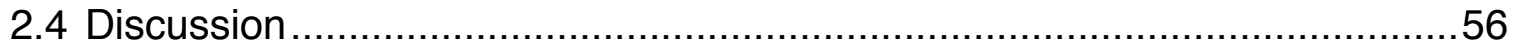

2.4.1 Acetylcholine-stimulated dopamine release …...............................56

2.4.2 Nicotine-stimulated dopamine release ..........................................59

2.4.3 Neonicotinoid-stimulated release ................................................61

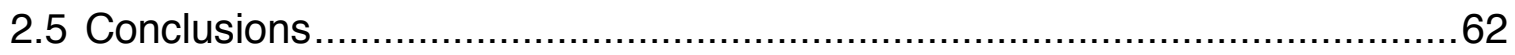

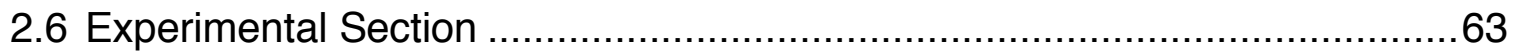

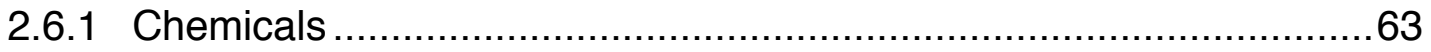

2.6.2 Drosophila and VNC Preparation ................................................64

2.6.3 Electrochemical Setup and Data Analysis ......................................65

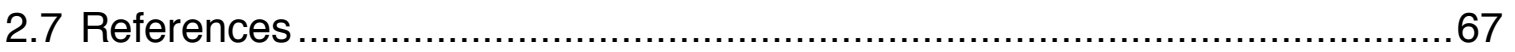

Chapter 3: Age-related changes in Drosophila melanogaster controls and Parkin or RNF-11 mutants ............................................................73

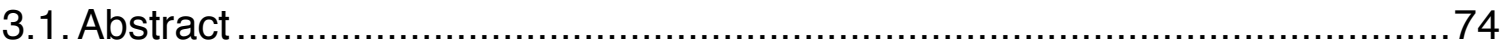

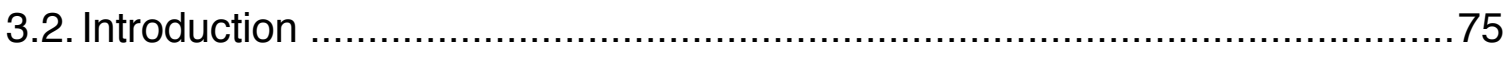

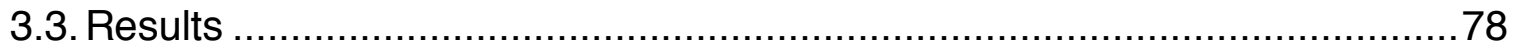

3.3.1 Acetylcholine-stimulated dopamine release in Drosophila

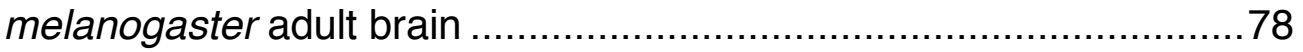

3.3.2 Stimulated dopamine and dopamine tissue content with age .............80

3.3.3 Stimulated dopamine and dopamine tissue content with age in Parkin

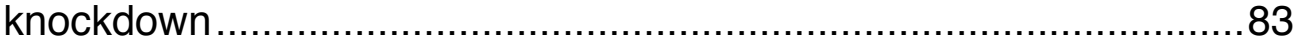

3.3.4 Stimulated dopamine and dopamine tissue content with age in RNF11

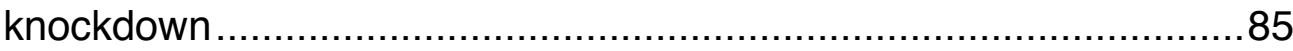

3.3.5 Comparison of release with repeated stimulations in different lines ..87 
3.4. Discussion

3.4.1 Acetylcholine stimulated dopamine release in Drosophila melanogaster adult brain

3.4.2 Stimulated dopamine release and dopamine tissue content during aging in Drosophila....

3.4.3 Stimulated dopamine release and dopamine tissue content in Parkin knockdown Drosophila.

3.4.4 Stimulated dopamine release and dopamine tissue content in RNF11 knockdown Drosophila. .94

3.4.5 Repeated stimulations compared in different Drosophila lines ..........96

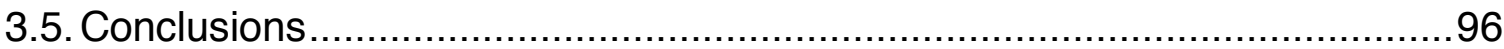

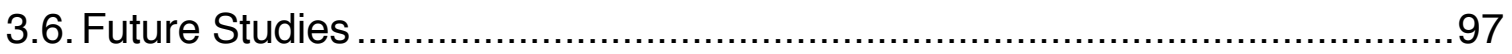

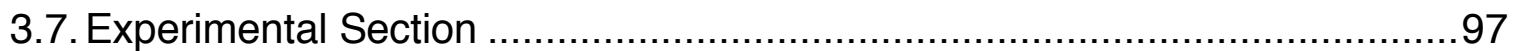

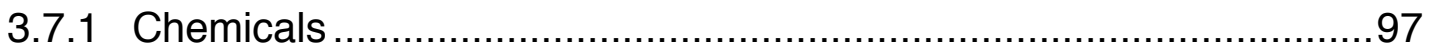

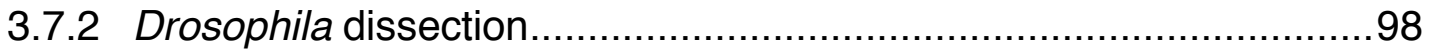

3.7.3 Drosophila homogenate preparation ...........................................99

3.7.4 Instrumentation and data analysis ...............................................99

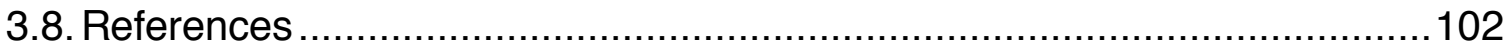

Chapter 4: Fast-scan cyclic voltammetry (FSCV) detection of endogenous octopamine in Drosophila melanogaster ventral nerve cord ..108

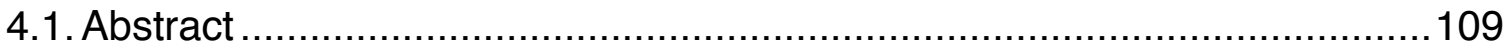

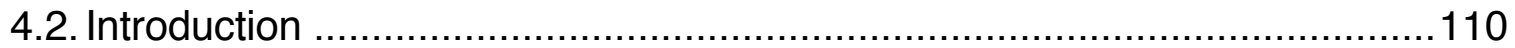

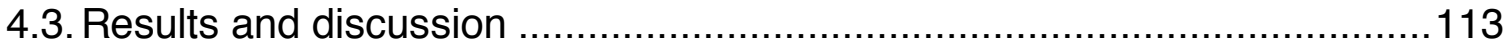

4.3.1 Visualization of octopaminergic neurons .......................................113

4.3.2 Optimization of FSCV waveform for octopamine detection .............114

4.3.3 Confirmation the released compound is octopamine and that release is vesicular

4.3.4 Comparison of puffed on, CsChrimson, and P2X2-mediated octopamine release

4.3.5 Stability of release and effect of stimulation frequency ...................121

4.3.6 Characterization of octopamine clearance ....................................125

4.3.7 Comparison of octopamine release with dopamine and serotonin .. 127

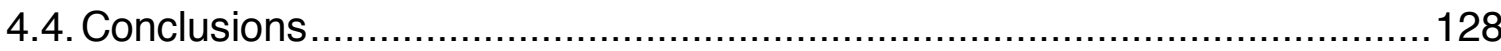

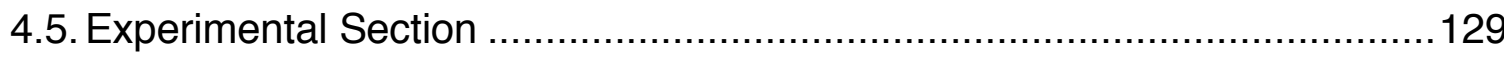




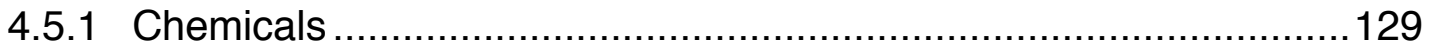

4.5.2 Drosophila and VNC preparation ................................................130

4.5.3 Electrochemical setup, light stimulation, and data analysis.............131

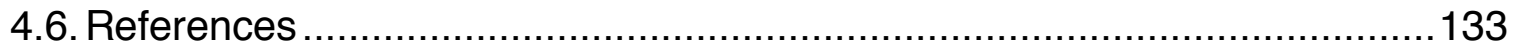

Chapter 5: Conclusions and Future Directions ........................................138

5.1. nAChR mediated dopamine release in Drosophila melanogaster..............140

5.2. Evoked dopamine release in adult Drosophila and in Parkinson's disease

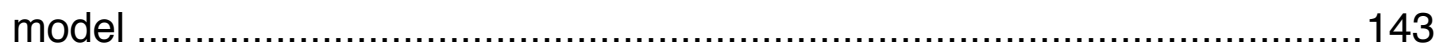

5.3. Optimization of a FSCV waveform for in situ detection of octopamine .......147

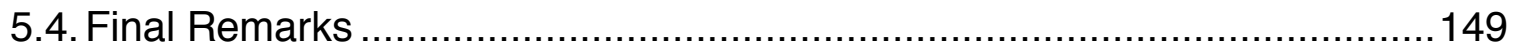

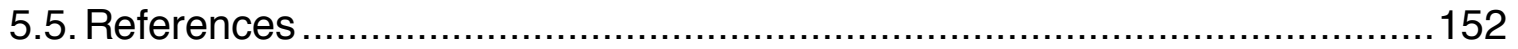

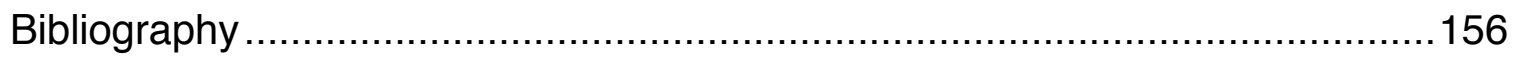




\section{List of Tables}

Table 1. Effect of uptake inhibitors on octopamine release and clearance .......126 


\section{List of Figures}

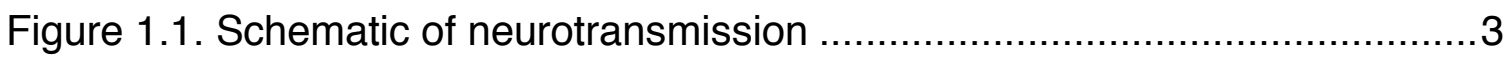

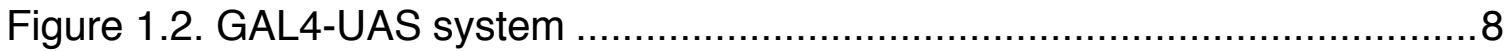

Figure 1.3. Life cycle of Drosophila melanogaster........................................11

Figure 1.4. Chemical structures of neurotransmitters studied here ....................12

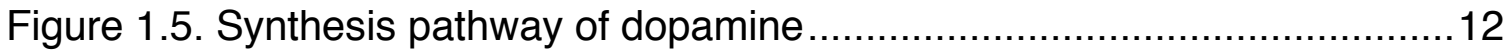

Figure 1.6. Octopamine synthesis pathway ..................................................14

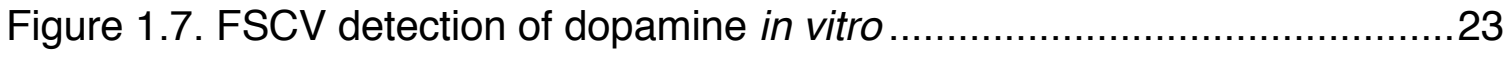

Figure 2.1. Acetylcholine-stimulated dopamine release …..............................45

Figure 2.2. Acetylcholine-stimulated dopamine release is mediated by nAChR .48

Figure 2.3. Nicotine stimulates dopamine release .........................................50

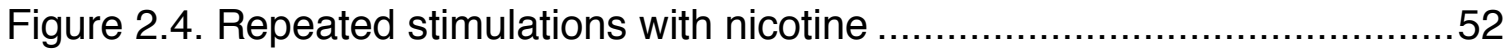

Figure 2.5. Neonicotinoids nitenpyram and imidacloprid stimulated release .......54

Figure 2.6. Effect of nAChR mutations on nicotine and neonicotinoid-stimulated

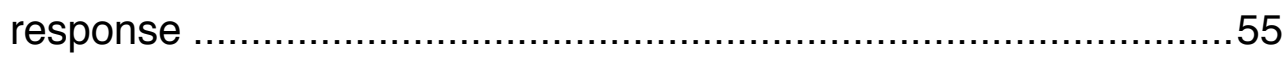

Figure 3.1. Acetylcholine-stimulated dopamine in the central complex region of Drosophila melanogaster brain ..................................................

Figure 3.2. Dopamine release with increasing acetylcholine stimulation.............80

Figure 3.3. Acetylcholine-stimulated dopamine release in Drosophila melanogaster brain and tissue content in the CNS

Figure 3.4. Acetylcholine-stimulated dopamine release in Drosophila melanogaster brain and tissue content in the CNS .84

Figure 3.5. Acetylcholine-stimulated dopamine release in Drosophila melanogaster brain and tissue content in the CNS .86

Figure 3.6. Acetylcholine-stimulated release in control, Parkin-RNAi, and RNF11RNAi Drosophila.. .88

Figure 4.1. Visualization of neurons expressing tyrosine decarboxylase 114 
Figure 4.2. Optimization of FSCV waveform for octopamine detection .............115

Figure 4.3. Confirmation of the detection of octopamine ................................118

Figure 4.4. Comparison of puffed-on octopamine and different stimulations ....121

Figure 4.5. Stability of CsChrimson stimulated octopamine release .................122

Figure 4.6. Effects of length of continuous light stimulation and frequency of pulsed stimulations...................................................................123

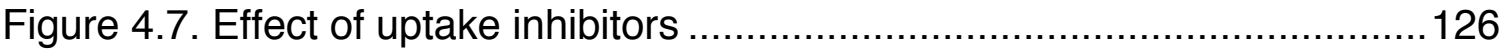


Chapter 1: Introduction 


\section{Chapter 1: Introduction}

\subsection{Neurotransmission overview}

\subsubsection{Introduction}

Brain cells, or neurons communicate with each other by means of electrical and chemical signals. Neurons are activated by an action potential, which occurs when there is an influx of sodium ions into the neuron making the inside of the neuron more positively charged than the extracellular space. Voltage-gated ion channels on the membrane of the neurons regulate ion flow across the cell and hence are responsible for activating and deactivating neurons by flow of mainly $\mathrm{Na}^{+}, \mathrm{K}^{+}, \mathrm{Ca}^{2+}$ and $\mathrm{Cl}^{-}$ions. A neuron can pass a signal to another neuron by releasing neurotransmitters at the synapse, the space between two neurons. Major neurotransmitters in vertebrates are acetylcholine, dopamine, serotonin, epinephrine, norepinephrine, glutamate and GABA. Invertebrates also use the same neurotransmitters except they have tyramine and octopamine instead of epinephrine and norepinephrine.

Neurotransmitters are synthesized and stored in vesicles in neurons (Fig. 1.1). After neuronal firing, they are released from the presynaptic neuron into the synapse. They interact with receptor sites at the postsynaptic cells, which receive and propagate the signal. When a system of neurons, which is a group of neurons expressing one particular neurotransmitter are activated, a bigger volume of the brain rather than a single synapse is activated. This is called volume transmission and is caused when extracellular fluid containing the neurotransmitter diffuses to the extrasynaptic space. ${ }^{1}$ Once released into the 
synapse, the neurotransmitters can be metabolized, taken back up by the presynaptic cell through transporters (reuptake), or diffuse away.

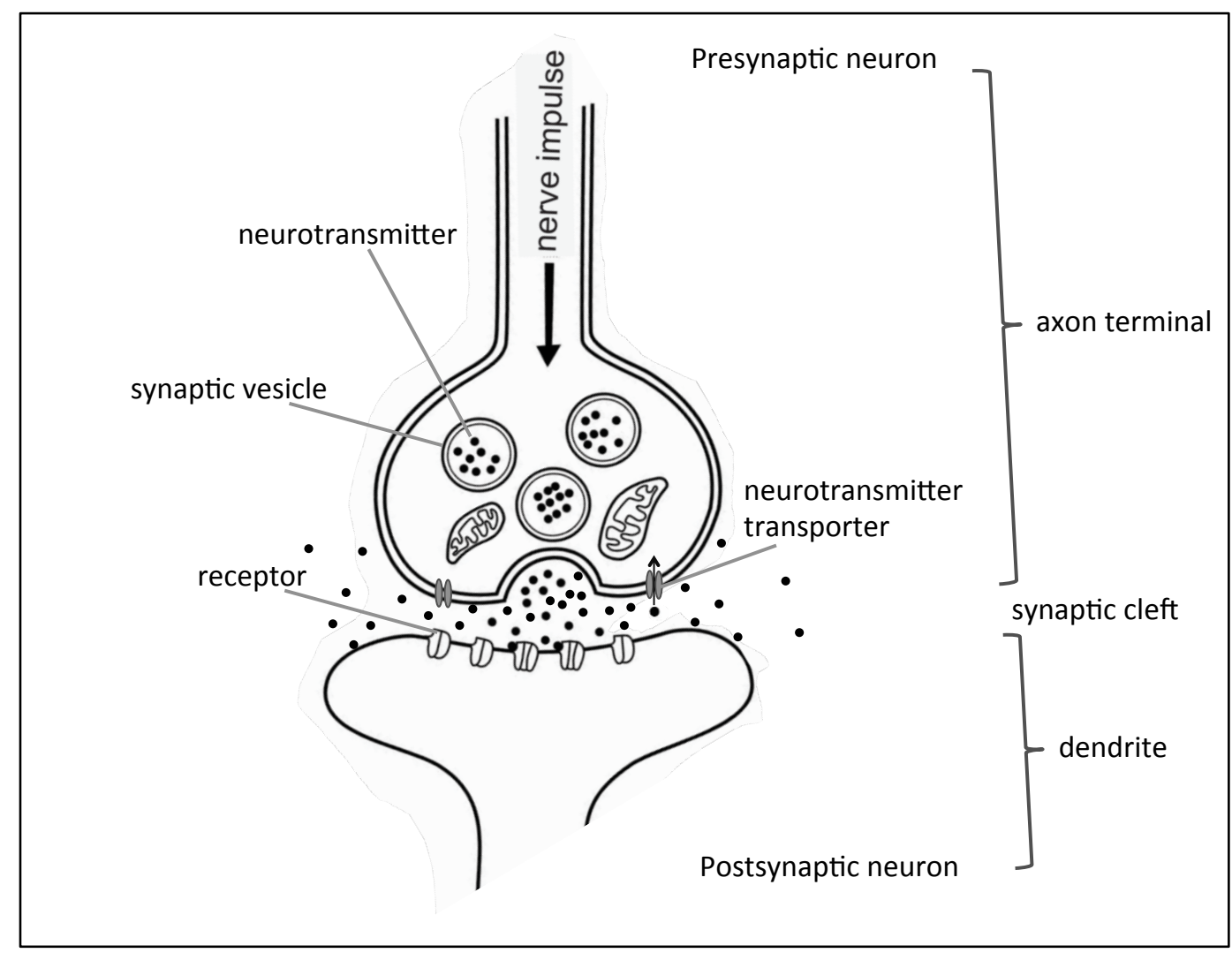

Figure 1.1. Schematic of neurotransmission. The presynaptic neuron synthesizes and stores neurotransmitters in synaptic vesicles. Upon neuronal firing, the vesicle fuses to the membrane releasing neurotransmitters into the synaptic cleft and activating the receptors on the postsynaptic membrane. Neurotransmitters can diffuse away from the synapse and activate neurons at extrasynaptic space, a process known as volume transmission.

Changes in one or more of these events of neurotransmission have been seen in several disorders. Alzheimer's disease is associated with acetylcholine deficiency. ${ }^{2}$ Serotonin imbalance is found in depression ${ }^{3}$ and impulsive behaviors, ${ }^{4}$ and norepinephrine imbalance is seen with depression and ADHD. ${ }^{5}$ There is a loss of dopaminergic neurons in the substantia nigra in Parkinson's 
disease,${ }^{6}$ increased expression of dopamine transporters in $\mathrm{ADHD}^{7,8}$ and dopamine dysregulation in schizophrenia. ${ }^{9}$

As changes in the levels of neurotransmitters and their regulation can cause various disorders, it is important to understand how their levels change and in what ways they are regulated during a disorder. The concentration of neurotransmitters released in the synapse is in the nanomolar-micromolar range,${ }^{10,11}$ and neurotransmitters are cleared rapidly in within milliseconds. ${ }^{12,13}$ Therefore, a fast and sensitive method is required to study neurotransmission. In addition, to study small brain structures, a method with high spatial resolution is needed. Finally, since the brain is a complex structure with many electroactive compounds, the method also needs to be selective. Using Drosophila melanogaster model organism, I have studied different neurotransmitters in the central nervous system of the insect with a rapid, sensitive and selective electrochemical method, fast-scan cyclic voltammetry (FSCV). This introduction will describe Drosophila as a model organism; neurotransmitters dopamine, octopamine and acetylcholine; fast-scan cyclic voltammetry (FSCV) at carbon fiber microelectrode (CFME); and optimization of FSCV for neurotransmitter detection.

\subsection{Drosophila melanogaster - a model organism}

The discovery of the white gene that causes the white eye phenotype in Drosophila melanogaster, or fruit flies, eventually led to discoveries of the role of 
chromosome on heredity, and earned biologist Thomas Hunt Morgan the Novel Prize in Physiology. ${ }^{14}$ Since this discovery in the early 1900s, Drosophila has been a popular model organism to study the effects of gene mutations from molecular ${ }^{15}$ to behavioral ${ }^{16}$ levels. A short life span, easy and low cost maintenance, fully sequenced genome and ease of genetic manipulation are some of the reasons for the popularity of Drosophila as a model organism. ${ }^{17}$ Even though the fruit fly genome is much smaller than the human genome, many fundamental neurological processes are conserved between humans and Drosophila. ${ }^{18}$ In addition, $75 \%$ of human disease genes have a homology in fruit flies, which makes it an important model organism to study human diseases. ${ }^{19}$ Research in Drosophila has answered fundamental questions about sleep, ${ }^{20,21}$ Parkinson's disease,${ }^{22-24}$ and aggression ${ }^{25}$ to give a few examples.

A wide variety of sophisticated genetic tools are available for Drosophila, more than any other multicellular organism. ${ }^{26}$ The ability to create small changes in the genotype by adding or removing small portions of genes, or changing the levels of gene expression makes Drosophila an excellent model organism to understand the effect of small changes in the genome. Forward genetics has been a popular method to understand the genotype of diseases in Drosophila. It is a method where random mutations are created and the genotype that results in a particular phenotype is identified. Chemical mutations using ethyl methane sulfonate (EMS), ${ }^{27}$ insertional mutations with transposable elements, ${ }^{28}$ or expression of RNAi under GAL4-UAS have been used for forward genetics in 
Drosophila. ${ }^{29}$ The GAL4-UAS system was extensively used in my research and is described in detail below.

\subsubsection{Galactosidase-4-upstream activating sequence (GAL4-UAS)}

There are several genetic technologies available to precisely manipulate gene expression and study the downstream effects in Drosophila. Among the various toolkits available, the GAL4- upstream activating sequence (GAL4-UAS) system was the first technique used for the targeted expression of certain genes in specific cell or tissue type. ${ }^{30}$ The GAL4-UAS system, described as "a fly geneticist's swiss army knife" ${ }^{31}$ is a technique used to express specific proteins in a specific cell or tissue type. The gene GAL4, originally discovered in yeast, codes for the GAL4 protein. GAL4 can bind to and activate another gene, the upstream activating sequence (UAS), which in turn transcribes any gene under the control of UAS. As shown in Figure 1.2A, GAL4-UAS is a two-part activation system, which by crossing parent flies with GAL4 and UAS, can be used for restricted expression of genes in the nervous system of Drosophila. An example of using the system to express GFP in the dopaminergic neurons in a Drosophila brain is shown (Fig. 1.2B). Flies with GAL4 linked to dopamine synthesis enzyme tyrosine hydroxylase (TH), (TH-GAL4), are crossed with ones with UAS-GFP. In the offspring, only dopaminergic cells express GFP upon stimulation. TH-GAL4; UAS-GFP Drosophila brains show that there are different clusters of dopaminergic cells that project to different regions in the brain. The GAL4-UAS system can similarly be used to express proteins such as ion channels in specific 
cell types such as dopaminergic cells. Here, the GAL4-UAS system has been used to express cation channels CsChrimson and $\mathrm{P}_{2} \mathrm{X}_{2}$ (described below) in octopaminergic cells to study stimulated octopamine release.

GAL4-UAS system can also be used to knockdown specific proteins by using RNA interference (RNAi). RNAi is a process where cells silence an unwanted gene. The process is triggered when double-stranded RNA (dsRNA) is cleaved into smaller fragments called small interfering RNA (siRNA). ${ }^{32}$ The siRNA bind to RNA-induced silencing complex (RISC), which separates the two strands, one of which is transported to the mRNA. The mRNA is hence silenced and gene is transcription is reduced. 


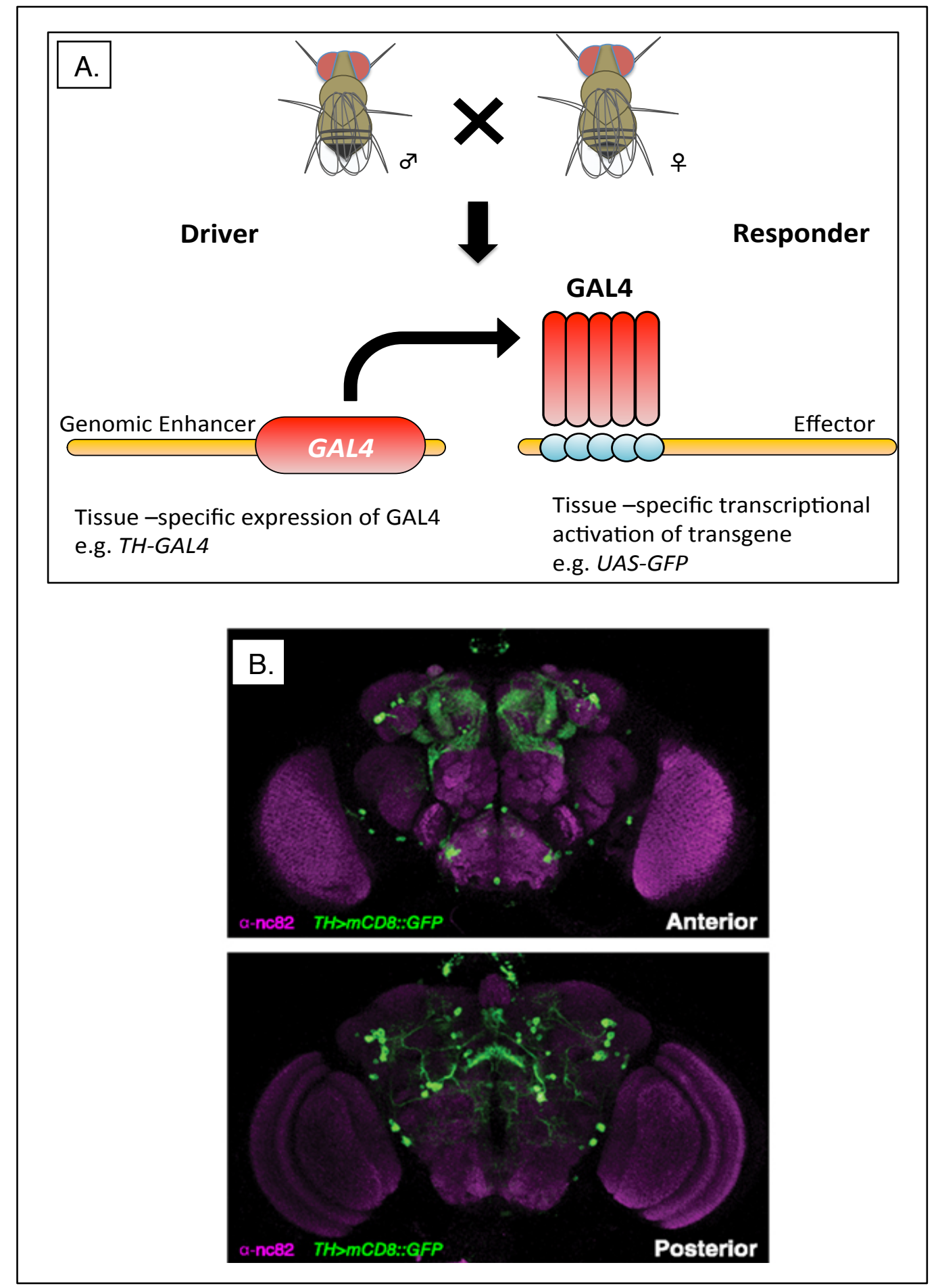

Figure 1.2. GAL4-UAS system. (A) When males carrying a GAL4 driver are crossed with virgin females carrying a UAS responder, the offspring contains both the driver and the responder. (B) Image of adult Drosophila brain expressing GFP on dopaminergic neurons (UAS-mCD8::GFP; TH1Gal4) (reprinted from White K.E. et al., 2010). ${ }^{2}$ 


\subsubsection{CsChrimson (CsChr) and ${\mathrm{P} 2 \mathrm{X}_{2}}$}

CsChr is a non-selective cation channelrhodopsin activated by red light. CsChr has several advantages to a blue light sensitive channelrodopsin (ChR2) that was previously used in our lab. ${ }^{33,34}$ Red light has less energy, which causes less tissue damage and photoelectric effect. ${ }^{35}$ It also travels deeper into tissue than blue light. CsChr also has faster turn-on, turn-off, and recovery kinetics allowing for more precise control of stimulation. ${ }^{36}$ When cells expressing CsChr are stimulated by red light, the channel opens up allowing cations, mostly $\mathrm{Na}^{+}$ into the cell, which depolarizes the cell causing an action potential and release of neurotransmitters.

$\mathrm{P} 2 \mathrm{X}_{2}$ belongs to a class of ligand-gated purinergic receptors that is activated by ATP. ${ }^{37}$ Once bound to ATP, the channel opens up allowing cations to enter the cell and depolarizing it. ${ }^{38} \mathrm{P} 2 \mathrm{X}_{2}$ is also not found in Drosophila and can be genetically inserted into its genome. ${ }^{39,40}$ When ATP is puffed on for activation, $\mathrm{P} 2 \mathrm{X}_{2}$ channels bind to the molecules changing the channel confirmation, causing the channel to open and cells to depolarize. By using the GAL4-UAS system to express the channels CsChrimson (CsChr) or $\mathrm{P}_{2} \mathrm{X}_{2}$ in octopaminergic cells, I have measured stimulated octopamine for the first time in Drosophila larva as described in Chapter 3 of this thesis.

\subsubsection{Neurotransmitter measurement in life stages of Drosophila}

The life cycle Drosophila melanogaster has four main stages - embryo, larva, pupa and adult (Fig. 1.3). The central nervous system (CNS) starts 
developing at the embryonic stage where neurons and glial cells are present. ${ }^{41}$ The CNS continues to grow and mature till adulthood and the neurotransmitter contents change throughout the life cycle. ${ }^{42}$ Due to the short life span of Drosophila, it is an ideal model organism to study development and aging related disorders at different life stages.

Our lab has developed methods to study stimulated dopamine and serotonin release in Drosophila larvae. Blue light, red light or ATP stimulated dopamine and serotonin release have been measured and characterized in transgenic Drosophila larva. Stimulated dopamine concentrations, rate of dopamine clearance and $K m$ values obtained in larval ventral nerve cord (VNC) are similar to electrically evoked release in mammals. ${ }^{43,44}$ Dopamine release and uptake parameters are different in different regions of the larval CNS. ${ }^{35}$ Synthesis and reuptake of both dopamine and serotonin are needed to maintain the releasable pool of the neurotransmitters, and the dynamics of release and clearance of both neurotransmitters are similar to mammals. ${ }^{39,45}$

Past research in our lab has established that Drosophila dopamine and serotonin neurotransmission are similar to mammals and that the model organism can be valuable in understanding the neurotransmitter systems better. However, we were not able to measure stimulated release in adult Drosophila. Chapter 3 of this thesis describes a method I have used to stimulated dopamine release in adult Drosophila. 


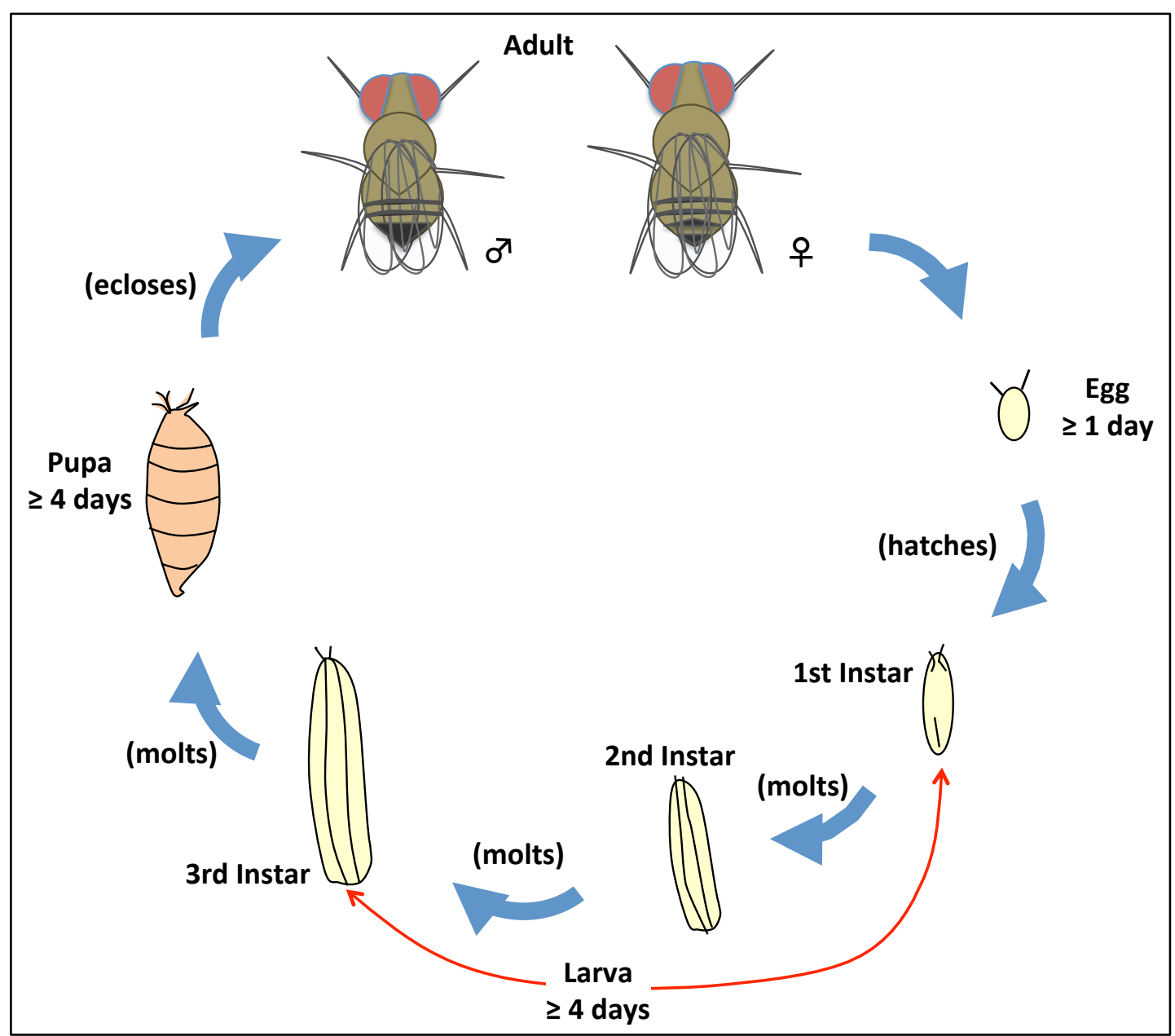

Figure 1.3. Life cycle of Drosophila melanogaster. The life cycle of Drosophila takes 9-10 days to complete at $25^{\circ} \mathrm{C}$. Embryo hatch from fertilized eggs in $\sim 1$ day. The larval stage lasts $\sim 4$ days where the larvae grow in the food feeding generously. The third instar larva crawls out of the food to a dry part of the vial. It changes color becoming darker as it pupates. After $\sim 4$ day in the pupal stage the adult fly ecloses. The life cycle takes longer to complete at colder temperatures.

\subsection{Neurotransmitters dopamine, octopamine and acetylcholine}

Drosophila and humans share many common neurotransmitters -

dopamine, serotonin, acetylcholine, glutamate and GABA. In addition to these, humans have epinephrine and norepinephrine, which are analogous to tyramine and octopamine in fruit flies (Fig. 1.4). Dopamine is a catecholamine neurotransmitter that plays a role in the reward pathways and in addiction. ${ }^{46}$ It is 
also involved in motor control ${ }^{47}$ and is the major neurotransmitter affected in Parkinson's disease ${ }^{48}$ Many aspects of dopamine dynamics and signaling are conserved between humans and Drosophila and many drugs have the same effects in the dopaminergic system in both mammals and Drosophila, making it an excellent model organism for drug screening. ${ }^{49}$ For example, tyrosine hydroxylase $(\mathrm{TH})$ and aromatic L-amino acid decarboxylase (DDC) are the synthesis precursors for dopamine (Fig. 1.5) in both humans and Drosophila, and dopamine is packaged into vesicles through vesicular monoamine transporters (VMAT). Dopamine transporters (DAT) reuptakes released dopamine back into the presynaptic cells in both humans and Drosophila.

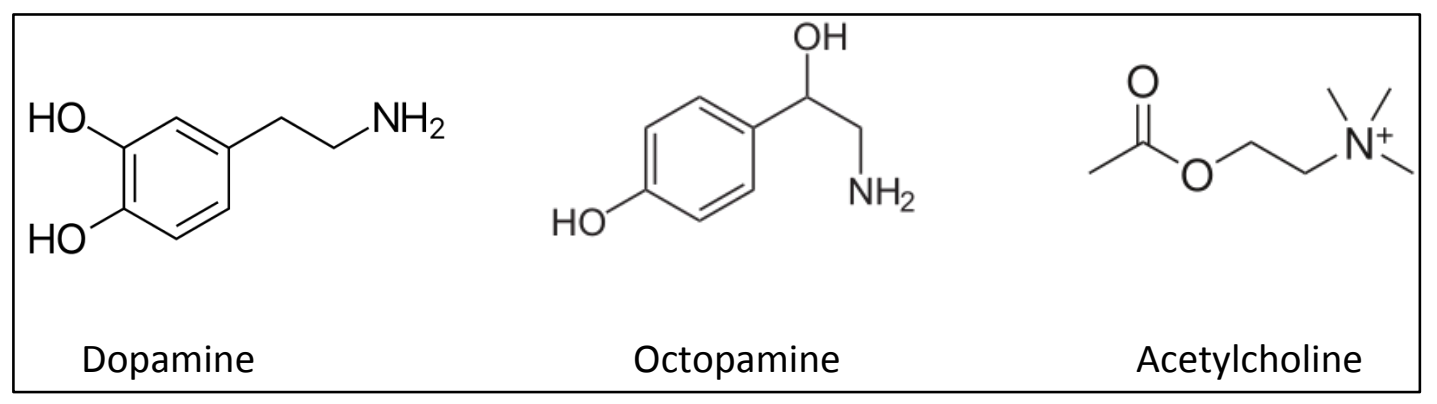

Figure 1.4. Chemical structures of neurotransmitters studied here.

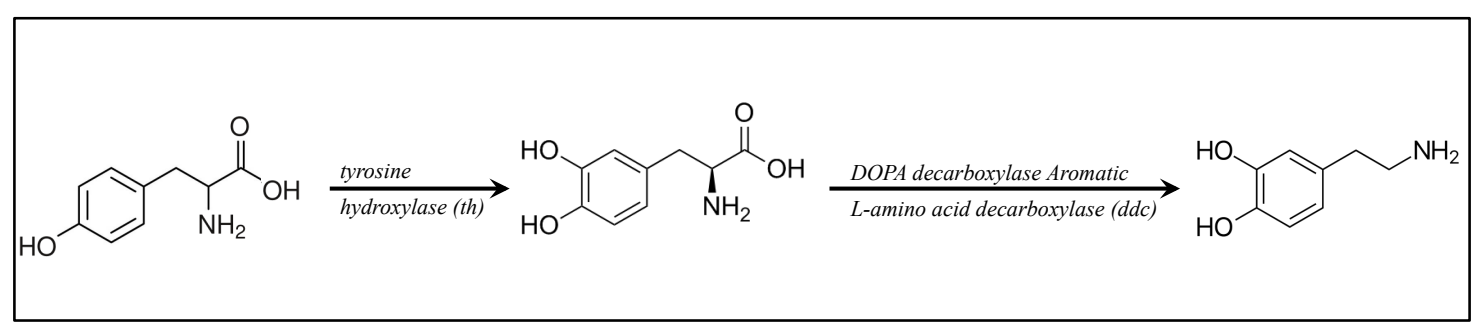

Figure 1.5. Synthesis pathway of dopamine. L-tyrosine is first converted to L-3,4dihydroxyphenylalanine (L-dopa) by the enzyme tyrosine hydroxylase. L-dopa is then converted to dopamine by DOPA decarboxylase (DDC). 
Due to the conserved structures between the two species, pharmacological agents have similar cellular effects in both humans and Drosophila. Studies of dopamine in Drosophila can identify potential drugs to be tested in the humans. ${ }^{50}$ Current therapy for Parkinson's disease is symptomatic, and does not treat the underlying cause of the disease or slow its progression. Screening tests in Drosophila model of Parkinson's disease has found that celastrol and minocycline, which have antioxidant and anti-inflammatory properties slows dopaminergic cell loss ${ }^{51}$ We have also found that the protein RNF11, which is downregulated in Parkinson's disease increases stimulated dopamine release and clearance in Drosophila suggesting a compensatory mechanism for the cell loss. ${ }^{52}$ Dopamine in Drosophila Parkinson's disease model has been further investigated as is described in Chapter 3 of this thesis.

Octopamine is a phenolamine that acts as a neurotransmitter, neurohormone, and neuromodulator in many invertebrates. It is analogous to norepinephrine in mammals and synthesized by homologous pathways. ${ }^{53} \mathrm{In}$ insects, the biosynthesis of octopamine is a two-step process where L-tyrosine is converted to tyramine by the enzyme tyrosine decarboxylase (TDC), and tyramine is converted to octopamine by tyramine beta hydroxylase (Tßh) (Fig. 1.6). There are two types of $T d c$ genes identified is Drosophila melanogaster, $T d c 1$, which is mostly expressed in non-neural tissues, and $T d c 2$, which is expressed in the CNS. Similar to dopamine, octopamine is packaged into 
vesicles by VMAT. Unlike dopamine, however, there are no octopamine transporters identified in Drosophila melanogaster.

In insects, octopamine is involved in many important functions such as ovulation ${ }^{54}$, olfactory learning and memory, ${ }^{55}$ aggression, ${ }^{56}$ and locomotion and grooming. ${ }^{57}$ In mammals, octopamine is found in very small amounts and is suggested to play a role in modulating other neurotransmitters. ${ }^{58}$ As it is a major invertebrate neurotransmitter and is suggested to play important roles in mammalian nervous system, I studied stimulated octopamine release in Drosophila larvae as described in Chapter 4.

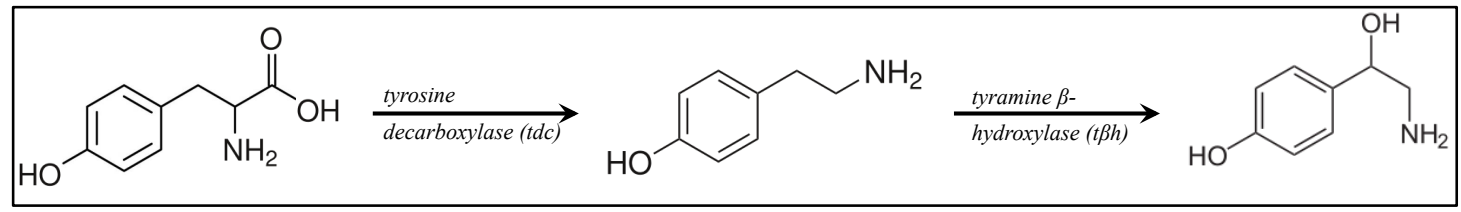

Figure 1.6. Octopamine synthesis pathway. L-tyrosine is first converted to tyramine by the enzyme tyrosine decarboxylase (tdc). Tyramine is then converted to octopamine by tyramine beta hydroxylase $(\mathrm{t} \beta \mathrm{h})$.

Acetylcholine is another major excitatory neurotransmitter in the CNS of insects. Cholinergic transmission is involved in mechanosensory responses, ${ }^{59}$ wing movements ${ }^{60}$ and in learning and memory formation. ${ }^{61}$ Many insecticides like organophosphates, carbamates and neonicotinoids exert their toxic activity by disrupting the normal function of the cholinergic system. There are two broad classes of receptors acetylcholine acts on - nicotinic acetylcholine receptor (nAChR) and muscarinic acetylcholine receptor (mAchR). Nicotinic receptor, which are sensitive to nicotine and neonicotinoids are more abundant than muscarinic type in the nervous system of insects. ${ }^{62,63} \mathrm{nAChR}$ activation causes 
dopamine release in mammals; however, there are no such reports in Drosophila. As described in Chapter 2 of this thesis, I have discovered and characterized nAhcR mediated dopamine release in Drosophila larva.

\subsubsection{Effect of insecticides on insect neurotransmitters}

As mentioned above, many insecticides act on the nervous system of insects. Cocaine ${ }^{64}$ and nicotine ${ }^{65}$ are natural insecticides that potentiate neurotransmission and cause hyperactivity, spasmodic event and akinesia. ${ }^{66}$ Responses to cocaine and nicotine is modulated by dopaminergic pathways in Drosophila. ${ }^{66}$ Nicotine interacts with nAChRs, which are pentamers made of homologous or heterologous subunits. ${ }^{67,68}$ Drosophila melanogaster nAChRs consist of ten different subunits (Da1 - Da7 and D $\beta 1-D \beta 3) .{ }^{69}$ Electrophysiological studies show fast transient synaptic currents mediated by nAChRs in cultured Drosophila embryonic neurons. ${ }^{70}$ Drosophila Kenyon cells also exhibit an increase in intercellular $\left[\mathrm{Ca}^{2+}\right]$ in response to $\mathrm{nAChR}$ stimulation. ${ }^{71}$

Neonicotinoids are synthetic analogs of nicotine with lower mammalian toxicity and longer field stability. Similar to nicotine, they increase nerve transmission by interacting with acetylcholine receptors and increasing intercellular $\left[\mathrm{Ca}^{2+}\right]{ }^{72}$ They are a widely used class of insecticide, and covered $80 \%$ of total the insecticidal seed treatment market in $2008 .{ }^{73}$ There are several types of neonicotinoids with different sensitivities towards different insects. ${ }^{73}$ Insects can develop resistance against certain neonicotinoids, which in some cases has been related to $\mathrm{nAChR}$ subunit mutation(s). A single point mutation in 
the $\beta 1$ gene was found in 5 field populations of neonicotinoid imidacloprid resistant Aphis gossypii. ${ }^{74}$ Mutations in the $\mathrm{nAChR}$ subunit genes in Drosophila melanogaster also created lines resistant to neonicotinoids. ${ }^{75}$ Resistance to neonicotinoids is a threat to the global agriculture market and target-site investigation of the neonicotinoids can aid in developing efficient resistant management strategies. ${ }^{73}$ Drosophila can be used to investigate $\mathrm{nAChR}$ subunit mutations that confer neonicotinoid resistance. This is particularly helpful since functional insect nAChRs are difficult to express in host cells.

\subsection{Dopamine in Parkinson's disease}

Parkinson's disease is a neurodegenerative disease that affects an estimated 10 million people in the world, and costs nearly $\$ 25$ billion per year to the United States. ${ }^{76,77}$ It is a debilitating condition that mostly affects the elderly population. The disease is due to a loss of dopaminergic cell bodies in the substantia nigra of the brain, and reduced dopamine levels. ${ }^{78-80}$ There is no treatment for Parkinson's disease, and medications that replace dopamine or are dopaminergic agonists only manage the symptoms. Major symptoms of Parkinson's disease are rest tremor, bradykinesia, rigidity and postural problems. ${ }^{81}$ Although medications manage the symptoms, patients usually start developing severe side effects after 5 years of being on medication. ${ }^{82}$ When Parkinson's disease is diagnosed, approximately $80 \%$ of the dopaminergic cells have already died. If the disease could be diagnosed earlier, the cell loss may be 
prevented. However, currently there is no method for early disease diagnosis. Parkinson's disease is a complex condition that affects many neurotransmitter systems. ${ }^{83-87}$ Therefore, it is imperative to understand how the different systems play a role in the disease. As genetic manipulations can be precisely controlled in Drosophila, it is an excellent model organism to study the cellular and molecular mechanisms of the disease to understand the cause and progression of the disease and to develop better medications.

There are several Drosophila lines that model Parkinson's disease. Effects of Parkinson's disease like mitochondria dysfunction, ${ }^{88}$ muscle weakness, ${ }^{89}$ and gait problems ${ }^{90}$ are similar to phenotypes seen in Drosophila with Parkinson's disease mutations. ${ }^{24,91} \mathrm{~A}$ protein, RNF11, is downregulated in Parkinson's disease patients, and we found that in Drosophila it plays a role in dopamine neurotransmission. ${ }^{52}$ In Drosophila that have a knockdown of the gene homologous to $R N F 11$, there is an increase in stimulated dopamine release and clearance. This study was done in larval Drosophila, and Chapter 3 of this thesis describes study of Parkinson's disease model Drosophila at different life stages.

\subsubsection{Parkinson disease model Drosophila melanogaster}

Mutations in the Parkin, ${ }^{92}$ PTEN-induced putative kinase 1 (PINK1), ${ }^{93}$ and alpha-synuclein ${ }^{94}$ genes have been linked to Parkinson's disease, but the mechanism of how the mutations lead to the disease is unclear. Recent work in Drosophila has provided evidence that both PINK1 and Parkin are involved in a pathway that regulates mitochondria dynamics ${ }^{95}$ and its ability to cope with 
oxidative stress. ${ }^{96}$ Mutant Drosophila have symptoms such as muscle loss ${ }^{24}$ and degeneration of dopaminergic neurons,${ }^{96}$ which are similar to humans with Parkinson's disease. ${ }^{89,97} \mathrm{~A}$ study in Drosophila also suggested a link between PINK1 and Parkin, which act on a linear pathway, and that Parkin functions downstream of PINK1. ${ }^{98}$ More work on the Parkinson's disease model fruit flies can provide further details on the mechanism of the disease. I have studied stimulated dopamine release in Drosophila with Parkin mutation as described in Chapter 3.

Drosophila melanogaster has several advantages as a model organism. It has already answered many fundamental questions about neurotransmission and neurological disorders. Using a rapid electrochemical technique, FSCV, I have studied the real time dynamics of neurotransmitters in Drosophila to better understand neurochemical changes during insecticide resistance and in neurological disorder, Parkinson's disease.

\subsection{Electrochemical detection of neurotransmitters}

\subsubsection{Introduction to electrochemistry}

Electrochemistry is a branch of chemistry that studies the relation between electrical and chemical changes. An electrochemical reaction occurs when an applied current causes a chemical reaction, or when a chemical reaction produces a current; this thesis deals with the latter process. A redox reaction is an electrochemical reaction where there is a transfer of electrons; the species that loses electrons is oxidized and the species that gains electrons is reduced. 
When a redox reaction occurs between neurotransmitters and the electrode, there is charge transfer at the interface of the electrode and the analyte. The charge is proportional to the number of moles of analyte reacted (Equation 1.1).

$$
\mathrm{Q}=\mathrm{nF} \Delta \mathrm{N}
$$

where $\mathrm{Q}=$ charge, $\mathrm{n}=$ number of electrons involved in the reaction, $\mathrm{F}=$ Faraday's constant and $\mathrm{N}=$ number of moles reacted.

Current is the rate of flow of charge (Equation 1.2), which can be measured and the number of moles of analyte calculated.

$$
i=\frac{d Q}{d t}
$$

where $\mathrm{i}=$ current, $\mathrm{Q}=$ charge and $\mathrm{t}=$ time

\subsubsection{Carbon fiber microelectrode for electrochemical detection}

Carbon fibers are 5-10 $\mu \mathrm{m}$ in diameter and are mostly composed of carbon. They have several advantages including high tensile strength, high stiffness and high chemical resistance. ${ }^{99,100}$ Carbon fiber electrodes were first used for the detection of neurotransmitters and their metabolites with pulse polarography. ${ }^{101}$ Since then, carbon fiber microelectrodes (CFMEs) have been a popular biosensor especially for the detection of neurotransmitters. ${ }^{45,} 102-105 \mathrm{~A}$ CFME is made of carbon fiber in insulated glass with protruding carbon length of $50-100 \mu \mathrm{m}$ and a diameter of $7 \mu \mathrm{m}$. The small size of the CFME makes it ideal for measurements in specific brain areas as well as in small preparations such as Drosophila CNS. Carbon fibers also have advantages of biocompatibility and well characterized electrochemical properties. ${ }^{102}$ Unmodified CFMEs have been used 
for the detection of several neurotransmitters - dopamine, serotonin, norepinephrine, epinephrine, octopamine, tyramine, histamine, and adenosine. ${ }^{45,}$ ${ }^{102-106}$ CFMEs can also be modified with enzymes to detect non-electroactive neurotransmitters like acetylcholine and glutamate. ${ }^{107,} 108$

\subsubsection{Electrochemical detection of neurotransmitters at CFMEs}

The main electrochemical methods used for measurements of

neurotransmitters at CFMEs are amperometry, ${ }^{109}$ chronoamperometry ${ }^{110}$ and fast-scan cyclic voltammetry (FSCV).${ }^{34}$ CFMEs are well suited for real time detection of the rapidly changing levels of neurotransmitters in the brain. In amperometry, a constant potential is applied to the electrode and neurotransmitters are oxidized as they come in contact with the electrode. The technique has high temporal resolution but is not selective, which makes it not ideal for use in complex biological samples. Choronoamperometry is a potential step method and measures the ratio of oxidation to reduction currents at a given potential. For accurate measurements, it is important to keep the time between the potential steps long enough to separate faradaic current from charging current. Also, a large charging current is generated if the potential step is too large, making it different to detect faradaic current. ${ }^{111} \mathrm{FSCV}$ is a potential sweep method where the voltage is ramped up and down, and current response measured at different potentials. A background current must be subtracted to obtain a voltammogram. The shape of the voltammogram helps with compound identification as it has compound specific characteristics and peak potentials. 
This chemical specificity gives FSCV a unique advantage over other electrochemical techniques. This thesis describes studies of neurotransmitters dopamine and octopamine with FSCV.

\subsection{FSCV data collection and analysis}

As mentioned in section 1.5.1, charge transfer at the interface of the electrode and analyte solution generates current, which is a Faradaic process. Any current generated by the movement of ions in solution without exchange of electrons at the interface is non-Faradaic (background current) and needs to be subtracted out from the Faradaic current. The main factors that increase the nonFaradaic charging current are increasing the electrode area or the scan rate. However, the charging current is stabilized after few minutes of waveform application, and can be subtracted out (background subtraction) to obtain just the Faradaic current. Before sample injection or stimulation in vivo, background current is collected for a few seconds.

Figure 1.7 describes FSCV detection of $1 \mu \mathrm{M}$ dopamine in vitro. Figure 1.7A shows the FSCV waveform used for dopamine detection. This waveform has been optimized for sensitive measurement of dopamine at CFMEs. ${ }^{112,113}$ Scanning at a high rate creates a large background current (Fig. 1.7B). The background current is stable and can be subtracted. Figure 1.7B shows the background currents with and without dopamine. A characteristic cyclic voltammogram (CV) (Fig. 1.7C) can be obtained after subtracting the background 
current, which for dopamine has the oxidation peak around $0.6 \mathrm{~V}$ and reduction peak around $-0.2 \mathrm{~V}$ versus $\mathrm{Ag} / \mathrm{AgCl}$ electrode. Dopamine oxidizes around $0.2 \mathrm{~V}$ and the observed shift in the peak is a result of the high scan rate used in FSCV, which delays the time between the reaction and its detection. A false color plot is generated as multiple CVs are collected over time (Fig. 1.7D). A horizontal section of the color plot shows how the current changes with time at a particular voltage. Figure 1.7C (inset) shows the current vs. time plot at $0.6 \mathrm{~V}$. Each electrode is calibrated in vitro and because current is linearly proportional to concentration, the concentration of neurotransmitters released upon stimulation in vivo can be calculated.

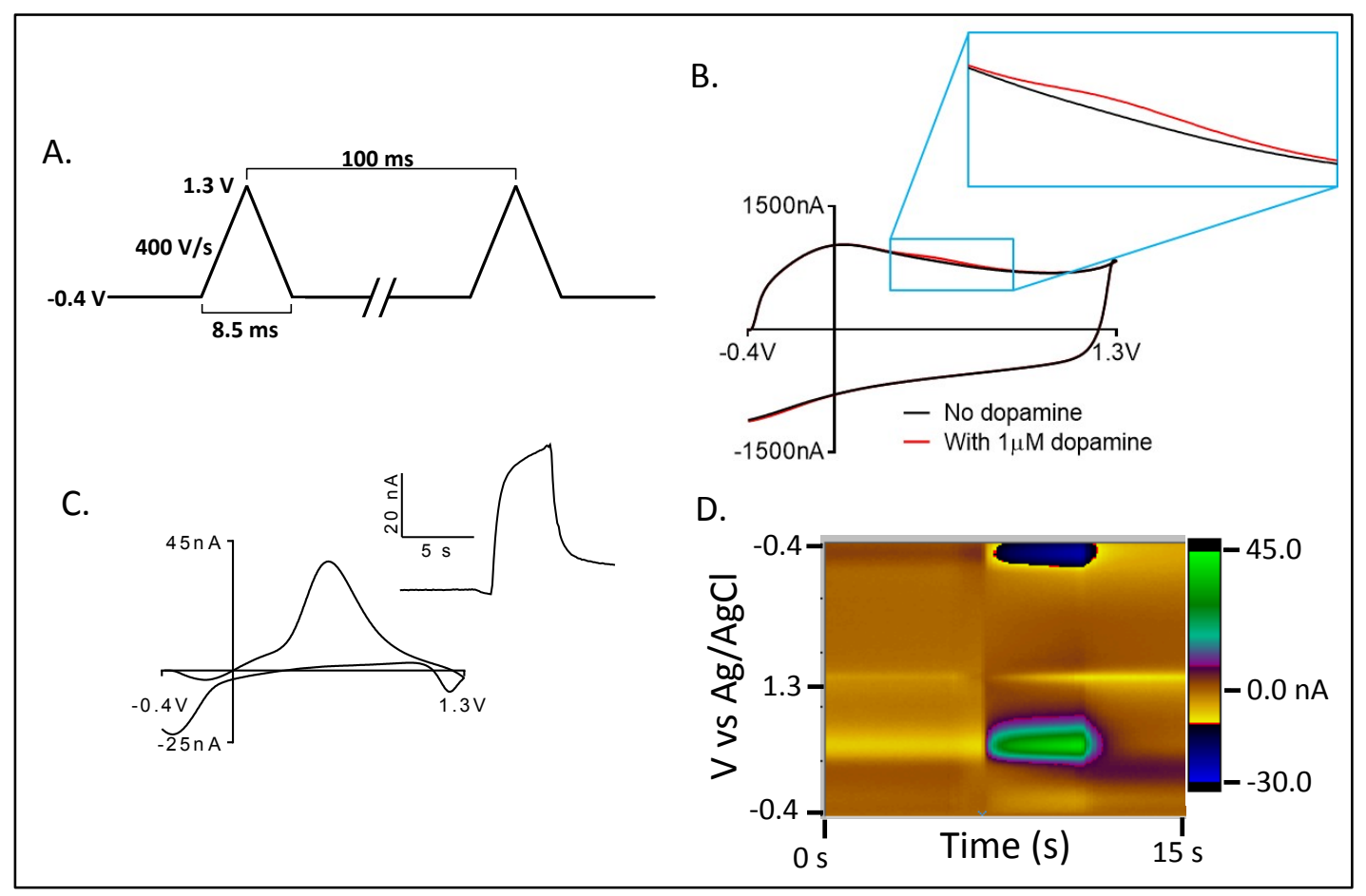


Figure 1.7. FSCV detection of dopamine in vitro. (A) FSCV waveform optimized for dopamine detection. (B) Scanning at a high rate creates a large background current (black line) which is stable. When dopamine is injected the current is slightly higher when dopamine oxidizes (red line). Since the background current is stable, it can be subtracted out to get the CV for dopamine. (C) Characteristic CV for dopamine with oxidation peak around $0.6 \mathrm{~V}$ and reduction peak around $-0.2 \mathrm{~V}$. The current vs. time plot at $0.6 \mathrm{~V}$ (inset). (D) False color plot with multiple background subtracted CVs. The color plot can be used to extract CVs at different times and current vs. time plots at different potentials. The large green oval is the time when dopamine was injected and oxidized. The blue oval at the top is the reduction of the dopamine oxidation product.

Properties of the electrode and measurement parameters affect the current as shown in Equation 1.3, which is for adsorption controlled redox reaction, and applies to all redox reactions described in this thesis.

$$
i_{p}=\frac{n^{2} F^{2} v A \Gamma^{*}}{4 R T}
$$

where $i_{p}=$ peak current, $n=$ number of electrons involved in the reaction, $F=$ Faraday's constant, $v=$ scan rate, $\mathrm{A}=$ electrode surface area, $\Gamma^{*}=$ moles of adsorbed reactant

\subsubsection{Redox mechanisms for dopamine and octopamine}

Dopamine oxidation is a two-electron process, where dopamine is

oxidized to dopamine-o-quinone (DOQ) that can reduce back to dopamine..$^{114}$

The conversion of dopamine to DOQ is a reversible process, and some DOQ is reduced back to dopamine. DOQ can also cyclize to leucidopaminochrome, which can again oxidize to dopaminochrome. However, the cyclization rate for dopamine is low compared to other catecholamines. ${ }^{115}$ Only the primary oxidation and reduction peaks are observed in the CV because of the slow cyclization and the low concentration of dopamine studied with FSCV.

Octopamine is a phenol with a different reaction mechanism. It is proposed that octopamine first goes through a one-step oxidation at the phenol 
creating a radical. ${ }^{104}$ The radical then reacts with another octopamine molecule to form a radical dimer. The dimer can further oxidize to create a product that can again react with more octopamine molecules to create a polymer. There are two oxidation peaks observed on octopamine CV, where the second peak lasts much longer than the first one. This long lasting secondary peak is thought to foul the electrode as subsequent measurements of octopamine with FSCV at standard dopamine waveform gave a decreasing response.

\subsubsection{FSCV waveform optimization}

The most popular waveform used with FSCV is triangular. Typical holding (lower) and switching (upper) potentials for dopamine measurements are $-0.4 \mathrm{~V}$ and 1.3 V, respectively (Fig. 1.7A). The rate at which the voltage is ramped, the scan rate, is typically $400 \mathrm{~V} / \mathrm{s}$, and the waveform application frequency is typically $10 \mathrm{~Hz}$ for dopamine measurement. The negative holding potential of $-0.4 \mathrm{~V}$ is kept for a relatively long time so the electrode can attract and adsorb the positively charged neurotransmitters. ${ }^{116}$ When the voltage is ramped up and reaches the analyte's oxidation potential, the neurotransmitter is oxidized and when the voltage is ramped back down, it is reduced. The high scan rate and frequency allow rapid measurements and tracking of real time changes in neurotransmitter levels with high temporal resolution.

FSCV waveforms are usually optimized for the detection of specific analytes, such as serotonin, ${ }^{117}$ adenosine,${ }^{118}$ tyramine and octopamine.${ }^{119}$ For example, the waveform used for octopamine detection in vitro was optimized to 
be held at $0.1 \mathrm{~V}$ and ramped up to $1.3 \mathrm{~V}$ at $600 \mathrm{~V} / \mathrm{s}$. Octopamine oxidation reaction creates secondary products that can foul the electrode, and at $0.1 \mathrm{~V}$ holding potential the secondary peak was not observed. At high potentials, the carbon surface starts to fracture, which increases the surface area of the electrode, increasing analyte adsorption and reducing the limit of detection. ${ }^{120}$ In vitro octopamine detection with $1.4 \mathrm{~V}$ switching potential gave a higher signal due to secondary oxidation product compared to the main product. Therefore, a switching potential of 1.3 was considered optimal. The current detected with FSCV increases with scan rate, and a rate of $600 \mathrm{~V} / \mathrm{s}$ was chosen for in vitro detection of octopamine. This waveform, however, was not suitable for in situ detection of octopamine as the higher scan rate shifted the oxidation peak toward the switching potential. When used in tissues, the CV peaks often shift to higher potentials due to slowed electron transfer compared to in vitro measurements, hence skewing the peaks if they are too close to the switching potential. ${ }^{121}$ Chapter 3 of this thesis describes optimization of the FSCV waveform for the detection of octopamine in situ. By using a waveform with a negative holding potential, a more positive switching potential and a slower scan rate, I could measure stable octopamine responses both in vitro and in situ.

Fast-scan cyclic voltammetry (FSCV) is a rapid, sensitive and selective technique that when used with carbon fiber microelectrodes (CFME) can provide high spatial resolution measurements of neurotransmitters. This thesis describes real time detection of neurotransmitters in Drosophila melanogaster central 
nervous system with FSCV. In Chapter 2, I describe the first measurements of nicotine and acetylcholine stimulated dopamine release in Drosophila larvae. Nicotinic acetylcholine receptors mediate this release and subunit mutations control the extent to which neonicotinoid insecticides also stimulate release. In Chapter 3, I discuss the first measurements of acetylcholine stimulated dopamine release in adult Drosophila, and the study of stimulated dopamine and dopamine tissue content in Parkinson's disease model Drosophila. In Chapter 4, I report FSCV waveform optimization for the detection of octopamine. I also describe the use of this waveform for the first measurements of light and ATP stimulated octopamine in Drosophila larva. 


\subsection{References}

[1] Taber, K. H., and Hurley, R. A. (2014) Volume Transmission in the Brain: Beyond the Synapse, J Neuropsych Clin N 26.

[2] Bartus, R. T., Dean, R. L., Beer, B., and Lippa, A. S. (1982) The Cholinergic Hypothesis of Geriatric Memory Dysfunction, Science 217, 408-417.

[3] Kohler, S., Cierpinsky, K., Kronenberg, G., and Adli, M. (2016) The serotonergic system in the neurobiology of depression: Relevance for novel antidepressants, J Psychopharmacol 30, 13-22.

[4] Lindstrom, M. B., Ryding, E., Ahnlide, J. A., Bosson, P., Traskman-Bendz, L., and Rosen, I. (2005) Impulsivity related to serotonin transporter measured by SPECT in suicide attempters., Nord J Psychiat 59, 414-415.

[5] Hornig, M. (1998) Addressing comorbidity in adults with attentiondeficit/hyperactivity disorder, J Clin Psychiat 59, 69-75.

[6] Fearnley, J. M., and Lees, A. J. (1991) Ageing and Parkinson's disease: substantia nigra regional selectivity, Brain : a journal of neurology 114 ( Pt 5), 2283-2301.

[7] Fuke, S., Suo, S., Takahashi, N., Koike, H., Sasagawa, N., and Ishiura, S. (2001) The VNTR polymorphism of the human dopamine transporter (DAT1) gene affects gene expression, The pharmacogenomics journal 1, 152-156.

[8] Cheon, K. A., Ryu, Y. H., Kim, J. W., and Cho, D. Y. (2005) The homozygosity for 10-repeat in allele at dopamine transporter gene and dopamine transporter density in Korean children with attention deficit hyperactivity disorder: relating to treatment response to methylphenidate, Eur Neuropsychopharm 15, 95-101.

[9] Brisch, R., Saniotis, A., Wolf, R., Bielau, H., Bernstein, H. G., Steiner, J., Bogerts, B., Braun, K., Jankowski, Z., Kumaratilake, J., Henneberg, M., and Gos, T. (2014) The role of dopamine in schizophrenia from a neurobiological and evolutionary perspective: old fashioned, but still in vogue, Frontiers in psychiatry 5, 47. 
[10] Bunin, M. A., and Wightman, R. M. (1998) Quantitative evaluation of 5hydroxytryptamine (serotonin) neuronal release and uptake: An investigation of extrasynaptic transmission, J Neurosci 18, 4854-4860.

[11] Wiedemann, D. J., Garris, P. A., Near, J. A., and Wightman, R. M. (1992) Effect of Chronic Haloperidol Treatment on Stimulated Synaptic Overflow of Dopamine in the Rat Striatum, J Pharmacol Exp Ther 261, 574-579.

[12] Thomas, C. G., Tian, H., and Diamond, J. S. (2011) The relative roles of diffusion and uptake in clearing synaptically released glutamate change during early postnatal development, $J$ Neurosci $31,4743-4754$.

[13] Scimemi, A., and Beato, M. (2009) Determining the Neurotransmitter Concentration Profile at Active Synapses, Mol Neurobiol 40, 289-306.

[14] Morgan, T. H. (1910) Sex Limited Inheritance in Drosophila, Science 32, 120-122.

[15] Salkoff, L., Baker, K., Butler, A., Covarrubias, M., Pak, M. D., and Wei, A. G. (1992) An Essential Set of K(+) Channels Conserved in Flies, Mice and Humans, Trends Neurosci 15, 161-166.

[16] Benzer, S. (1967) Behaavioral Mutant of Dorsophila Isolated by Countercurrent Distribution, Proc Natl Acad Sci U S A 58, 1112-1119.

[17] Bellen, H. J., Tong, C., and Tsuda, H. (2010) TIMELINE 100 years of Drosophila research and its impact on vertebrate neuroscience: a history lesson for the future, Nat Rev Neurosci 11, 514-+.

[18] Jeibmann, A., and Paulus, W. (2009) Drosophila melanogaster as a Model Organism of Brain Diseases, Int J Mol Sci 10, 407-440.

[19] Pandey, U. B., and Nichols, C. D. (2011) Human Disease Models in Drosophila melanogaster and the Role of the Fly in Therapeutic Drug Discovery, Pharmacol Rev 63, 411-436.

[20] Hendricks, J. C., Finn, S. M., Panckeri, K. A., Chavkin, J., Williams, J. A., Sehgal, A., and Pack, A. I. (2000) Rest in Drosophila is a sleep-like state, Neuron 25, 129-138.

[21] Shaw, P. J., Cirelli, C., Greenspan, R. J., and Tononi, G. (2000) Correlates of sleep and waking in Drosophila melanogaster, Science 287, 1834-1837. 
[22] Feany, M. B., and Bender, W. W. (2000) A Drosophila model of Parkinson's disease, Nature 404, 394-398.

[23] Cha, G. H., Kim, S., Park, J., Lee, E., Kim, M., Lee, S. B., Kim, J. M., Chung, J., and Cho, K. S. (2005) Parkin negatively regulates JNK pathway in the dopaminergic neurons of Drosophila, Proc Natl Acad Sci U S A 102, 10345-10350.

[24] Greene, J. C., Whitworth, A. J., Kuo, I., Andrews, L. A., Feany, M. B., and Pallanck, L. J. (2003) Mitochondrial pathology and apoptotic muscle degeneration in Drosophila parkin mutants, P Natl Acad Sci USA 100, 4078-4083.

[25] Zwarts, L., Versteven, M., and Callaerts, P. (2012) Genetics and neurobiology of aggression in Drosophila, Fly 6, 35-48.

[26] Venken, K. J. T., and Bellen, H. J. (2005) Emerging technologies for gene manipulation in Drosophila melanogaster (vol 6, pg 167, 2005), Nat Rev Genet 6, 340-340.

[27] Ohnishi, O. (1977) Spontaneous and ethyl methanesulfonate-induced mutations controlling viability in Drosophila melanogaster. III. Heterozygous effect of polygenic mutations, Genetics 87, 547-556.

[28] Cooley, L., Kelley, R., and Spradling, A. (1988) Insertional mutagenesis of the Drosophila genome with single P elements, Science 239, 1121-1128.

[29] Giordano, E., Rendina, R., Peluso, I., and Furia, M. (2002) RNAi triggered by symmetrically transcribed transgenes in Drosophila melanogaster, Genetics 160, 637-648.

[30] Brand, A. H., and Perrimon, N. (1993) Targeted Gene-Expression as a Means of Altering Cell Fates and Generating Dominant Phenotypes, Development 118, 401-415.

[31] Duffy, J. B. (2002) GAL4 system in Drosophila: A fly geneticist's Swiss army knife, Genesis 34, 1-15.

[32] Agrawal, N., Dasaradhi, P. V. N., Mohmmed, A., Malhotra, P., Bhatnagar, R. K., and Mukherjee, S. K. (2003) RNA interference: Biology, mechanism, and applications, Microbiol Mol Biol R 67, 657-+. 
[33] Privman, E., and Venton, B. J. (2015) Comparison of Dopamine Kinetics in the Larval Drosophila Ventral Nerve Cord and Protocerebrum with Improved Optogenetic Stimulation, Journal of neurochemistry.

[34] Vickrey, T. L., Condron, B., and Venton, B. J. (2009) Detection of endogenous dopamine changes in Drosophila melanogaster using fastscan cyclic voltammetry, Analytical chemistry 81, 9306-9313.

[35] Privman, E., and Venton, B. J. (2015) Comparison of dopamine kinetics in the larval Drosophila ventral nerve cord and protocerebrum with improved optogenetic stimulation, J Neurochem 135, 695-704.

[36] Klapoetke, N. C., Murata, Y., Kim, S. S., Pulver, S. R., Birdsey-Benson, A., Cho, Y. K., Morimoto, T. K., Chuong, A. S., Carpenter, E. J., Tian, Z., Wang, J., Xie, Y., Yan, Z., Zhang, Y., Chow, B. Y., Surek, B., Melkonian, M., Jayaraman, V., Constantine-Paton, M., Wong, G. K., and Boyden, E. S. (2014) Independent optical excitation of distinct neural populations, Nature methods 11, 338-346.

[37] Ding, S., and Sachs, F. (1999) Single channel properties of P2X2 purinoceptors, J Gen Physiol 113, 695-720.

[38] North, R. A. (2002) Molecular physiology of P2X receptors, Physiol Rev 82, 1013-1067.

[39] Xiao, N., and Venton, B. J. (2015) Characterization of dopamine releasable and reserve pools in Drosophila larvae using ATP/P2X(2)-mediated stimulation, J Neurochem 134, 445-454.

[40] Littleton, J. T., and Ganetzky, B. (2000) lon channels and synaptic organization: Analysis of the Drosophila genome, Neuron 26, 35-43.

[41] Fredieu, J. R., and Mahowald, A. P. (1989) Glial Interactions with Neurons during Drosophila Embryogenesis, Development 106, 739-\&.

[42] Denno, M. E., Privman, E., and Venton, B. J. (2015) Analysis of Neurotransmitter Tissue Content of Drosophila melanogaster in Different Life Stages, Acs Chemical Neuroscience 6, 117-123.

[43] Vickrey, T. L., Xiao, N., and Venton, B. J. (2013) Kinetics of the Dopamine Transporter in Drosophila Larva, Acs Chemical Neuroscience 4, 832-837. 
[44] Vickrey, T. L., Condron, B., and Venton, B. J. (2009) Detection of Endogenous Dopamine Changes in Drosophila melanogaster Using FastScan Cyclic Voltammetry, Anal Chem 81, 9306-9313.

[45] Borue, X., Cooper, S., Hirsh, J., Condron, B., and Venton, B. J. (2009) Quantitative evaluation of serotonin release and clearance in Drosophila, $J$ Neurosci Meth 179, 300-308.

[46] Koob, G. F., and Simon, E. J. (2009) The Neurobiology of Addiction: Where We Have Been and Where We Are Going, J Drug Issues 39, 115-132.

[47] Herbin, M., Simonis, C., Reveret, L., Hackert, R., Libourel, P. A., Eugene, D., Diaz, J., de Waele, C., and Vidal, P. P. (2016) Dopamine Modulates Motor Control in a Specific Plane Related to Support, Plos One 11.

[48] Triarhou, L. C. (2002) Dopamine and Parkinson's disease - Introduction, Adv Exp Med Biol 517, 1-14.

[49] Yamamoto, S., and Seto, E. S. (2014) Dopamine Dynamics and Signaling in Drosophila: An Overview of Genes, Drugs and Behavioral Paradigms, Exp Anim Tokyo 63, 107-119.

[50] Lawal, H. O., Terrell, A., Lam, H. A., Djapri, C., Jang, J., Hadi, R., Roberts, L., Shahi, V., Chou, M. T., Biedermann, T., Huang, B., Lawless, G. M., Maidment, N. T., and Krantz, D. E. (2014) Drosophila modifier screens to identify novel neuropsychiatric drugs including aminergic agents for the possible treatment of Parkinson's disease and depression, Mol Psychiatr $19,235-242$.

[51] Faust, K., Gehrke, S., Yang, Y. F., Yang, L. C., Beal, M. F., and Lu, B. W. (2009) Neuroprotective effects of compounds with antioxidant and antiinflammatory properties in a Drosophila model of Parkinson's disease, Bmc Neurosci 10.

[52] Champaloux, E. P. (2016) Dopamine Kinetics in a Drosophila Model of Parkinson Disease [dissertation], University of Virginia, Charlottesville.

[53] Wallace, B. G. (1976) The biosynthesis of octopamine--characterization of lobster tyramine beta-hydroxylase, Journal of neurochemistry $26,761-770$. 
[54] Monastirioti, M. (2003) Distinct octopamine cell population residing in the CNS abdominal ganglion controls ovulation in Drosophila melanogaster, Dev Biol 264, 38-49.

[55] Hammer, M., and Menzel, R. (1998) Multiple sites of associative odor learning as revealed by local brain microinjections of octopamine in honeybees, Learn Memory 5, 146-156.

[56] Zhou, C., Rao, Y., and Rao, Y. (2008) A subset of octopaminergic neurons are important for Drosophila aggression, Nat Neurosci 11, 1059-1067.

[57] Saraswati, S., Fox, L. E., Soll, D. R., and Wu, C. F. (2004) Tyramine and octopamine have opposite effects on the locomotion of Drosophila larvae, J Neurobiol 58, 425-441.

[58] Berry, M. D. (2004) Mammalian central nervous system trace amines. Pharmacologic amphetamines, physiologic neuromodulators, Journal of neurochemistry 90, 257-271.

[59] Peters, S., and Kreulen, D. L. (1986) Fast and Slow Synaptic Potentials Produced in a Mammalian Sympathetic-Ganglion by Colon Distension, $P$ Natl Acad Sci USA 83, 1941-1944.

[60] Heinrich, R., Hedwig, B., and Elsner, N. (1997) Cholinergic activation of stridulatory behaviour in the grasshopper Omocestus viridulus (L), J Exp Biol 200, 1327-1337.

[61] El Hassani, A. K., Dacher, M., Gary, V., Lambin, M., Gauthier, M., and Armengaud, C. (2008) Effects of sublethal doses of acetamiprid and thiamethoxam on the behavior of the honeybee (Apis mellifera), Arch Environ Con Tox 54, 653-661.

[62] Salvaterra, P. M., and Foders, R. M. (1979) [2-Alpha-Bungarotoxin-I-125 and [Quinuclidinylbenzilate-H-3 Binding in Central Nervous Systems of Different Species, J Neurochem 32, 1509-1517.

[63] Lummis, S. C., and Sattelle, D. B. (1985) Binding of N-[propionyl$3 \mathrm{H}$ ]propionylated alpha-bungarotoxin and L-[benzilic-4,4'-3H] quinuclidinyl benzilate to CNS extracts of the cockroach Periplaneta americana, Comp Biochem Physiol C 80, 75-83. 
[64] Nathanson, J. A., Hunnicutt, E. J., Kantham, L., and Scavone, C. (1993) Cocaine as a Naturally-Occurring Insecticide, P Natl Acad Sci USA 90, 9645-9648.

[65] Steppuhn, A., Gase, K., Krock, B., Halitschke, R., and Baldwin, I. T. (2004) Nicotine's defensive function in nature, Plos Biol 2, 1074-1080.

[66] Bainton, R. J., Tsai, L. T. Y., Singh, C. M., Moore, M. S., Neckameyer, W. S., and Heberlein, U. (2000) Dopamine modulates acute responses to cocaine, nicotine and ethanol in Drosophila, Curr Biol 10, 187-194.

[67] Gill, J. K., Savolainen, M., Young, G. T., Zwart, R., Sher, E., and Millar, N. S. (2011) Agonist activation of alpha 7 nicotinic acetylcholine receptors via an allosteric transmembrane site, P Natl Acad Sci USA 108, 5867-5872.

[68] Corringer, P. J., Le Novere, N., and Changeux, J. P. (2000) Nicotinic receptors at the amino acid level, Annu Rev Pharmacol 40, 431-458.

[69] Lansdell, S. J., Collins, T., Goodchild, J., and Millar, N. S. (2012) The Drosophila nicotinic acetylcholine receptor subunits Dalpha5 and Dalpha7 form functional homomeric and heteromeric ion channels, Bmc Neurosci $13,73$.

[70] Lee, D., and O'dowd, D. K. (1999) Fast excitatory synaptic transmission mediated by nicotinic acetylcholine receptors in Drosophila neurons, $J$ Neurosci 19, 5311-5321.

[71] Campusano, J. M., Su, H., Jiang, S. A., Sicaeros, B., and O'Dowd, D. K. (2007) nAChR-Mediated calcium responses and plasticity in Drosophila Kenyon cells, Dev Neurobiol 67, 1520-1532.

[72] Jepson, J. E., Brown, L. A., and Sattelle, D. B. (2006) The actions of the neonicotinoid imidacloprid on cholinergic neurons of Drosophila melanogaster, Invertebrate neuroscience : IN 6, 33-40.

[73] Jeschke, P., Nauen, R., Schindler, M., and Elbert, A. (2011) Overview of the Status and Global Strategy for Neonicotinoids, J Agr Food Chem 59, 2897-2908.

[74] Koo, H. N., An, J. J., Park, S. E., Kim, J. I., and Kim, G. H. (2014) Regional susceptibilities to 12 insecticides of melon and cotton aphid, Aphis 
gossypii (Hemiptera: Aphididae) and a point mutation associated with imidacloprid resistance, Crop Prot 55, 91-97.

[75] Perry, T., Heckel, D. G., McKenzie, J. A., and Batterham, P. (2008) Mutations in $\mathrm{D}$ alpha 1 or $\mathrm{D}$ beta 2 nicotinic acetylcholine receptor subunits can confer resistance to neonicotinoids in Drosophila melanogaster, Insect Biochem Molec 38, 520-528.

[76] Valente, A. X. C. N., das Neves, R. P., and Oliveira, P. J. (2012) Epigenetic engineering to reverse the Parkinson's expression state, Parkinsonism Relat D 18, 717-721.

[77] Bhimani, R. (2014) Understanding the Burden on Caregivers of People with Parkinson's: A Scoping Review of the Literature, Rehabil Res Pract 2014, 718527.

[78] Groger, A., Kolb, R., Schafer, R., and Klose, U. (2014) Dopamine reduction in the substantia nigra of Parkinson's disease patients confirmed by in vivo magnetic resonance spectroscopic imaging, Plos One 9, e84081.

[79] Gibb, W. R. (1991) Neuropathology of the substantia nigra, Eur Neurol 31 Suppl 1, 48-59.

[80] Forno, L. S. (1996) Neuropathology of Parkinson's disease, J Neuropath Exp Neur 55, 259-272.

[81] Jankovic, J. (2008) Parkinson's disease: clinical features and diagnosis, $J$ Neurol Neurosur Ps 79, 368-376.

[82] Jankovic, J. (2005) Motor fluctuations and dyskinesias in Parkinson's disease: clinical manifestations, Movement Disord 20, S11-S16.

[83] Pifl, C., Kish, S. J., and Hornykiewicz, O. (2012) Thalamic noradrenaline in Parkinson's disease: Deficits suggest role in motor and non-motor symptoms, Movement Disord 27, 1618-1624.

[84] Shin, E., Rogers, J. T., Devoto, P., Bjorklund, A., and Carta, M. (2014) Noradrenaline neuron degeneration contributes to motor impairments and development of L-DOPA-induced dyskinesia in a rat model of Parkinson's disease, Exp Neurol 257, 25-38. 
[85] Gotti, C., Moretti, M., Bohr, I., Ziabreva, I., Vailati, S., Longhi, R., Riganti, L., Gaimarri, A., McKeith, I. G., Perry, R. H., Aarsland, D., Larsen, J. P., Sher, E., Beattie, R., Clementi, F., and Court, J. A. (2006) Selective nicotinic acetylcholine receptor subunit deficits identified in Alzheimer's disease, Parkinson's disease and dementia with Lewy bodies by immunoprecipitation, Neurobiol Dis 23, 481-489.

[86] Martin-Ruiz, C. M., Piggot, M., Gotti, C., Lindstrom, J., Mendelow, A. D., Siddique, M. S., Perry, R. H., Perry, E. K., and Court, J. A. (2000) Alpha and beta nicotinic acetylcholine receptors subunits and synaptophysin in putamen from Parkinson's disease, Neuropharmacology 39, 2830-2839.

[87] Polotis, M., and Niccolini, F. (2015) Serotonin in Parkinson's disease, Behav Brain Res 277, 136-145.

[88] Winklhofer, K. F., and Haass, C. (2010) Mitochondrial dysfunction in Parkinson's disease, Bba-Mol Basis Dis 1802, 29-44.

[89] Cano-de-la-Cuerda, R., Perez-de-Heredia, M., Miangolarra-Page, J. C., Munoz-Hellin, E., and Fernandez-de-las-Penas, C. (2010) Is There Muscular Weakness in Parkinson's Disease?, Am J Phys Med Rehab 89, 70-76.

[90] Doherty, K. M., van de Warrenburg, B. P., Peralta, M. C., Silveira-Moriyama, L., Azulay, J. P., Gershanik, O. S., and Bloem, B. R. (2011) Postural deformities in Parkinson's disease, Lancet Neurol 10, 538-549.

[91] Whitworth, A. J. (2011) Drosophila Models of Parkinson's Disease, Adv Genet 73, 1-50.

[92] Kitada, T., Asakawa, S., Hattori, N., Matsumine, H., Yamamura, Y., Minoshima, S., Yokochi, M., Mizuno, Y., and Shimizu, N. (1998) Mutations in the parkin gene cause autosomal recessive juvenile parkinsonism, Nature 392, 605-608.

[93] Valente, E. M., Abou-Sleiman, P. M., Caputo, V., Muqit, M. M. K., Harvey, K., Gispert, S., Ali, Z., Del Turco, D., Bentivoglio, A. R., Healy, D. G., Albanese, A., Nussbaum, R., Gonzalez-Maldonaldo, R., Deller, T., Salvi, S., Cortelli, P., Gilks, W. P., Latchman, D. S., Harvey, R. J., Dallapiccola, B., Auburger, G., and Wood, N. W. (2004) Hereditary early-onset Parkinson's disease caused by mutations in PINK1, Science 304, 11581160. 
[94] Polymeropoulos, M. H., Lavedan, C., Leroy, E., Ide, S. E., Dehejia, A., Dutra, A., Pike, B., Root, H., Rubenstein, J., Boyer, R., Stenroos, E. S., Chandrasekharappa, S., Athanassiadou, A., Papapetropoulos, T., Johnson, W. G., Lazzarini, A. M., Duvoisin, R. C., Dilorio, G., Golbe, L. I., and Nussbaum, R. L. (1997) Mutation in the alpha-synuclein gene identified in families with Parkinson's disease, Science 276, 2045-2047.

[95] Deng, H. S., Dodson, M. W., Huang, H. X., and Guo, M. (2008) The Parkinson's disease genes pink1 and parkin promote mitochondrial fission and/or inhibit fusion in Drosophila, P Natl Acad Sci USA 105, 1450314508.

[96] Whitworth, A. J., Theodore, D. A., Greene, J. C., Benes, H., Wes, P. D., and Pallanck, L. J. (2005) Increased glutathione S-transferase activity rescues dopaminergic neuron loss in a Drosophila model of Parkinson's disease, $P$ Natl Acad Sci USA 102, 8024-8029.

[97] Javoy-Agid, F., Hirsch, E. C., Dumas, S., Duyckaerts, C., Mallet, J., and Agid, Y. (1990) Decreased tyrosine hydroxylase messenger RNA in the surviving dopamine neurons of the substantia nigra in Parkinson's disease: an in situ hybridization study, Neuroscience 38, 245-253.

[98] Clark, I. E., Dodson, M. W., Jiang, C. G., Cao, J. H., Huh, J. R., Seol, J. H., Yoo, S. J., Hay, B. A., and Guo, M. (2006) Drosophila pink1 is required for mitochondrial function and interacts genetically with parkin, Nature 441, 1162-1166.

[99] Williams, W. S., Steffens, D. A., and Bacon, R. (1970) Bending Behavior and Tensile Strength of Carbon Fibers, J Appl Phys 41, 4893-\&.

[100] Noisternig, J. F. (2000) Carbon fibre composites as stay cables for bridges, Appl Compos Mater 7, 139-150.

[101] Ponchon, J. L., Cespuglio, R., Gonon, F., Jouvet, M., and Pujol, J. F. (1979) Normal pulse polarography with carbon fiber electrodes for in vitro and in vivo determination of catecholamines, Analytical chemistry 51, 1483-1486.

[102] Huffman, M. L., and Venton, B. J. (2009) Carbon-fiber microelectrodes for in vivo applications, Analyst 134, 18-24.

[103] Pihel, K., Walker, Q. D., and Wightman, R. M. (1996) Overoxidized polypyrrole-coated carbon fiber microelectrodes for dopamine 
measurements with fast-scan cyclic voltammetry, Analytical chemistry 68, 2084-2089.

[104] Cooper, S. E., and Venton, B. J. (2009) Fast-scan cyclic voltammetry for the detection of tyramine and octopamine, Anal Bioanal Chem 394, 329336.

[105] Swamy, B. E. K., and Venton, B. J. (2007) Subsecond detection of physiological adenosine concentrations using fast-scan cyclic voltammetry, Analytical chemistry 79, 744-750.

[106] Pihel, K., Schroeder, T. J., and Wightman, R. M. (1994) Rapid and Selective Cyclic Voltammetric Measurements of Epinephrine and Norepinephrine as a Method to Measure Secretion from Single Bovine Adrenal-Medullary Cells, Analytical chemistry 66, 4532-4537.

[107] Oldenziel, W. H., and Westerink, B. H. C. (2005) Improving glutamate microsensors by optimizing the composition of the redox hydrogel, Analytical chemistry 77, 5520-5528.

[108] Navera, E. N., Suzuki, M., Tamiya, E., Takeuchi, T., and Karube, I. (1993) Nafion-Coated Carbon-Fiber for Acetylcholine and Choline Sensors, Electroanal 5, 17-22.

[109] Majdi, S., Berglund, E. C., Dunevall, J., Oleinick, A. I., Amatore, C., Krantz, D. E., and Ewing, A. G. (2015) Electrochemical Measurements of Optogenetically Stimulated Quantal Amine Release from Single Nerve Cell Varicosities in Drosophila Larvae, Angewandte Chemie.

[110] Fuenzalida-Uribe, N., Meza, R. C., Hoffmann, H. A., Varas, R., and Campusano, J. M. (2013) nAChR-induced octopamine release mediates the effect of nicotine on a startle response in Drosophila melanogaster, Journal of neurochemistry 125, 281-290.

[111] Eckermann, A. L., Feld, D. J., Shaw, J. A., and Meade, T. J. (2010) Electrochemistry of redox-active self-assembled monolayers, Coordin Chem Rev 254, 1769-1802.

[112] Heien, M. L. A. V., Phillips, P. E. M., Stuber, G. D., Seipel, A. T., and Wightman, R. M. (2003) Overoxidation of carbon-fiber microelectrodes enhances dopamine adsorption and increases sensitivity, Analyst 128, 1413-1419. 
[113] Zachek, M. K., Park, J., Takmakov, P., Wightman, R. M., and McCarty, G. S. (2010) Microfabricated FSCV-compatible microelectrode array for realtime monitoring of heterogeneous dopamine release, Analyst 135, 15561563.

[114] Ke, N. J., Lu, S. S., and Cheng, S. H. (2006) A strategy for the determination of dopamine at a bare glassy carbon electrode: $p$ -

Phenylenediamine as a nucleophile, Electrochem Commun 8, 1514-1520.

[115] Hawley, M. D., Tatawawadi, S. V., Piekarski, S., and Adams, R. N. (1967) Electrochemical studies of the oxidation pathways of catecholamines, $J$ Am Chem Soc 89, 447-450.

[116] Kawagoe, K. T., Zimmerman, J. B., and Wightman, R. M. (1993) Principles of Voltammetry and Microelectrode Surface-States, J Neurosci Meth 48, 225-240.

[117] Jackson, B. P., Dietz, S. M., and Wightman, R. M. (1995) Fast-Scan Cyclic Voltammetry of 5-Hydroxytryptamine, Analytical Chemistry 67, 1115-1120.

[118] Ross, A. E., and Venton, B. J. (2014) Sawhorse Waveform Voltammetry for Selective Detection of Adenosine, ATP, and Hydrogen Peroxide, Analytical Chemistry 86, 7486-7493.

[119] Cooper, S. E., and Venton, B. J. (2009) Fast-scan cyclic voltammetry for the detection of tyramine and octopamine, Analytical and bioanalytical chemistry 394, 329-336.

[120] Hafizi, S., Kruk, Z. L., and Stamford, J. A. (1990) Fast Cyclic Voltammetry Improved Sensitivity to Dopamine with Extended Oxidation Scan Limits, $J$ Neurosci Meth 33, 41-49.

[121] Amatore, C., Saveant, J. M., and Tessier, D. (1983) Charge-Transfer at Partially Blocked Surfaces - a Model for the Case of Microscopic Active and Inactive Sites, J Electroanal Chem 147, 39-51. 
Chapter 2: Nicotinic acetylcholine receptor (nAChR) mediated dopamine release in larval Drosophila melanogaster 


\section{Chapter 2: Nicotinic acetylcholine receptor (nAChR) mediated dopamine release in larval Drosophila melanogaster}

\subsection{Abstract}

Acetylcholine is an excitatory neurotransmitter in the central nervous system of insects and the nicotinic acetylcholine receptor ( $\mathrm{nAChR}$ ) is a target for neonicotinoid insecticides. Functional insect $\mathrm{nAChRs}$ are difficult to express in host cells, and hence difficult to study. In mammals, acetylcholine and nicotine evoke dopamine release, but the extent to which this mechanism is conserved in insects was unknown. In intact larval ventral nerve cords (VNCs), we studied dopamine evoked by acetylcholine, nicotine, or neonicotinoids. Using fast-scan cyclic voltammetry, we confirmed dopamine was measured by its cyclic voltammogram and also by feeding Drosophila the synthesis inhibitor, 3iodotyrosine, which lowered the evoked dopamine response. Acetylcholine (1.8 pmol) evoked on average $0.43+/-0.04 \mu \mathrm{M}$ dopamine. Dopamine release significantly decreased after incubation with a-bungarotoxin, demonstrating the release is mediated by $\mathrm{nAChR}$, but atropine, a muscarinic $A C h R$ antagonist, had no effect. Nicotine $\left(t_{1 / 2}=71 \mathrm{~s}\right)$ and the neonicotinoids nitenpyram and imidacloprid ( $t_{1 / 2}=86 \mathrm{~s}, 121 \mathrm{~s}$ respectively) also evoked dopamine release, which lasted longer than acetylcholine-stimulated release $\left(t_{1 / 2}=19 \mathrm{~s}\right)$. Nicotinestimulated dopamine was significantly lower in the presence of sodium channel blocker, tetrodotoxin, showing that the agonist acts on presynaptic nAChRs. Drosophila that have mutations in the $\mathrm{nAChR}$ subunit a1 or $\beta 2$ have significantly lower neonicotinoid-stimulated release but no changes in nicotine-stimulated 
release. This work demonstrates that $\mathrm{nAChR}$ agonists mediate dopamine release in Drosophila larval VNC and that mutations in $\mathrm{nAChR}$ subunits affect how insecticides stimulate dopamine release.

\subsection{Introduction}

Acetylcholine is a neurotransmitter in the central nervous system (CNS) of insects and the neuromuscular junction in vertebrates. It is the most abundant neurotransmitter in the CNS of insects, ${ }^{1}$ where the cholinergic system mediates wing movements, ${ }^{2}$ locomotion, learning, and memory. ${ }^{3}$ The cholinergic system is also a target of insecticides such as organophosphates, ${ }^{4}$ that inactivate acetylcholinesterase, and neonicotinoids, ${ }^{5}$ that are acetylcholine receptor agonists. ${ }^{6}$ Neonicotinoid insecticides work by overstimulating nicotinic acetylcholine receptor (nAChRs), which causes hyperexcitation, paralysis, and death of insects. ${ }^{7}$ Acetylcholine receptors are either nicotine sensitive, muscarine sensitive (muscarinic acetylcholine receptor, mAchR), or of mixed nicotinic/muscarinic nature. ${ }^{8} \mathrm{nAChRs}$ are more abundant than mAChRs in the nervous system of insects, including Drosophila melanogaster. ${ }^{9-11}$ Acetylcholine modulates neural activity in insects. For example, it causes depolarization, mediated by nAChRs, in cockroach giant interneurons ${ }^{12}$ and elicits large excitatory currents and action potential bursts in the Drosophila larval central nervous system. ${ }^{13}$ In rodents, nicotine increases dopamine release during phasic activity ${ }^{14}$ and depletion of acetylcholine or the presence of an nAChR antagonist 
decreases stimulated dopamine.${ }^{15}$ However, there are no studies of nAChR mediated dopamine release in insects.

Nicotinic acetylcholine receptors consist of five subunits that form a cation channel that is activated by acetylcholine interacting at the interface of two adjacent subunits. ${ }^{16,17}$ Benke and Breer were the first to suggest the existence of $\mathrm{nAChRs}$ in insects with differing affinity for a-bungarotoxin and different agonist sensitivities. ${ }^{18}$ Since then, several different types of nAChRs subunit combinations have been identified which have different agonist affinity and activation kinetics. ${ }^{19}$ Ten different subunits of nAChRs have been identified in Drosophila, Da1 - Da7 and D $\beta 1-D \beta 3^{20}$. a subunits contain a Cys-Cys pair, and are required for ligand binding. ${ }^{21}$ Small changes in nAChR subunits affect neonicotinoid sensitivity, as a single point mutation in the $\beta 1$ gene was identified in five field populations of Aphis gossypii resistant to the neonicotinoid imidacloprid. ${ }^{22}$ While not a plant pest, Drosophila melanogaster is a popular model organism to study the insect nervous system and is particularly helpful to study structures such as insect nAChRs that are difficult to express in host cells. ${ }^{23}$ Drosophila strains with mutations of Da1, Da2, or DB2 nAChR subunits are highly resistant to the neonicotinoids nitenpyram and imidacloprid, and mutations of different genes alter the resistance level. ${ }^{24}$ Studies in Drosophila have revealed that the a5 subunit is involved in a-bungarotoxin sensitivity of $\mathrm{nAChRs},{ }^{25}$ and that a6 subunit is essential for the insecticidal effect of spinosad. ${ }^{26}$ The behavioral effects of nicotine on Drosophila are modulated by 
dopamine,${ }^{27}$ so understanding how $\mathrm{nAChR}$ control dopamine release is critical for understanding the effects of neonicotinoids.

In this study, we characterized acetylcholine, nicotine, and neonicotinoidstimulated dopamine release in Drosophila larval ventral nerve cord (VNC) for the first time. Our lab has pioneered measurements of dopamine in Drosophila using fast-scan cyclic voltammetry (FSCV) at implanted carbon-fiber microelectrodes (CFMEs), but previous experiments mainly used optogenetically-stimulated release ${ }^{28,29}$ Here, we focus on release mediated by nAChRs and establish that acetylcholine, nicotine, and neonicotinoids cause dopamine release in the larval VNC. Longer duration release is evoked by nicotine and neonicotinoids than with acetylcholine. Stimulated release is sensitive to a-bungarotoxin (a-BTX) and tetrodotoxin (TTX) and is mediated by presynaptic nAChRs. Neonicotinoidstimulated release is significantly lower in Drosophila nAChR subunit mutants that were previously found to have increased resistance to imidacloprid and nitenpyram. Thus, mutations that confer resistance to neonicotinoids in these strains also affect neonicotinoid-stimulated dopamine release. nAChR agonists stimulate dopamine release in Drosophila larval VNC; therefore, Drosophila can be used to study agonist sensitivities at mutated nAChRs subunits. 


\subsection{Results}

\subsubsection{Acetylcholine stimulates dopamine release in Drosophila melanogaster larval VNC}

The effect of acetylcholine stimulation was studied in Canton S Drosophila melanogaster by pressure injection of acetylcholine into the neuropil of isolated larval VNC. A CFME and a pipet filled with $1 \mathrm{mM}$ acetylcholine were placed about $10 \mu \mathrm{m}$ apart (Fig. 2.1A); acetylcholine (1.8 nL droplet, or $1.8 \mathrm{pmol}$ ) was puffed into the larval VNC and the current response was measured with FSCV at the CFME. A trace of the concentration vs. time at the peak of oxidative potential (Fig. 2.1B) shows evoked dopamine changes over time while a trace of current vs. voltage, the cyclic voltammogram (inset), is a fingerprint of the molecule being detected. The cyclic voltammogram profile resembles that of dopamine with an oxidation peak around $0.6 \mathrm{~V}$ and reduction peak around $-0.2 \mathrm{~V}$. 


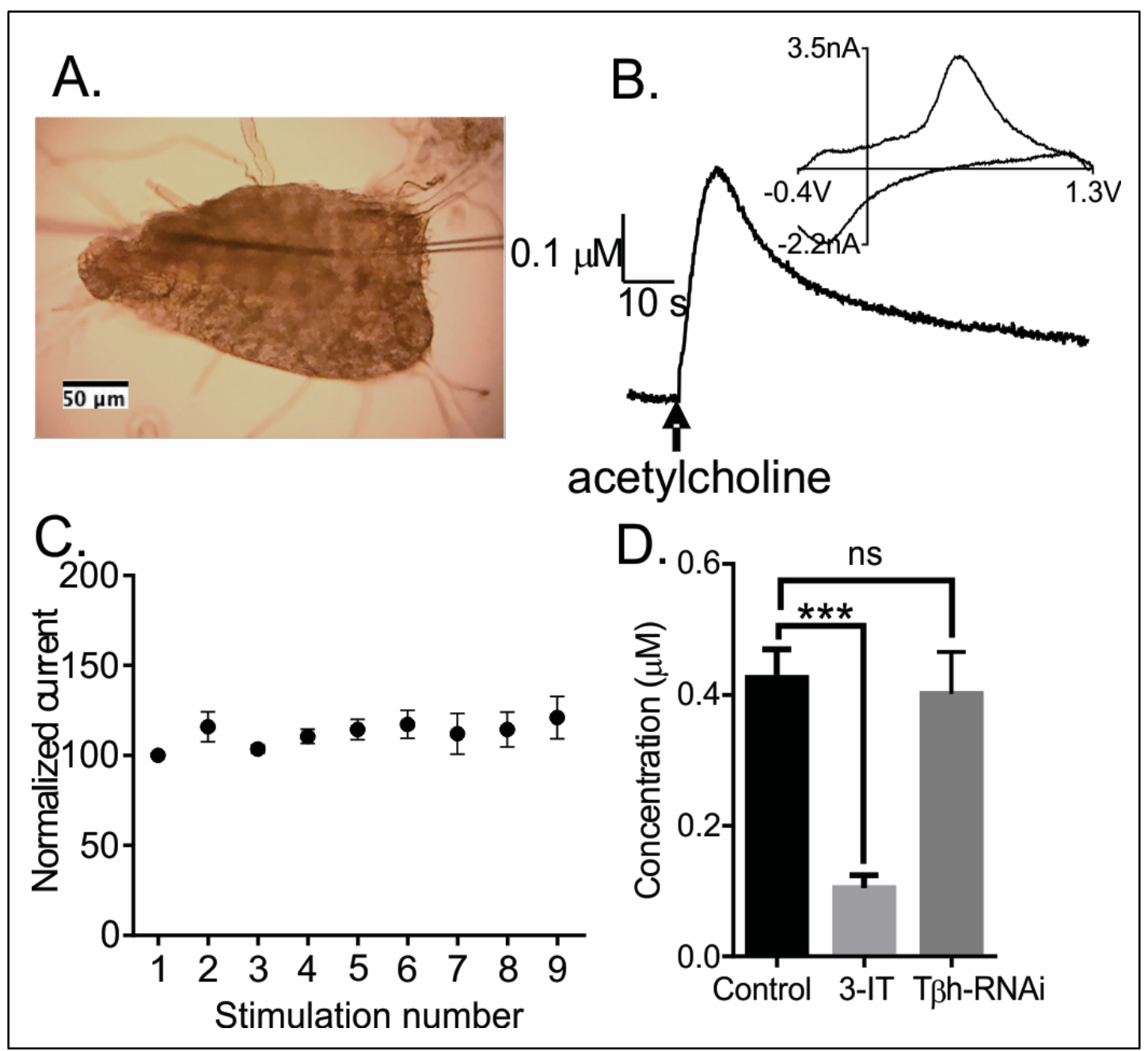

Figure 2.1. Acetylcholine-stimulated dopamine release. in Drosophila melanogaster ventral nerve cord (VNC). (A) Image showing electrode and stimulating pipet placed in VNC. (B) Acetylcholine (1.8 pmol) stimulated dopamine release measured with FSCV. The concentration vs time trace below shows changes in dopamine over time. The cyclic voltammogram confirms dopamine is detected. (C) When $1.8 \mathrm{pmol}$ acetylcholine stimulations are repeated at five minute intervals, the current response is stable (no effect of stimulation, one-way ANOVA, $p=0.66, n=6$ ). (D) Acetylcholine stimulated dopamine release in larvae fed with dopamine synthesis inhibitor, 3-iodotyrosine, is significantly lower than control (unpaired t -test, $p=0.0001, n=8-13$ ), which verifies that the response is due to dopamine. Current response in larvae with octopamine synthesis enzyme knockdown (Tdc2-GAL4;UAS-T $\beta h^{R N A j}$ ) is not significantly different than control (unpaired t-test, $p=$ $0.5678, n=5-13$ ) suggesting that the response is not due to octopamine.

To test the stability of acetylcholine mediated release, repeated measurements were taken in the same VNC (Fig. 2.1C). When $1.8 \mathrm{pmol}$ acetylcholine stimulation was repeated at 5 min intervals, the response was 
stable for 9 stimulations as there was no significant difference in the current response measured over subsequent stimulations (one-way ANOVA, $p=0.66, \mathrm{n}$ $=6)$.

To further verify that the response is due to dopamine release, larvae were fed with a dopamine synthesis inhibitor, 3-iodotyrosine (3-IT, $10 \mathrm{mg} / \mathrm{mL}$ in food for 48 hours). Acetylcholine evoked dopamine release $(0.43+/-0.04 \mu \mathrm{M})$ was significantly lower in larvae fed 3-IT $(0.10+/-0.02 \mu \mathrm{M}$, Fig. 2.1D) compared to control (unpaired t-test, $p=0.0001, \mathrm{n}=8-13$ ). Acetylcholine-stimulated response was not significantly different in larvae with knockdown of octopamine synthesis using RNAi, Tdc2-GAL4; UAS-RNAi ${ }^{T B H}$ (unpaired t-test, $p=0.5678, \mathrm{n}=5-13$ ), which proves that the response is not due to octopamine.

\subsubsection{Acetylcholine-stimulated release is mediated by $n A C h R$ and not mAchR}

There are two types of acetylcholine receptors $-\mathrm{nAChRs}$ that are activated by nicotine and mAchRs that are activated by muscarine. To test the effect of nAChRs on acetylcholine-stimulated release, nAChRs were blocked by a-BTX. Figure 2.2A and C show that in the presence of $2 \mu \mathrm{M}$ a-BTX $\left(K_{d}=0.008\right.$ and $1.14 \mathrm{nM}$ for high and low binding sites on aphid membrane $)^{30,31}$ the current is significantly lower than before the drug was applied (paired t-test, $p=0.0002, \mathrm{n}$ $=8)$. To test the effect of mAchRs on acetylcholine-stimulated release, mAchRs were blocked by atropine. Figure 2.2B and $\mathrm{C}$ show that in the presence of $1 \mu \mathrm{M}$ atropine $^{32}$ the current response is not significantly different before and after drug 
application (paired t-test, $p=0.6247, \mathrm{n}=5$ ). The results verify that acetylcholinestimulated dopamine release is mediated by nAChRs, and not mAchRs, in Drosophila larval VNC. In Drosophila, there are two different subtypes of nAChR, a-BTX sensitive or insensitive, ${ }^{23}$ so the lack of complete blockade by a-BTX suggests there could be a-BTX insensitive nAChRs in the larval VNC as well. 


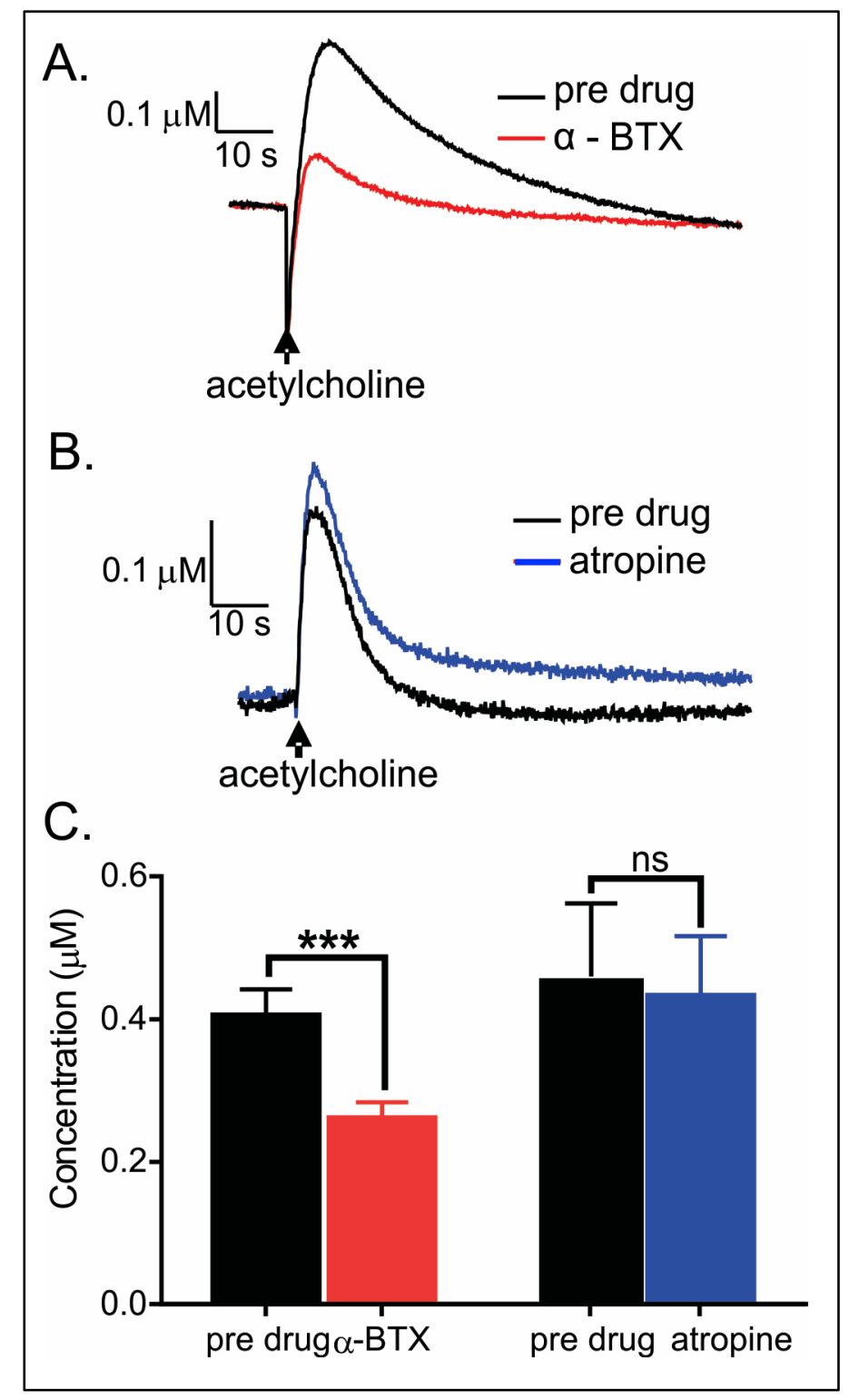

Figure 2.2. Acetylcholine-stimulated dopamine release is mediated by $n A C h R$ and not $m A c h R$. (A) Example data of acetylcholine-stimulated dopamine release before and after bathing in $\mathrm{nAChR}$ inhibitor, $2 \mu \mathrm{M}$ a-BTX. (B) Example data of acetylcholine-stimulated dopamine release before and after $\mathrm{mAchR}$ inhibitor, $1 \mu \mathrm{M}$ atropine. (C) Averaged data for the drugs. The current response is significantly lower after $a-B T X$ (paired $t$-test, $p=0.0002, n=8$ ) suggesting that the dopamine release is mediated by $n A C h R s$, while there is no significant change after atropine (paired $t$-test, $p=$ $0.6247, n=5)$, which shows that the dopamine release is not mediated by $\mathrm{mAch}$ s 


\subsubsection{Nicotine-stimulated dopamine release in Drosophila melanogaster larval VNC}

Nicotine is a natural insecticide that competes with acetylcholine to act at $\mathrm{nAChRs}$ and disrupt their normal function. A nicotine puff (1.8 or $8.8 \mathrm{fmol}$ ) in the VNC resulted in dopamine release, as verified by the CV (Fig. 2.3A-B). A lower amount of nicotine was used than acetylcholine because nicotine has a higher binding affinity $\left(K_{D}=15 n M\right)$ than acetylcholine $\left(K_{D}=180 \mathrm{nM}\right.$ in rat brain membranes) and would be less tightly regulated, as it is not a natural neurotransmitter. ${ }^{33,34}$ The $t_{1 / 2}$ value, which is the time it takes to go from the peak current to half the current, is significantly higher for nicotine-stimulated release $\left(t_{1 / 2}=71 \pm 5 \mathrm{~s}\right.$ for the $8.8 \mathrm{fmol}$ stimulation) than for acetylcholine-stimulated release ( $\mathrm{t}_{1 / 2}=19 \pm 5 \mathrm{~s} 1.8 \mathrm{pmol}$ stimulation) (one-way ANOVA multiple comparisons test, $p<0.05, \mathrm{n}=7$ ). Similar to acetylcholine-stimulated release, the response was significantly lower in larvae fed 3-IT, verifying that it is due to dopamine (unpaired t-test, $p=0.0345, \mathrm{n}=5-6$ ) (Fig. 2.3C). The nicotinestimulated response was not significantly different in flies with knockdown of octopamine synthesis, Tdc2-GAL4; UAS-RNAi ${ }^{T \beta H}$ (unpaired t-test, $p=0.3250, \mathrm{n}$ =4) (Fig. 2.3D). The sodium channel inhibitor TTX was used to examine whether release was exocytotic (Fig. 2.3E). The nicotine-stimulated response was significantly lower in the presence of TTX (paired t-test, $p=0.0007, \mathrm{n}=7$ ). Blocking sodium channels inhibits the firing of action potentials, and therefore also inhibits exocytosis. 


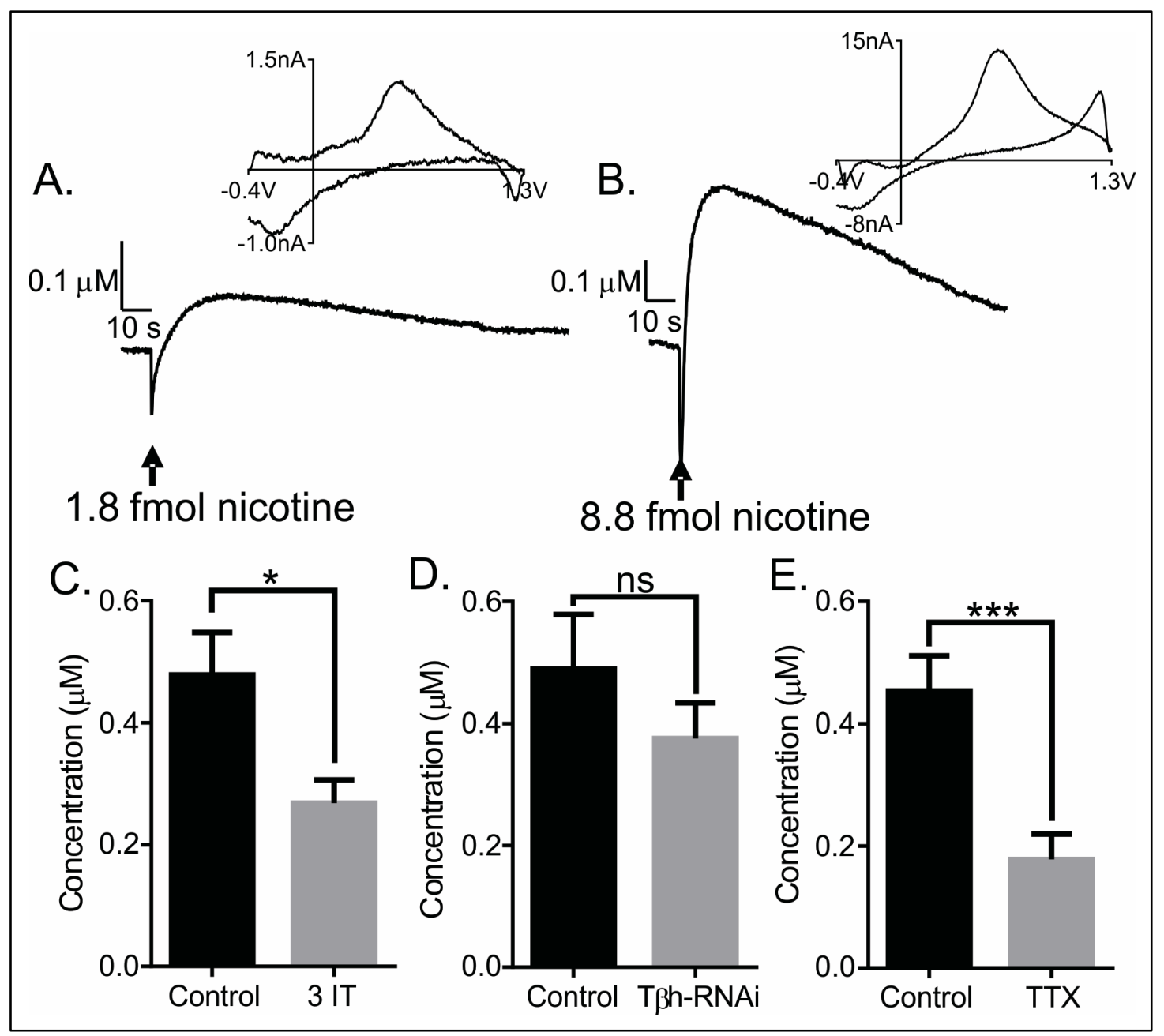

Figure 2.3. Nicotine stimulates dopamine release in the VNC, and the release is mediated by presynaptic nAChRs. (A-B) Current vs. time plot (bottom) and cyclic voltammogram (top) obtained upon nicotine stimulation in Drosophila larval VNC. (A) Example $1.8 \mathrm{fmol}$ stimulation. (B) Example 8.8 fmol stimulation. (C) In flies fed dopamine synthesis inhibitor, 3 iodotyrosine, the current response is significantly lower (unpaired $t$-test, $p=0.0345, n=5-6$ ), which confirms that the response is due to dopamine. (D) Nicotine-stimulated response is not significantly different than control in flies with a knockdown of octopamine synthesis (Tdc2-GAL4; UAS-RNAi ${ }^{T \beta H}$ ) (unpaired ttest, $p=0.3250, n=4$ ). (E) In the presence of sodium channel inhibitor, tetrodotoxin, the current response is significantly lower than control (paired $t$-test, $p=0.0007, n=7$ ), which shows that the release is mediated by presynaptic nAChRs.

Nicotine-stimulated release was measured repeatedly with 5 minute interstimulation times. The concentration of dopamine for each stimulation increases significantly and there is an effect of the amount of nicotine applied (Fig. 2.4A, 2-way ANOVA, significant effects of amount of nicotine and stimulation number, $p=0.0011$ for nicotine concentration, $p<0.0001$ for 
stimulation number, and no significant interaction, $p=0.97$ ). All of the higher dose stimulations elicited more dopamine than the lower dose stimulations (Fig. 2.4A, Sidak's post-test, $p<0.05$ for all). The increase in release is better visualized when the data is normalized to the first stimulation (Fig. 2.4B). With the normalized data, there are again main effects of nicotine amount and stimulation number on evoked dopamine release (2-way ANOVA, $p<0.0001$ for stimulation number and $p<0.01$ for nicotine dose, interaction $p<0.001$ ). Normalized responses with $1.8 \mathrm{fmol}$ stimulation are significantly larger than the $8.8 \mathrm{fmol}$ responses from $6^{\text {th }}$ to $9^{\text {th }}$ stimulations (Sidak's post-test, $\mathrm{p}<0.05$ ). Sensitivity of nAChRs increased to nicotine upon subsequent stimulations, which is more apparent with lower amount of nicotine. 


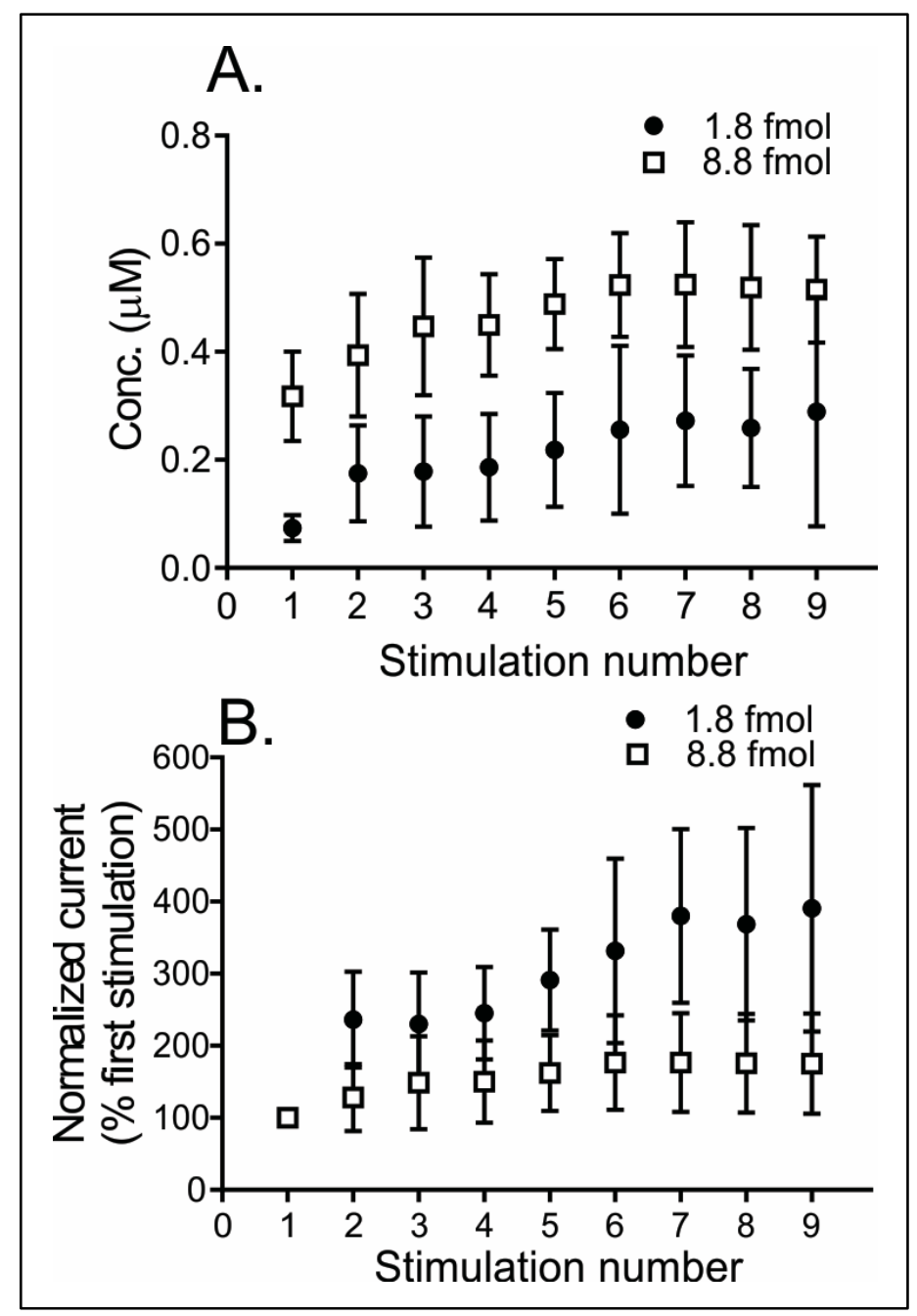

Figure 2.4. Repeated stimulations with nicotine. (A) The effect of repeated stimulations, 5 min apart, on the concentration of dopamine measured with $1.8 \mathrm{fmol}$ or $8.8 \mathrm{fmol}$ nicotine stimulation (2-way ANOVA, significant main effects of amount of nicotine and stimulation number, $p=0.0011$ for nicotine concentration, $p<0.0001$ for stimulation number, and no significant interaction, $p=0.97, n$ $=6$ ). $8.8 \mathrm{fmol}$ nicotine stimulation evokes more dopamine than $1.8 \mathrm{fmol}$ nicotine (Sidak's post-test, $p$ $<0.05$ for all). (B) Normalized current for repeated stimulations, which also shows main effects of nicotine amount and stimulation number on the response (2-way ANOVA, $p<0.0001$ for stimulation number and $p<0.01$ for nicotine dose, interaction $p<0.001$ ). There is a greater increase with 1.8 fmol than $8.8 \mathrm{fmol}$ nicotine (Sidak's post-test), which suggests that the increase in sensitivity to nicotine occurs faster and reaches maximal level faster with higher amounts of nicotine.

\subsubsection{Neonicotinoid-stimulated dopamine release in Drosophila melanogaster larval VNC}

Two different neonicotinoids, nitenpyram and imidacloprid, were tested to determine the extent to which they evoked dopamine release. A higher amount 
of nitenpyram ( $2.2 \mathrm{fmol})$ was used compared to imidacloprid ( $1.8 \mathrm{fmol})$ because imidacloprid is more potent in killing flies ${ }^{24}$ Stimulated dopamine release by both neonicotinoids $(0.7+/-0.1 \mu \mathrm{M}$ dopamine for nitenpyram and $0.32+/-0.04 \mu \mathrm{M}$ for imidacloprid) had similar characteristics to nicotine-stimulated release (Fig. 2.5A and C). The cyclic voltammogram profile of the release was indicative of dopamine, and in larvae fed with 3-iodotyrosine, dopamine release was significantly lower with stimulation by nitenpyram (unpaired t-test, $p=0.0284, \mathrm{n}=$ 5) or imidacloprid (unpaired t-test, $p=0.0036, \mathrm{n}=5$ ) (Fig. 2.5B and D). The neonicotinoid-evoked response was longer lasting $\left(t_{1 / 2}=86+/-7 \mathrm{~s}\right.$ for nitenpyram and $121+/-20 \mathrm{~s}$ for imidacloprid) than acetylcholine-stimulated response $\left(\mathrm{t}_{1 / 2}=\right.$ $19 \mathrm{~s}$, one-way ANOVA, $p<0.05, \mathrm{n}=7$ ). The $\mathrm{t}_{1 / 2}$ for imidacloprid-evoked response was also significantly higher than nicotine-stimulated response (oneway ANOVA, $p<0.05, \mathrm{n}=7$ ). 


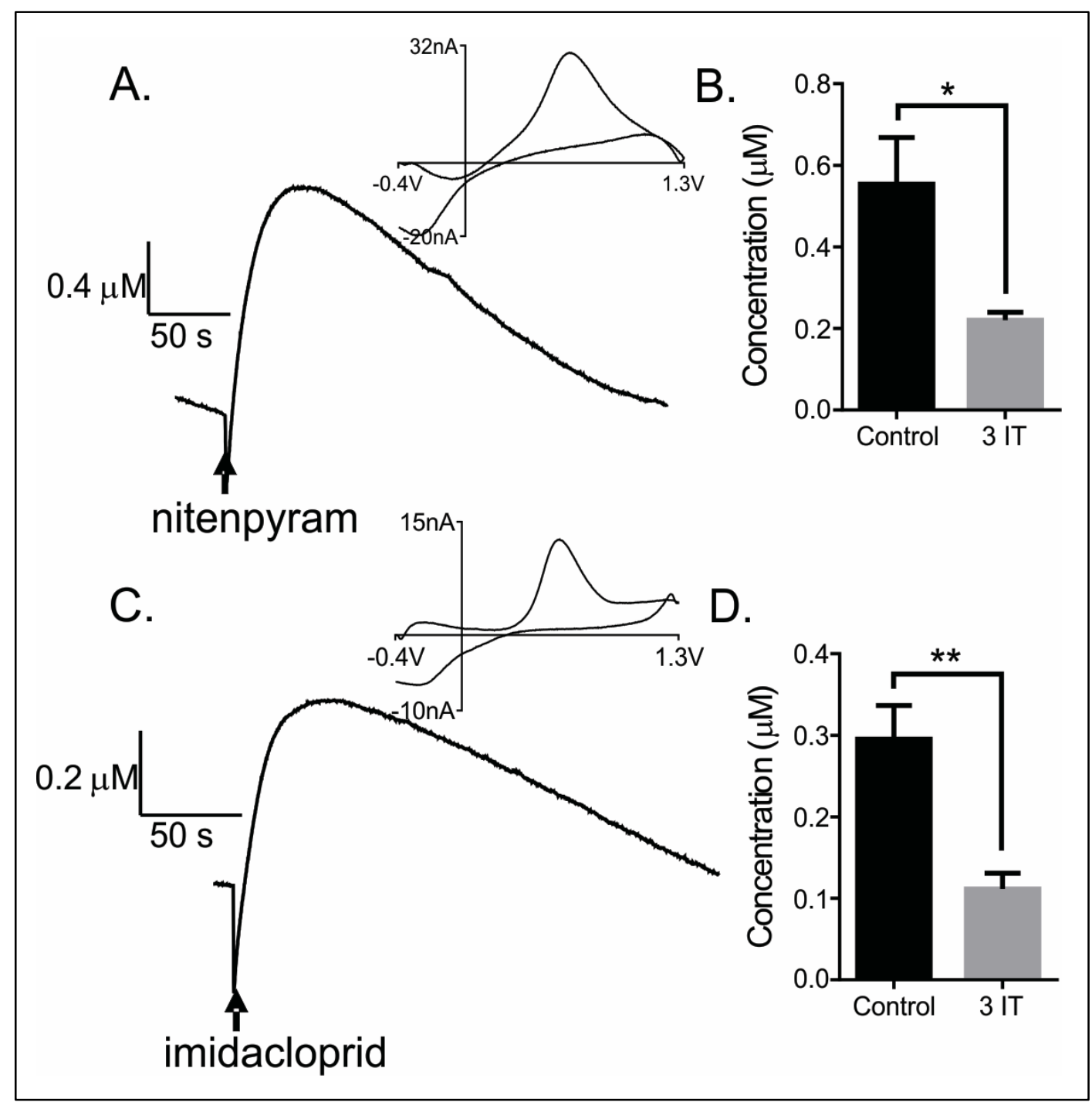

Figure 2.5. Neonicotinoids nitenpyram and imidacloprid stimulated release in Drosophila melanogaster ventral nerve cord (VNC). (A) $2.2 \mathrm{fmol}$ nitenpyram and (C) $1.8 \mathrm{fmol}$ imidacloprid evokes current response in the VNC, the cyclic voltammogram of which indicate dopamine. (B) Nitenpyram (unpaired t-test, $p=0.0284, n=5$ ) and (D) Imidacloprid-stimulated release is significantly lower in flies fed 3-iodotyrosine, confirming that the response is due to dopamine (unpaired t-test, $p=$ 0.0036, $n=5)$.

\subsubsection{Nicotine and neonicotinoid stimulated release in $a$ and $\beta$ nAChR subunit mutants}

Perry et. al have identified mutations in the a and $\beta$ subunits of Drosophila $\mathrm{nAChR}$ that confer reduced sensitivity to the neonicotinoids imidacloprid and nitenpyram. ${ }^{24}$ To test if nicotine-stimulated release is affected by the mutations, 
nicotine was puffed into the VNCs of Drosophila with a1 (EMS1) or $\beta 2$ (EMS2) subunit mutations (Fig. 2.6A). The release upon nicotine stimulation was not significantly different (one-way ANOVA, $p=0.7625, \mathrm{n}=4$ ), which suggests that effect of nicotine stimulation remains unchanged in these mutants.

To study the effect of neonicotinoid stimulations in the $\alpha 1$ (EMS1) and $\beta 2$ (EMS2) mutants, nitenpyram or imidacloprid were puffed into the VNCs. The dopamine release in the mutant VNCs was significantly lower than control for both nitenpyram (one-way ANOVA, $p=0.0010, n=4-6$ ) and imidacloprid stimulation (one-way ANOVA, $p<0.0001, \mathrm{n}=5$ ) (Fig. 2.6B and $\mathrm{C}$ ), suggesting that the neonicotinoid binding sites are affected in these mutants.

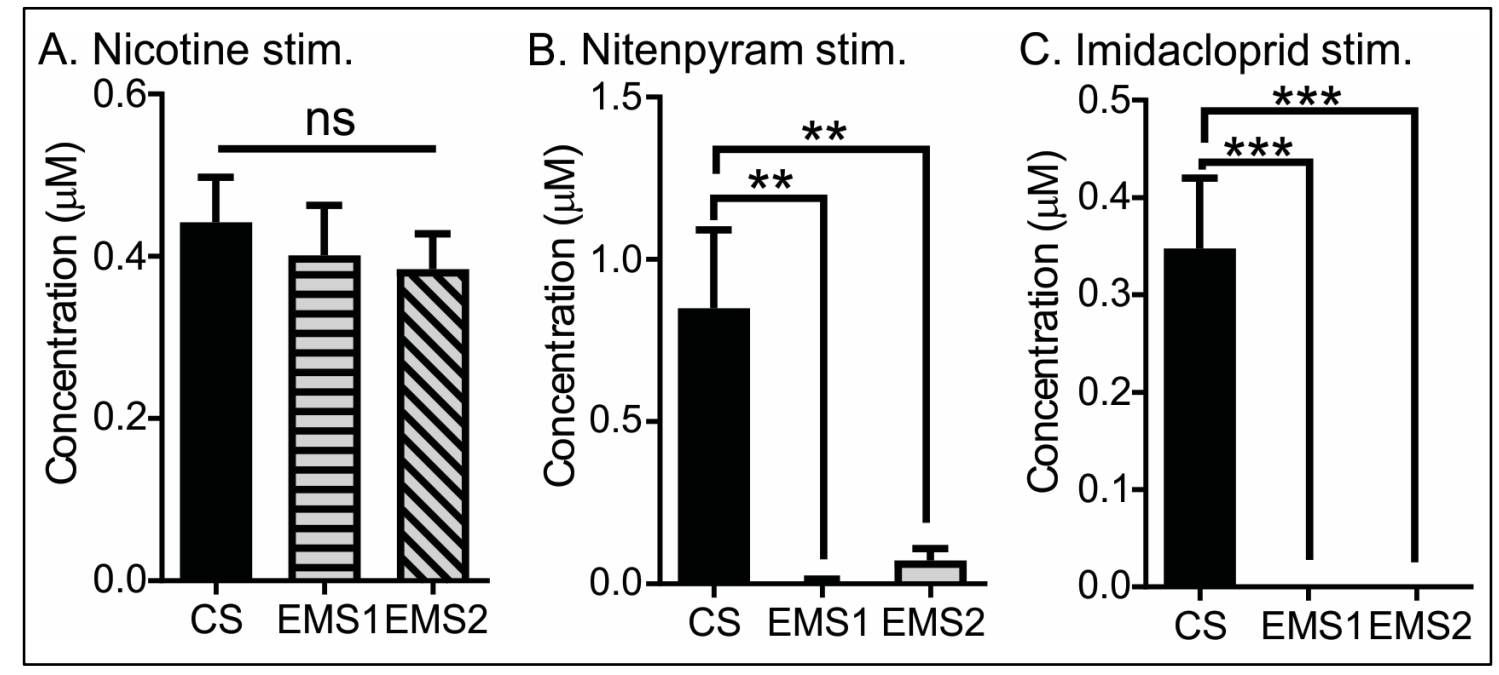

Figure 2.6. Effect of nAChR mutations on nicotine and neonicotinoid-stimulated response. (A) In flies with nAChR subunits a1 (EMS 1) or $\beta 2$ (EMS 2) mutations, nicotine stimulation evokes release that is not significantly different than in wild type Canton $S$ (CS) flies (one-way ANOVA, $p=0.7308, n$ = 4-7). (B) Nitenpyram (one-way ANOVA, $p=0.0010, n=4-6$ ) and (C) Imidacloprid-stimulated response is significantly lower in Drosophila with mutations in the nAChR subunits (one-way ANOVA, $p<0.0001, n=5$ ). 


\subsection{Discussion}

Dopamine regulates behavioral responses to nicotine in Drosophila, ${ }^{27}$ but neurochemical measurements of dopamine after nicotine stimulation had not been made. Here, we demonstrate for the first time that the stimulation of nAChRs causes dopamine release in Drosophila melanogaster larval VNC. These results are similar to findings in rodents that acetylcholine evokes dopamine. ${ }^{27,35}$ Acetylcholine, nicotine, and neonicotinoid insecticides evoked dopamine release in the Drosophila CNS and this response was mediated by nAChRs, and not mAchRs. All previous work in Drosophila larvae had used optogenetics or $\mathrm{P} 2 \mathrm{X}_{2}$ channels to stimulate dopamine release, which requires a channel to be expressed in specific neurons using genetics. ${ }^{28,29}$ Acetylcholine or nicotine stimulation evoke endogenous dopamine release, without needing to genetically modify the organism. Mutations in the subunits of the nAChRs affect neonicotinoid evoked dopamine release without changing the response to nicotine. Thus, dopamine measurements in Drosophila are an easy way to study the effects of nicotinic subunit mutations in causing excitatory responses.

\subsubsection{Acetylcholine-stimulated dopamine release}

Acetylcholine is the most abundant excitatory neurotransmitter in the CNS of insects ${ }^{1}$ and plays a role in learning and memory formation. ${ }^{3,36}$ Dopamine is another major neurotransmitter that mediates many functions in Drosophila, including learning and memory ${ }^{37}$ and modulates cholinergic transmission in Drosophila neuronal cultures. ${ }^{38}$ Here, we discovered that acetylcholine 
stimulation evokes a strong release of dopamine in the Drosophila larval VNC. The shape of the cyclic voltammogram and significantly lower response in Drosophila fed with 3-IT verify that the response is due to dopamine release. Because nAChR mediated octopamine release has been reported in adult fly brain preparations, ${ }^{39}$ we tested flies with reduced octopamine synthesis and found no change in the evoked dopamine. The electrode placement for measuring from dopamine neurons is towards the thoracic region, away from the abdominal ganglia where octopamine has been measured. ${ }^{40}$ Thus, this experiment was optimized for measuring acetylcholine receptor mediated dopamine release, although future studies could vary the electrode placement to target the abdominal segments of the neuropil to see if octopamine was released by acetylcholine.

Acetylcholine-stimulated dopamine release in flies is consistent with previous results in rodents, where it is well established that $n A C h R s$ are located presynaptically on dopamine neurons ${ }^{41}$ and modulate dopamine release. ${ }^{35}$ In rodents, mAchRs do not regulate dopamine release but nAChRs do. ${ }^{15}$ Similarly, in flies, acetylcholine-stimulated release was significantly lower in the presence of nAChR inhibitor a-BTX, but not different with the mAchR inhibitor atropine. Blocking nAChRs and decreasing release implies that the nAChRs are presynaptically located on dopamine terminals and control dopamine release. The similarities in release indicate a conserved mechanism of dopamine 
stimulation between flies and rodents, demonstrating that flies are a good model system to study nAChR mediated effects.

Stimulated dopamine release in Drosophila larval VNC has been previously studied with transgenic fruit flies that express either light or ATP sensitive ion channels in dopaminergic, serotoninergic, or octopaminergic cells. 29, 40, 42 Release using optogenetic stimulation was higher; a $7 \mathrm{~s}$ blue light stimulation evoked average of $810+/-60 \mathrm{nM}$ dopamine, compared to the $430+/-$ $40 \mathrm{nM}$ with acetylcholine. ${ }^{29} \mathrm{The}_{1 / 2}$ for blue light-stimulated release was $7 \mathrm{~s}$, which is shorter than $19 \mathrm{~s}$ observed for acetylcholine-stimulated release. The concentration of dopamine release and clearance time with acetylcholine were more similar to flies that were modified to express the $\mathrm{P} 2 \mathrm{X}_{2}$ channel, which was activated with exogenously applied ATP. ${ }^{43}$ Puffing on the stimulus likely leads to longer activation and longer signals. While optogenetic methods allow specific stimulation because the channel is expressed in only one cell type, the disadvantage is that the flies must be genetically altered to express the channel using the GAL4-UAS system. To test the effects of other genetic mutations on dopamine release, it can be difficult to also express the mutation and the optogenetic channel in the same line. Thus, using acetylcholine, a natural neurotransmitter, is advantageous because dopamine release can be stimulated in any fly, including mutant flies, without needing genetic alterations to express an exogenous ion channel. 


\subsubsection{Nicotine-stimulated dopamine release}

Nicotine is a natural insecticide that acts on nAChRs, overstimulating the nervous system of insects. The dopamine system is important for mediating the effects of nicotine in Drosophila. ${ }^{27}$ For example, Drosophila exposed to volatilized nicotine show hyperactivity, spasmodic movements, and impaired ability to negatively geotax, and these behaviors are reduced by about 35\% in flies fed with $3-I T .{ }^{27}$ We hypothesized that nicotine would elicit dopamine release through nAChRs and indeed, nicotine evoked dopamine release in a similar manner to acetylcholine. The sodium channel blocker, TTX, lowered nicotine- stimulated release significantly, demonstrating that release is exocytotic and suggesting that the nAChRs are presynaptic. One recent study found that simultaneous stimulation of two pathways: an acetylcholine pathway, mediated by nAChRs and a glutamatergic pathway, mediated by NMDA receptors, causes dopamine release in the mushroom bodies of adult Drosophila. ${ }^{44}$ In that study, the dopamine neuron was postsynaptic to the two inputs, but here only acetylcholine is needed and there is no evidence for any activation other than simple presynaptic nAChRs.

There are some differences between acetylcholine and nicotine mediated dopamine release. The $t_{1 / 2}$ for acetylcholine mediated release is much smaller than that for nicotine mediated release. Acetylcholine, unlike nicotine, is a natural neurotransmitter that can be metabolized by acetylcholinesterase in the

CNS of insects; hence, it would be rapidly cleared from the extracellular space. ${ }^{45}$ Nicotine, on the other hand, is expected to be metabolized slower and mostly 
cleared by diffusion, and give a longer lasting response. ${ }^{46}$ The result agrees with previous observations of nicotine-stimulated release lasting much longer than acetylcholine-stimulated release in the rat substantia nigra and ventral tegmental area. $^{47}$ Similarly, the amount of acetylcholine (1 $\mathrm{mM}$ in pipette, 1.8 pmol injected) used for stimulations needs to be much higher than for nicotine (1-5 $\mu \mathrm{M}$ in pipette, $1.8-8.8 \mathrm{fmol}$ injected), likely because acetylcholine is rapidly metabolized and has a lower binding affinity to nAChRs than nicotine. ${ }^{33}$

There are also differences in the stability of release with repeated stimulations for nicotine and acetylcholine. Acetylcholine-stimulated dopamine release was stable when repeated every 5 minutes, while nicotine-stimulated dopamine release increased with more stimulations. The percentage increase was largest for low amounts of nicotine applied, where the release never plateaued even when 9 stimulations were performed. With higher amounts of nicotine applied, the concentration of dopamine evoked was higher, but the percentage increase during subsequent stimulations was not as large and release plateaus after a few stimulations. Possible mechanisms for the increase in dopamine release include desensitization of nAChRs on GABA (i.e inhibitory) neurons ${ }^{48}$ or upregulation of nAChRs. ${ }^{49}$ Both of these mechanisms have been demonstrated in rats, but nicotine exposure to upregulate nAChRs was on the time course of 8-24 hours, not 1 hour, the time course of this experiment. ${ }^{49}$ With $8.8 \mathrm{fmol}$ nicotine applied, the desensitization and upregulation is expected to occur faster than with $1.8 \mathrm{fmol}$, where it occurs more gradually, so the increase 
continues for more stimulations. Studies of behavioral sensitization to nicotine in rats have found that repeated doses are needed to induce sensitization with nicotine, and these doses should be delivered quickly (i.e. within seconds) and not once a day. ${ }^{50}$ Indeed, with mammalian smoking addictions, inhalation is repeated multiple times ${ }^{51}$ and insects may eat a plant multiple times, which would lead to sensitization compared to one single exposure. There are Drosophila models with increased nicotine sensitivity, and future studies can investigate if these flies are already sensitized and if they have a difference in release patterns with repeated stimulations. ${ }^{52}$

\subsubsection{Neonicotinoid-stimulated release}

Neonicotinoids are synthetic insecticides analogous in function to nicotine. They overstimulate and desensitize nAChRs, causing paralysis and death of insects. ${ }^{7}$ Similar to nicotine, stimulations with neonicotinoids imidacloprid or nitenpyram caused a long lasting dopamine release compared to acetylcholinestimulated response, and evoked dopamine was lower in larvae fed with 3-IT. However, the response for imidacloprid stimulation was even longer than the nicotine evoked response. A previous report found that neuronal firing evoked by imidacloprid fell more gradually than that evoked by nicotine. ${ }^{53}$ Thus, imidacloprid may activate nAChRs longer than nicotine, which could be a factor for its effectiveness as an insecticide.

In mutant Drosophila lines that have increased resistance to nitenpyram and imidacloprid, neonicotinoid stimulation caused almost no dopamine release. 
The resistant lines have a significantly lower mortality rate compared to wild type in the presence of the neonicotinoids in the growth medium. ${ }^{24}$ These lines have mutations in the nAChR $\alpha 1$ (EMS 1) or $\beta 2$ (EMS 2) subunits and both mutations were quite effective, as very little dopamine release was stimulated by either neonicotinoid. ${ }^{24}$ While the EMS lines had reduced sensitivity to neonicotinoids, they maintained their sensitivity to nicotine, showing that the neonicotinoids likely have different binding sites at nAChRs. Indeed, previous studies demonstrated that nicotine and neonicotinoids have different interactions with acetylcholine binding proteins, ${ }^{54}$ and different effects in resistant insects. ${ }^{55}$ Neurochemical measurements in Drosophila are useful to study the downstream effects of $\mathrm{nAChR}$ subunit mutations on neurotransmitter release. Mutations in $\mathrm{nAChR}$ subunits confer resistance to insecticides, which is an important concern in agriculture. Our results demonstrate that dopamine stimulation is dramatically reduced in neonicotinoid resistant flies and other mutations could be studied to determine if they result in neonicotinoid resistance. Thus, dopamine measurements in Drosophila are useful for understanding the target specificity of neonicotinoid insecticides to different nAChRs. ${ }^{55,56}$

\subsection{Conclusions}

We demonstrated for the first time that the nAChR agonists acetylcholine, nicotine, and neonicotinoids stimulate dopamine release in the Drosophila larval VNC. The release is mediated by presynaptic nAChRs, and not mAchRs, and is sensitive to tetrodotoxin, indicating release is exocytotic. Nicotine and 
neonicotinoids stimulate dopamine release that lasts longer than acetylcholinestimulated release, likely due to higher affinities and fewer mechanisms for their metabolism and clearance. Neonicotinoid-stimulated response is significantly lower in Drosophila strains that are resistant to the neonicotinoids and have mutations in a1 or $\beta 2 \mathrm{nAChR}$ subunits. Nicotine-stimulated response, however, is not significantly different than controls in the mutant strains. Thus, Drosophila is an important model organism to study the effects of nAChR mutations or agonists on dopamine release and may yield important information about the pathways of acetylcholine regulation of dopamine release.

\subsection{Experimental Section}

\subsubsection{Chemicals}

All chemicals were purchased from Sigma-Aldrich (St. Louis, MO), and solutions were prepared in Milli Q water (Millipore, Billerica, MA) unless noted otherwise. Electrode calibrations were performed in phosphate buffer solution (PBS; $131.25 \mathrm{mM} \mathrm{NaCl}, 3.0 \mathrm{mM} \mathrm{KCl}, 10.0 \mathrm{mM} \mathrm{NaH}_{2} \mathrm{PO}_{4}, 1.2 \mathrm{mM} \mathrm{MgCl}_{2}, 2.0 \mathrm{mM}$ $\mathrm{Na}_{2} \mathrm{SO}_{4}$, and $1.2 \mathrm{mM} \mathrm{CaCl}_{2}$ ) with $\mathrm{pH}$ adjusted to 7.4 , which was made once a month and stored at $4{ }^{\circ} \mathrm{C}$. To make the larval dissection buffer, $11.1 \mathrm{mM}$ glucose and $5.3 \mathrm{mM}$ trehalose were added to the PBS buffer on the day of the experiment. A $10 \mathrm{mM}$ stock solution of dopamine was prepared in $0.1 \mathrm{M} \mathrm{HClO}_{4}$ once a month and stored at $4{ }^{\circ} \mathrm{C}$. Diluted dopamine solution for electrode calibration was prepared in PBS buffer the day of the experiment. 
Acetylcholine chloride (1 mM) solution was prepared daily in PBS. 3iodotyrosine was mixed with water and standard cornmeal food to make total concentration of $10 \mathrm{mg} / \mathrm{mL}$. Larvae were fed with $3 \mathrm{IT}$ food for 2 days prior to experimentation. (-)-Nicotine ditartrate (Tocris Bioscience, Bristol, UK) was prepared in $1 \mu \mathrm{M}$ or $5 \mu \mathrm{M}$ solution in PBS on the day of experiment. Imidacloprid and nitenpyram were purchased from ChemService (West Chester, PA). Stocks of imidacloprid (1 $\mathrm{mM}$ in DMSO) and nitenpyram (1 $\mathrm{mM}$ in water) were diluted (to $1 \mu \mathrm{M}$ for imidacloprid, $1.25 \mu \mathrm{M}$ for nitenpyram) in PBS buffer.

Atropine $(4 \mu \mathrm{M})$, alpha-bungarotoxin $(8 \mu \mathrm{M})$ and tetrodotoxin $(2 \mu \mathrm{M})$ were prepared in PBS buffer. To add drug to the VNC, $1 \mathrm{~mL}$ of the respective solution was added to the Petri dish that contained $3 \mathrm{~mL}$ of dissection buffer.

\subsubsection{Drosophila and VNC Preparation}

Drosophila melanogaster strains were obtained from Bloomington Stock Center: Canton S (stock \#64349); GAL4 driver on octopaminergic/tyraminergernic neurons (Tdc2-GAL4, \#9313); and UAS-RNAi on octopaminergic neurons, (UAS-RNAi ${ }^{\mathrm{T} H \mathrm{H}}$ \#27667). Strains resistant to neonicotinoids (EMS1 and EMS2) were obtained from Dr. Trent Perry at the University of Melbourne, Australia. Drosophila melanogaster stocks were maintained and crossed as described before. ${ }^{28}$

Larvae were dissected in modified PBS as previously described. ${ }^{28}$ Briefly, the central nervous system was dissected out from a third instar larva using fine tweezers, the optic lobes were cut off, and the VNC was transferred to the lid of a 
Petri dish with $3 \mathrm{~mL}$ of the dissection buffer in it. The opposite end of the VNC was cut with fine scissors to facilitate micropipette insertion. ${ }^{57}$

\subsubsection{Electrochemical Setup and Data Analysis}

CFMEs were fabricated with $7 \mu \mathrm{m}$ diameter T-650 carbon fibers (Cytec Engineering Materials, West Patterson, $\mathrm{NJ}$ ) in a $1.2 \mathrm{~mm}$ o.d. glass capillary (A-M systems, Carlsburg, WA) pulled to a tip. CFMEs were cut to $50-75 \mu \mathrm{m}$ to form cylindrical electrodes. Data were collected with Dagan Chem-Clamp potentiostat (Dagan, Minneapolis, MN, $n=0.01$ headstage), $\mathrm{PCl} 6711$ and 6052 computer interface cards (National Instruments, Austin, TX), and a home-built breakout box. Tar Heel CV software (gift of Mark Wightman, University of North Carolina) was used for data collection and analysis. Electrodes were precalibrated with 1 $\mu \mathrm{M}$ dopamine in a flow cell.

The VNC and electrode were viewed under a 40X water immersion lens (Carl Zeiss Microscopy, LLC, Thornwood, NY, USA) and the electrode was inserted in the neuropil 4-5 segments from the cut end using a micromanipulator (Narshige N-MMN-1 and N-MMO-202ND). The potential was applied between the $\mathrm{CFME}$ and a $\mathrm{Ag} / \mathrm{AgCl}$ reference electrode in the bath. A picospritzing pipet was made with the same glass capillary and vertical puller used to fabricate CFMEs. After being pulled, the pipet tips were trimmed. The pipet was filled with acetylcholine, nicotine or neonicotinoid and inserted into the VNC to pressure eject the agonists using Picospritzer III instrument (Parker Hannifin, Fairfield, NJ). The pipet was calibrated by measuring the diameter of liquid ejected in oil at a 
set pressure and ejection time.

All statistics were performed with GraphPad Prism 6 (GraphPad Software, Inc., La Jolla, CA). Significance was measured with a 95\% confidence interval. Graphs are shown as mean \pm standard error of the mean. For power analysis, mean acetylcholine-stimulated dopamine release of $0.45 \mu \mathrm{M}$, a $35 \%$ lower null hypothesis mean $(0.29 \mu \mathrm{M})$ and standard deviation of 0.12 was used, which meant an $n$ value of 5 was needed. Power analysis for nicotine-stimulated dopamine release used true mean of $0.50 \mu \mathrm{M}$, a $35 \%$ lower null hypothesis mean $(0.33 \mu \mathrm{M})$ and standard deviation of 0.13 , which also predicted a $n=5$ was needed. 


\subsection{References}

[1] Hoy, R. R. (1988) Arthropod Brain - Its Evolution, Development, Structure, and Functions - Gupta,Ap, Science 240, 668-668.

[2] Gauglitz, S., and Pfluger, H. J. (2001) Cholinergic transmission via central synapses in the locust nervous system, J Comp Physiol A 187, 825-836.

[3] El Hassani, A. K., Dacher, M., Gary, V., Lambin, M., Gauthier, M., and Armengaud, C. (2008) Effects of sublethal doses of acetamiprid and thiamethoxam on the behavior of the honeybee (Apis mellifera), Arch Environ Con Tox 54, 653-661.

[4] Fukuto, T. R. (1990) Mechanism of Action of Organophosphorus and Carbamate Insecticides, Environ Health Persp 87, 245-254.

[5] Matsuda, K., Buckingham, S. D., Kleier, D., Rauh, J. J., Grauso, M., and Sattelle, D. B. (2001) Neonicotinoids: insecticides acting on insect nicotinic acetylcholine receptors, Trends Pharmacol Sci 22, 573-580.

[6] Brown, L. A., Ihara, M., Buckingham, S. D., Matsuda, K., and Sattelle, D. B. (2006) Neonicotinoid insecticides display partial and super agonist actions on native insect nicotinic acetylcholine receptors, J Neurochem 99, 608615.

[7] Plumlee, K. H. e. (2004) Clinical Veterinary Toxicology, Mosby, St. Louis, Mo.

[8] Breer, H., and Sattelle, D. B. (1987) Molecular-Properties and Functions of Insect Acetylcholine-Receptors, J Insect Physiol 33, 771-790.

[9] Salvaterra, P. M., and Foders, R. M. (1979) [2-Alpha-Bungarotoxin-I-125 and [Quinuclidinylbenzilate-H-3 Binding in Central Nervous Systems of Different Species, J Neurochem 32, 1509-1517.

[10] Breer, H. (1981) Properties of putative nicotinic and muscarinic cholinergic receptors in the central nervous system of Locusta migratoria, Neurochem Int 3, 43-52.

[11] Lummis, S. C., and Sattelle, D. B. (1985) Binding of N-[propionyl$3 \mathrm{H}$ ]propionylated alpha-bungarotoxin and L-[benzilic-4,4'-3H] quinuclidinyl benzilate to CNS extracts of the cockroach Periplaneta americana, Comp Biochem Physiol C 80, 75-83. 
[12] Harrow, I. D., and Sattelle, D. B. (1983) Acetylcholine-Receptors on the Cell Body Membrane of Giant Interneuron-2 in the Cockroach, PeriplanetaAmericana, J Exp Biol 105, 339-350.

[13] Rohrbough, J., and Broadie, K. (2002) Electrophysiological analysis of synaptic transmission in central neurons of Drosophila larvae, $J$ Neurophysiol 88, 847-860.

[14] Rice, M. E., and Cragg, S. J. (2004) Nicotine amplifies reward-related dopamine signals in striatum, Nat Neurosci 7, 583-584.

[15] Zhou, F. M., Liang, Y., and Dani, J. A. (2001) Endogenous nicotinic cholinergic activity regulates dopamine release in the striatum, Nat Neurosci 4, 1224-1229.

[16] Corringer, P. J., Le Novere, N., and Changeux, J. P. (2000) Nicotinic receptors at the amino acid level, Annu Rev Pharmacol 40, 431-458.

[17] Gill, J. K., Savolainen, M., Young, G. T., Zwart, R., Sher, E., and Millar, N. S. (2011) Agonist activation of alpha 7 nicotinic acetylcholine receptors via an allosteric transmembrane site, P Natl Acad Sci USA 108, 5867-5872.

[18] Benke, D., and Breer, H. (1989) Comparison of Acetylcholine and AlphaBungarotoxin Binding-Sites in Insects and Vertebrates, Comp Biochem Phys C 94, 71-80.

[19] Exley, R., and Cragg, S. J. (2008) Presynaptic nicotinic receptors: a dynamic and diverse cholinergic filter of striatal dopamine neurotransmission, Brit $J$ Pharmacol 153, S283-S297.

[20] Lansdell, S. J., Collins, T., Goodchild, J., and Millar, N. S. (2012) The Drosophila nicotinic acetylcholine receptor subunits Dalpha5 and Dalpha7 form functional homomeric and heteromeric ion channels, Bmc Neurosci $13,73$.

[21] Kao, P. N., and Karlin, A. (1986) Acetylcholine-Receptor Binding-Site Contains a Disulfide Cross-Link between Adjacent Half-Cystinyl Residues, J Biol Chem 261, 8085-8088.

[22] Koo, H. N., An, J. J., Park, S. E., Kim, J. I., and Kim, G. H. (2014) Regional susceptibilities to 12 insecticides of melon and cotton aphid, Aphis 
gossypii (Hemiptera: Aphididae) and a point mutation associated with imidacloprid resistance, Crop Prot 55, 91-97.

[23] Thany, S. H., Lenaers, G., Raymond-Delpech, V., Sattelle, D. B., and Lapied, B. (2007) Exploring the pharmacological properties of insect nicotinic acetylcholine receptors, Trends Pharmacol Sci 28, 14-22.

[24] Perry, T., Heckel, D. G., McKenzie, J. A., and Batterham, P. (2008) Mutations in $\mathrm{D}$ alpha 1 or $\mathrm{D}$ beta 2 nicotinic acetylcholine receptor subunits can confer resistance to neonicotinoids in Drosophila melanogaster, Insect Biochem Molec 38, 520-528.

[25] Wu, P. P., Ma, D. D., Pierzchala, M., Wu, J., Yang, L. C., Mai, X. P., Chang, X. Y., and Schmidt-Glenewinkel, T. S. (2005) The Drosophila acetylcholine receptor subunit $D$ alpha 5 is part of an alpha-bungarotoxin binding acetylcholine receptor, J Biol Chem 280, 20987-20994.

[26] Perry, T., McKenzie, J. A., and Batterham, P. (2007) A Dalpha6 knockout strain of Drosophila melanogaster confers a high level of resistance to spinosad, Insect Biochem Mol Biol 37, 184-188.

[27] Bainton, R. J., Tsai, L. T. Y., Singh, C. M., Moore, M. S., Neckameyer, W. S., and Heberlein, U. (2000) Dopamine modulates acute responses to cocaine, nicotine and ethanol in Drosophila, Curr Biol 10, 187-194.

[28] Privman, E., and Venton, B. J. (2015) Comparison of dopamine kinetics in the larval Drosophila ventral nerve cord and protocerebrum with improved optogenetic stimulation, $J$ Neurochem 135, 695-704.

[29] Vickrey, T. L., Condron, B., and Venton, B. J. (2009) Detection of endogenous dopamine changes in Drosophila melanogaster using fastscan cyclic voltammetry, Analytical chemistry 81, 9306-9313.

[30] Couton, L., Mauss, A. S., Yunusov, T., Diegelmann, S., Evers, J. F., and Landgraf, M. (2015) Development of Connectivity in a Motoneuronal Network in Drosophila Larvae, Curr Biol 25, 568-576.

[31] Taillebois, E., Beloula, A., Quinchard, S., Jaubert-Possamai, S., Daguin, A., Servent, D., Tagu, D., Thany, S. H., and Tricoire-Leignel, H. (2014) Neonicotinoid Binding, Toxicity and Expression of Nicotinic Acetylcholine Receptor Subunits in the Aphid Acyrthosiphon pisum, Plos One 9. 
[32] Zheng, J. Q., Felder, M., Connor, J. A., and Poo, M. M. (1994) Turning of Nerve Growth Cones Induced by Neurotransmitters, Nature 368, 140-144.

[33] Jensen, A. A., Mikkelsen, I., Frolund, B., Brauner-Osborne, H., Falch, E., and Krogsgaard-Larsen, P. (2003) Carbamoylcholine homologs: Novel and potent agonists at neuronal nicotinic acetylcholine receptors, $\mathrm{Mol}$ Pharmacol 64, 865-875.

[34] Klink, R., d'Exaerde, A. D., Zoli, M., and Changeux, J. P. (2001) Molecular and physiological diversity of nicotinic acetylcholine receptors in the midbrain dopaminergic nuclei, J Neurosci 21, 1452-1463.

[35] Ding, J. B., Guzman, J. N., Peterson, J. D., Goldberg, J. A., and Surmeier, D. J. (2010) Thalamic Gating of Corticostriatal Signaling by Cholinergic Interneurons, Neuron 67, 294-307.

[36] Hasselmo, M. E. (2006) The role of acetylcholine in learning and memory, Curr Opin Neurobiol 16, 710-715.

[37] Scholz-Kornehl, S., and Schwarzel, M. (2016) Circuit Analysis of a Drosophila Dopamine Type 2 Receptor That Supports AnesthesiaResistant Memory, J Neurosci 36, 7936-7945.

[38] Yuan, N., and Lee, D. W. (2007) Suppression of excitatory cholinergic synaptic transmission by Drosophila dopamine D1-like receptors, Eur $J$ Neurosci 26, 2417-2427.

[39] Fuenzalida-Uribe, N., Meza, R. C., Hoffmann, H. A., Varas, R., and Campusano, J. M. (2013) nAChR-induced octopamine release mediates the effect of nicotine on a startle response in Drosophila melanogaster, $J$ Neurochem 125, 281-290.

[40] Pyakurel, P., Champaloux, E. P., and Venton, B. J. (2016) Fast-Scan Cyclic Voltammetry (FSCV) Detection of Endogenous Octopamine in Drosophila melanogaster Ventral Nerve Cord, Acs Chemical Neuroscience 7, 11121119.

[41] Azam, L., Winzer-Serhan, U. H., Chen, Y. L., and Leslie, F. M. (2002) Expression of neuronal nicotinic acetylcholine receptor subunit mRNAs within midbrain dopamine neurons, J Comp Neurol 444, 260-274. 
[42] Xiao, N., Privman, E., and Venton, B. J. (2014) Optogenetic Control of Serotonin and Dopamine Release in Drosophila Larvae, Acs Chemical Neuroscience 5, 666-673.

[43] Xiao, N., and Venton, B. J. (2015) Characterization of dopamine releasable and reserve pools in Drosophila larvae using ATP/P2X(2)-mediated stimulation, J Neurochem 134, 445-454.

[44] Ueno, K., Suzuki, E., Naganos, S., Ofusa, K., Horiuchi, J., and Saitoe, M. (2017) Coincident postsynaptic activity gates presynaptic dopamine release to induce plasticity in Drosophila mushroom bodies, Elife 6.

[45] Quinn, D. M. (1987) Acetylcholinesterase - Enzyme Structure, Reaction Dynamics, and Virtual Transition-States, Chem Rev 87, 955-979.

[46] Self, L. S., Guthrie, F. E., and Hodgson, E. (1964) Metabolism of Nicotine by Tobacco-Feeding Insects, Nature 204, 300-301.

[47] Klink, R., de Kerchove d'Exaerde, A., Zoli, M., and Changeux, J. P. (2001) Molecular and physiological diversity of nicotinic acetylcholine receptors in the midbrain dopaminergic nuclei, $J$ Neurosci 21, 1452-1463.

[48] Mansvelder, H. D., Keath, J. R., and McGehee, D. S. (2002) Synaptic mechanisms underlie nicotine-induced excitability of brain reward areas, Neuron 33, 905-919.

[49] Govind, A. P., Vezina, P., and Green, W. N. (2009) Nicotine-induced upregulation of nicotinic receptors: underlying mechanisms and relevance to nicotine addiction, Biochem Pharmacol 78, 756-765.

[50] Samaha, A. N., Yau, W. Y. W., Yang, P. W., and Robinson, T. E. (2005) Rapid delivery of nicotine promotes behavioral sensitization and alters its neurobiological impact, Biol Psychiat 57, 351-360.

[51] Hilario, M. R., Turner, J. R., and Blendy, J. A. (2012) Reward sensitization: effects of repeated nicotine exposure and withdrawal in mice, Neuropsychopharmacology 37, 2661-2670.

[52] Sanchez-Diaz, I., Rosales-Bravo, F., Reyes-Taboada, J. L., Covarrubias, A. A., Narvaez-Padilla, V., and Reynaud, E. (2015) The Esg Gene Is Involved in Nicotine Sensitivity in Drosophila melanogaster, Plos One 10. 
[53] Kimura-Kuroda, J., Komuta, Y., Kuroda, Y., Hayashi, M., and Kawano, H. (2012) Nicotine-Like Effects of the Neonicotinoid Insecticides Acetamiprid and Imidacloprid on Cerebellar Neurons from Neonatal Rats, Plos One 7.

[54] Tomizawa, M., Talley, T. T., Maltby, D., Durkin, K. A., Medzihradszky, K. F., Burlingame, A. L., Taylor, P., and Casida, J. E. (2007) Mapping the elusive neonicotinoid binding site, Proc Natl Acad Sci U S A 104, 9075-9080.

[55] Mota-Sanchez, D., Hollingworth, R. M., Grafius, E. J., and Moyer, D. D. (2006) Resistance and cross-resistance to neonicotinoid insecticides and spinosad in the Colorado potato beetle, Leptinotarsa decemlineata (Say) (Coleoptera : Chrysomelidae), Pest Manag Sci 62, 30-37.

[56] Georghiou, G. P. (1994) Principles of Insecticide Resistance Management, Phytoprotection 75, 51-59.

[57] Vickrey, T. L., Xiao, N., and Venton, B. J. (2013) Kinetics of the Dopamine Transporter in Drosophila Larva, Acs Chemical Neuroscience 4, 832-837. 
Chapter 3: Age-related changes in Drosophila melanogaster controls and Parkin or RNF-11 mutants 


\section{Chapter 3: Age-related changes in Drosophila melanogaster controls and Parkin or RNF-11 mutants}

\subsection{Abstract}

Parkinson's disease is a neurodegenerative disorder characterized by loss of dopaminergic neurons in the substantia nigra region of the brain, most commonly in the elderly population. When the disease is diagnosed an approximately $80 \%$ of dopaminergic cells have already died. It is therefore important to understand early changes in neurochemistry so the disease can be identified earlier. Drosophila melanogaster Parkinson disease (PD) models have been developed to study molecular mechanisms of the disease. Using fast scan cyclic voltammetry (FSCV) we studied age-related changes in acetylcholinestimulated dopamine release and dopamine tissue content in the CNS for control flies and a fly model Parkinson disease - Parkin-RNAi. We also tested a potential genetic target for PD treatment, RNF11-RNAi. We found a significant effect of age on evoked dopamine current with repeated short interval stimulations, as old flies had significantly less decrease of dopamine than mid-age flies. This effect of age was not seen in the Parkinson's disease model flies suggesting that these flies lose the ability to maintain dopamine signaling. There was a significant effect of age on the tissue content of RNF11-RNAi flies where old flies had a significantly higher dopamine tissue content than younger flies. There was also an effect of genotype when repeated stimulations were compared in old flies of the different genotype. This work shows that the Parkinson disease mutation 
does not have a dramatic effect on dopamine levels but that there are subtle changes in the ability to maintain release for repeated stimulations.

\subsection{Introduction}

Parkinson's disease is a neurodegenerative disorder that affects over 10 million people worldwide. ${ }^{1}$ It is a debilitating disease that affects mood, memory, sleep and motor functions. The disease is most common in people over the age of 50 , and the incidence of the disease increases with age. ${ }^{2}$ Most cases of Parkinson's disease are sporadic and causes of the disease are not well known although genetic, ${ }^{3}$ environmental, ${ }^{4}$ and interaction of environmental-genetic factors $^{5,6}$ increases the likelihood of developing the disease. Mutations in several genes such as a-synuclein ${ }^{7}, \operatorname{Parkin}^{8}, P I N K 1^{6}$ and $L R R K 2^{9}$ are linked to Parkinson's disease. Although the mutations are in different genes, the cellular effects of the disease are the same - loss of dopaminergic cells in the substantia nigra pars compacta in the midbrain. ${ }^{10}$ The symptoms of the disease develop when up to $80 \%$ of dopamine-producing cells have already died. ${ }^{11}$ The disease is diagnosed based on symptoms and responsiveness to Parkinson's drug treatment and there is no good method for early disease diagnosis. ${ }^{12}$ The treatment options available are only symptomatic, and there is no cure or disease modifying treatment available. Development of neuroprotective treatments has been slow mainly due to the limited knowledge of the molecular mechanism of the disease. Better understanding of the mechanism of dopaminergic cell loss 
and disease biomarkers is needed for better drug development and early diagnosis of the disease. Model organisms like Caenorhabditis elegans and Drosophila melanogaster have been used for high throughput genetic screening for better understanding of the molecular mechanisms of neurodegenerative diseases. ${ }^{13,14}$ However, the changes in neurochemistry caused by these genetic mutations in Parkinson disease are largely unknown.

Drosophila melanogaster has been extensively studied as model organism for Parkinson's disease. ${ }^{15-18}$ Fundamental aspects of dopamine synthesis, release, and signaling processes are conserved between humans and Drosophila. ${ }^{19}$ This makes it an excellent model organism to study the cellular, molecular, and neurochemical changes that accompany dopaminergic cell loss in Parkinson's disease. There are several genetic manipulation tools available for use in Drosophila that have been used to create mutations in Drosophila homologs for Parkin, PINK1, LRR2 and other Parkinson's disease genes. Parkinson's disease model Drosophila develop phenotype such as loss of mitochondrial integrity, muscle loss, ${ }^{20}$ locomotor defects, abnormal wing posture and defects in climbing. ${ }^{18}$ Similar symptoms such as mitochondria dysfunction, ${ }^{21}$ muscle weakness, ${ }^{22}$ and gait and postural problems ${ }^{23}$ occurs in patients with Parkinson's disease. In Drosophila, however, there is conflicting evidence on how much the different Parkinson's disease mutations affect the dopaminergic cells, as some Parkin mutants do not show a loss of dopaminergic cells bodies. ${ }^{24,25}$ Some Parkin mutants do have a degeneration of a subset of dopaminergic 
neurons such as the PPL1 or PPL2ab region and a decrease in dopamine synthesis enzyme tyrosine hydroxylase (TH). ${ }^{14,16,26}$ Parkinson's disease patients also have similar cellular effects. ${ }^{27}$ Drosophila can, therefore, be used to understand the neurochemical mechanism of Parkinson's disease.

RNF11 is expressed in neurons and is another protein linked to Parkinson's disease. ${ }^{28}$ There is a decreased expression of RNF11 in dopaminergic cells of Parkinson's disease patients, and RNF11 knockdown increases protection of dopaminergic cells in 6-OHDA Parkinson's disease models. ${ }^{29,}{ }^{30}$ We have shown that a knockdown of RNF11 gene using RNAi causes an increase in stimulated dopamine release and clearance in Drosophila larvae. ${ }^{31}$ However, the extent to which RNF11 affects dopamine signaling in adults is unknown.

Here, I have studied acetylcholine-stimulated dopamine release in adult Drosophila at different ages, including control Canton S flies and flies with ParkinRNAi or RNF11-RNAi mutations. Previously, acetylcholine-stimulated dopamine release was measured in Drosophila larval VNC, but here acetylcholinestimulated dopamine release in adult Drosophila brains is measured for the first time. The measurements are made with FSCV at CFMEs and the small size of CFME allowed measurements of dopamine in the central complex region of Drosophila brain. The changes in tissue content of dopamine with age were also investigated in these lines. There is a significant difference in dopamine tissue content in adult Drosophila with the RNF11-RNAi mutation as Drosophila age. I 
also found that dopamine releasable pool replenishes faster in aging control, but not in the Parkin or RNF11 knockdown lines. There are conflicting evidence on the effects of different Parkinson's disease mutations in dopamine levels in Drosophila brains, ${ }^{24,25,32}$ and the results here indicate that Parkin-RNAi or RNF11-RNAi mutations do affect how fast the releasable pool of dopamine restores in old-age flies.

\subsection{Results}

\subsubsection{Acetylcholine-stimulated dopamine release in Drosophila melanogaster adult brain}

Acetylcholine-stimulated dopamine release was discovered in Drosophila larval VNC, and here we measured stimulated release in adult Drosophila brains for the first time. A CFME was placed $50 \mu \mathrm{m}$ deep into the center of the brain to target the central complex (Fig 3.1A). A pipet filled with $5 \mathrm{mM}$ acetylcholine was placed about $10 \mu \mathrm{m}$ from the CFME. When acetylcholine was puffed into the brain, a current response for dopamine was measured with FSCV (Fig 3.1B). Acetylcholine is not electroactive and is not measured. A horizontal slice of the color plot was taken to see how dopamine concentration changes with time (Fig 3.1B center). A vertical slice of the color plot was taken at maximal current, which shows the CV has the fingerprint of dopamine (Fig 3.1B inset). 


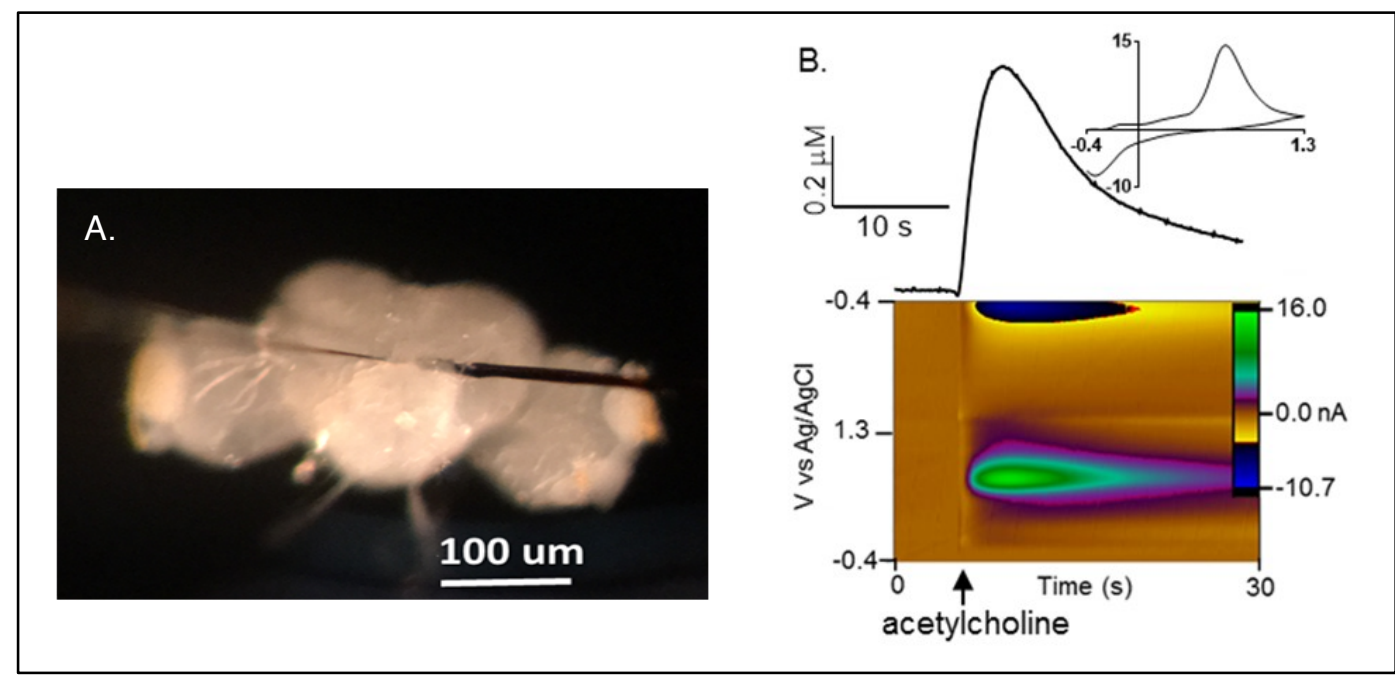

Figure 3.1. Acetylcholine-stimulated dopamine in the central complex region of Drosophila melanogaster brain. (A) Image of Drosophila brain with electrode coming from left and placed on the central complex. The image also shows stimulating pipet coming from the right. (B) The false color plot (bottom) and concentration vs. time plot (center) show how current changes over time. When 10 pmol acetylcholine is puffed on at $\mathbf{5}$ mins, there is a signal. The green oval in the center is oxidation current. When a vertical slice of the color plot is taken at peak current time a CV is obtained (inset). The electrochemical fingerprint of the CV shows that the current is due to dopamine.

The concentration of dopamine released with different amounts of acetylcholine stimulation was measured (Fig. 3.2). There is a significant effect of the amount of acetylcholine stimulation on evoked dopamine concentration (oneway ANOVA, $p=0.0004, n=6)$. The concentration of dopamine increases with increasing acetylcholine stimulation, and plateaus at 10 pmol acetylcholine after which it is not significantly different up to 40 pmol (one-way ANOVA Tukey's multiple comparisons test, $\mathrm{p}<0.05)$. Therefore, to measure maximal release 10 pmol acetylcholine stimulation was used in adult Drosophila brains. 


\section{Ach-stimulated dopamine}

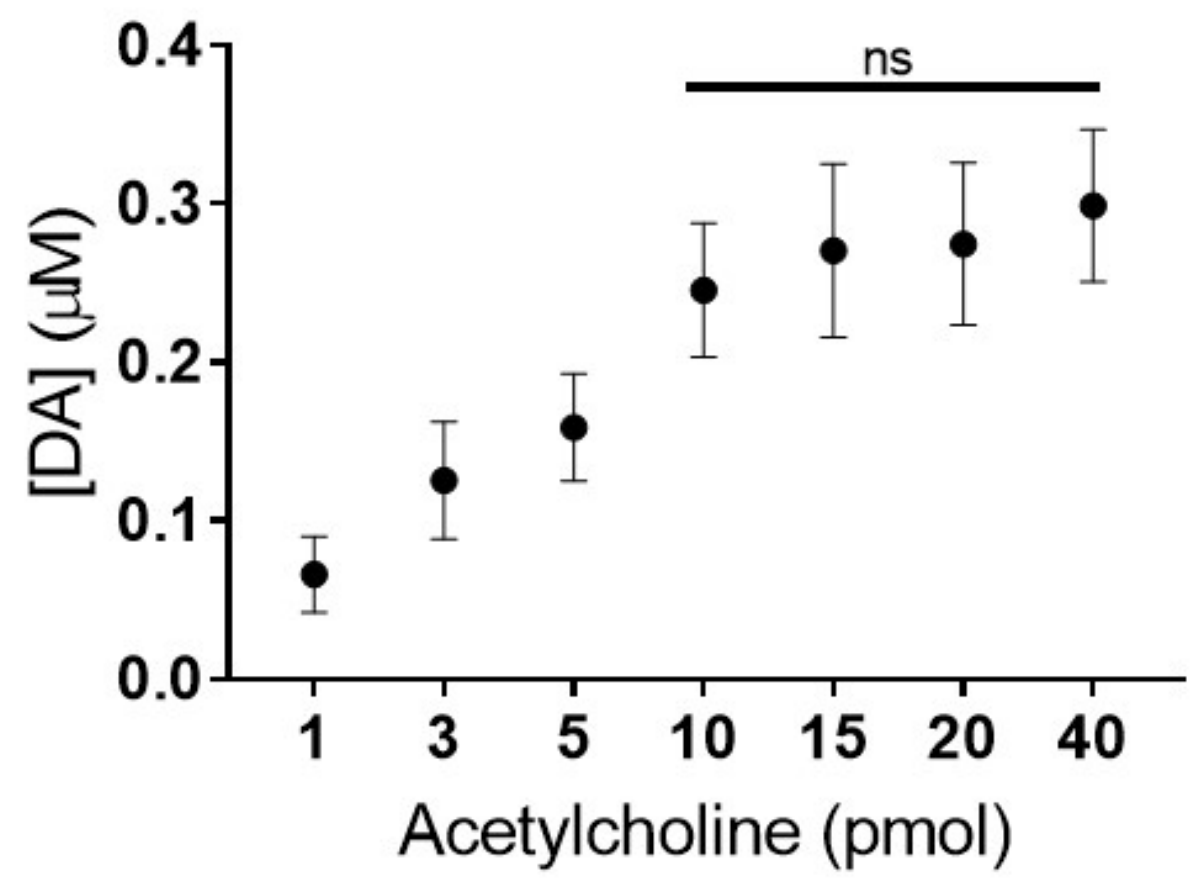

Figure 3.2. Dopamine release with increasing acetylcholine stimulation. There is a significant effect of the amount of acetylcholine stimulation as the concentration of evoked dopamine increases with increasing acetylcholine stimulation (one-way ANOVA, $p=0.0004, n=6$ ). The response plateaus at $10 \mathrm{pmol}$ as there is no significant increased in evoked dopamine concentration at higher than10 pmol stimulation (one-way ANOVA, Tukey's multiple comparisons test, $p<0.05$ ).

\subsubsection{Stimulated dopamine and dopamine tissue content with age}

Levels of dopamine synthesis enzyme $(\mathrm{TH})$ in the striatum decrease with age in humans and correlate with dopaminergic cells loss in the substantia nigra, where dopamine is synthesized. ${ }^{33,34}$ We hypothesized that similar to human brains, Drosophila CNS will have a significant reduction in dopamine with age. We studied stimulated dopamine release and dopamine tissue content at different ages in adult Drosophila. 
Acetylcholine stimulated dopamine release in the central complex region was studied at three different ages - young adult (1-5 day old), mid-age adult (20-25 day) and old adult (40-45 day). Figure 3.3 A-C show the color plots, concentration vs. time plots, and the CVs for stimulated release at different ages. The concentration of dopamine released with $10 \mathrm{pmol}$ acetylcholine stimulation was not significantly different between the age groups (one-way ANOVA, $p=$ 0.6474, $n=4-6$ ) (Fig. 3.3D). In contrast to our hypothesis, the release was slightly higher as Drosophila aged, although the result was not significantly different. Dopamine tissue content in the CNS was also not significantly different at the different ages (one-way ANOVA, $p=0.0758, \mathrm{n}=5-8$ ) (Fig. 3.3E). 


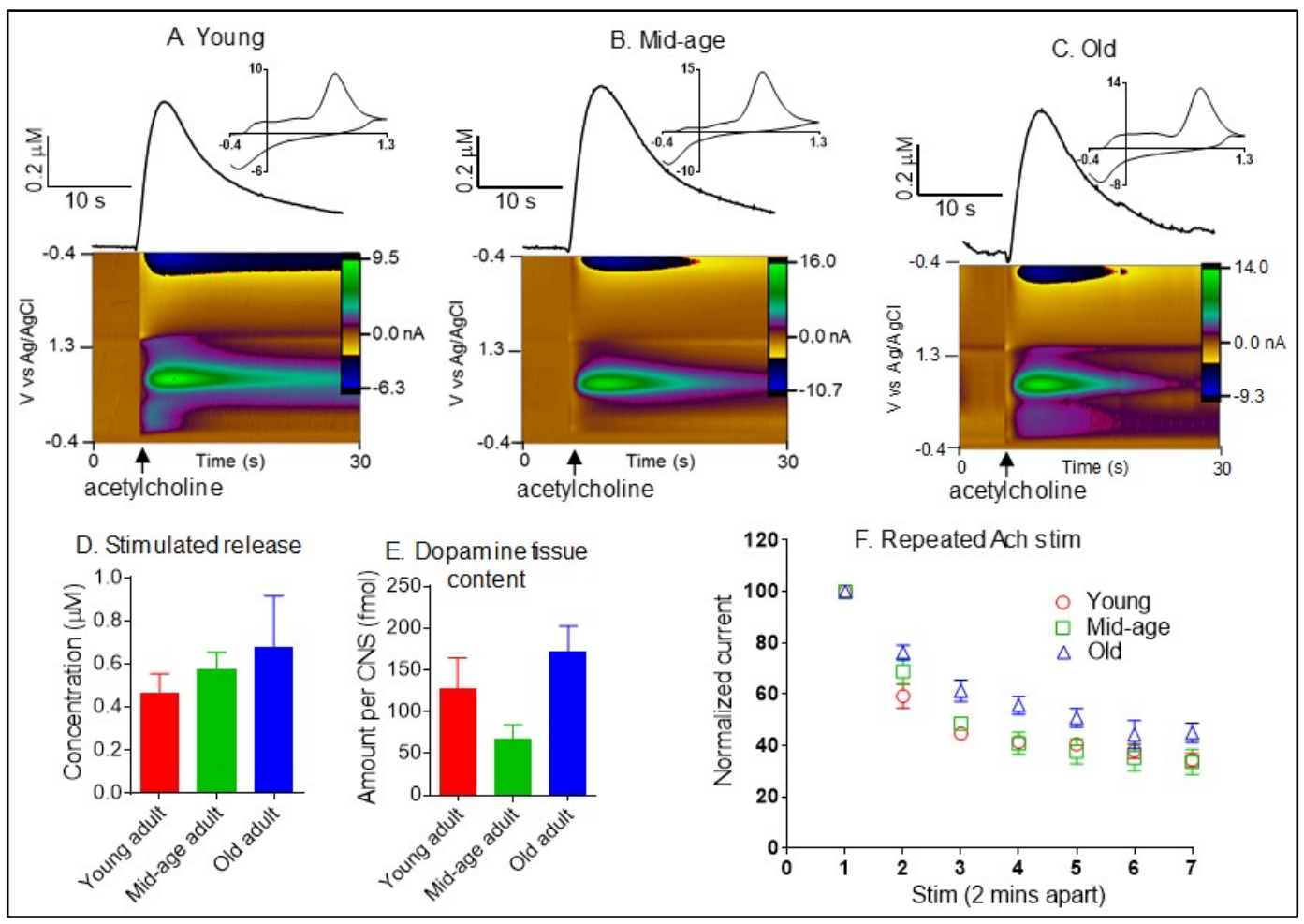

Figure 3.3. Acetylcholine-stimulated dopamine release in Drosophila melanogaster brain and tissue content in the CNS. Example plots for stimulated release in (A) young (1-5 day), (B) mid-age (20-25 day), and (C) old age adults (40-45 day). (D) Average concentrations of evoked dopamine for the different age groups. Although there is a slight increase in evoked dopamine with age, the difference is not significant (one-way ANOVA, $p=0.6474, n=4-6$ ). (E) The tissue content of dopamine in Drosophila melanogaster CNS at different ages. There was no significant effect of age on the tissue content of aging flies (one-way ANOVA, $p=0.0758, n=5-8$ ). (F) Acetylcholine stimulation was repeated at $2 \mathrm{~min}$ intervals and the responses were normalized to the first evoked current. With repeated stimulations, there are main effects of both age and stimulation number on evoked dopamine release but no interaction (2-way ANOVA, $p<0.05$ for age and $p<0.0001$ for stimulation number, interaction $p=0.1314$ ).

To see if there are any changes in dopamine dynamics with age, acetylcholine-stimulated dopamine release was measured at 2 min interstimulation times. Newly synthesized dopamine and dopamine reuptake through transporters contribute to the releasable pool of dopamine..$^{35}$ It takes longer than 2 minutes for dopamine releasable pool to replenish in Drosophila larva. ${ }^{36}$ On a short time scale, majority the releasable pool is maintained by reuptake, not synthesis. ${ }^{36}$ Therefore the effect of reuptake on dopamine release with multiple 
stimulations was studied by stimulating every two minutes. To correct for sample variability, the data were normalized to the peak concentration of the first stimulation (Fig. 3.3F). With repeated stimulations, there was a significant effect of age and a significant effect of stimulation number on evoked dopamine but no significant interaction (two-way ANOVA, $p<0.05, \mathrm{n}=4-6$ ). Evoked dopamine is significantly lower with repeated stimulations, and response for old adult does not decrease as fast as it does for the mid-age adults. Surprisingly, the older flies are better able to maintain their dopamine release over multiple stimulations.

\subsubsection{Stimulated dopamine and dopamine tissue content with age in Parkin knockdown}

As mentioned above, there is a significant loss of dopaminergic cells during aging in humans. In patients with Parkinson's disease, the loss of dopaminergic cells in the substantial nigra is significantly higher than the control population. ${ }^{37}$ We studied changes in evoked dopamine release with age in Drosophila with a knockdown of the Parkin gene with RNAi. Figure 3.4 A-C show example traces of concentration vs. time plot and CV at different ages for ParkinRNAi. Previous work in our lab showed no difference in evoked dopamine release in Parkin knockout Drosophila larvae compared to control (data not shown), but since the disease is age related, we studied effects of Parkin knockout in adult Drosophila as they age (Fig. 3.4D). 


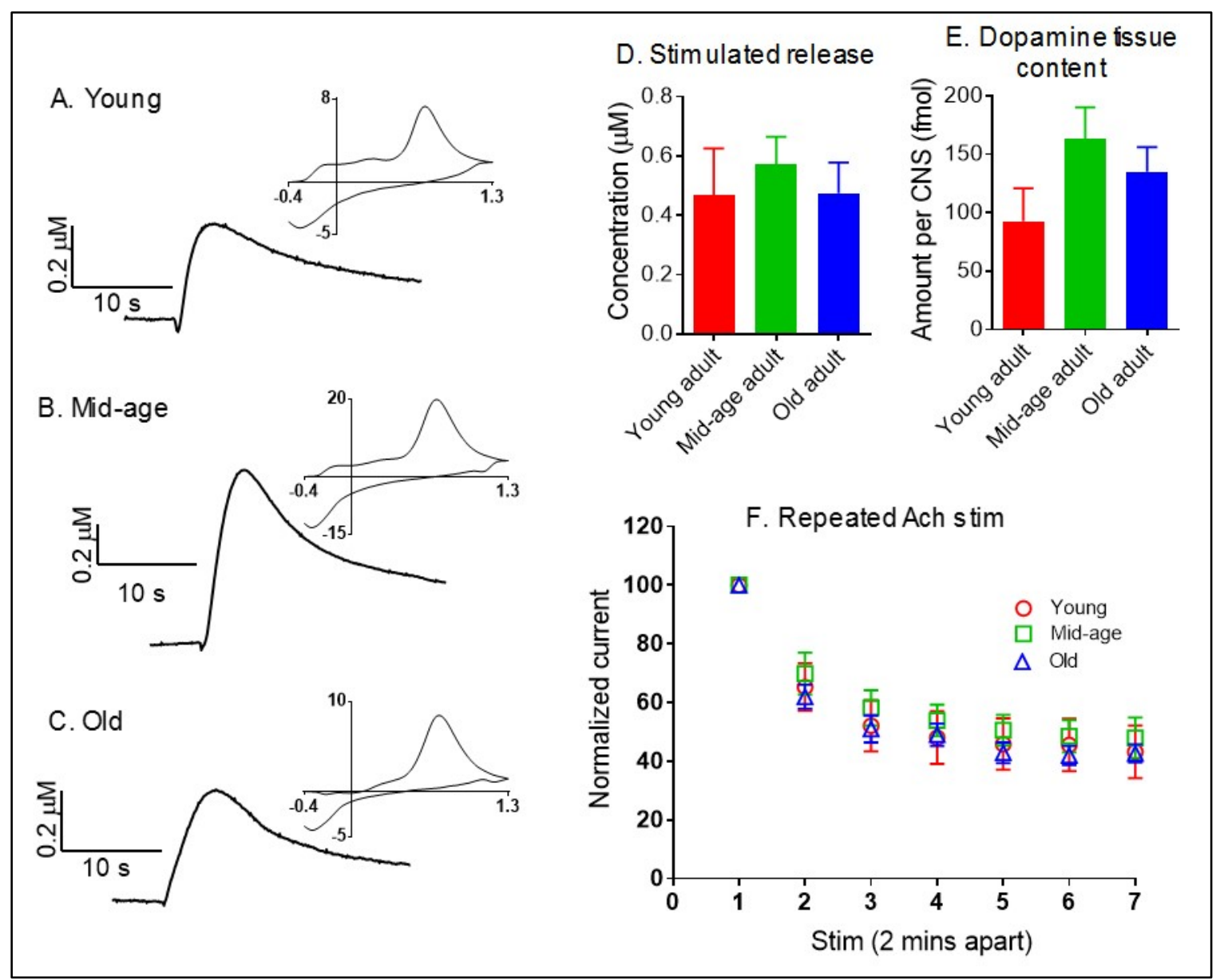

Figure 3.4. Acetylcholine-stimulated dopamine release in Drosophila melanogaster brain and tissue content in the CNS in Parkin-RNAi. Example plots for stimulated release in (A) young (1-5 day), (B) mid-age (20-25 day), and (C) old adults (40-45 day). (D) Average concentrations of evoked dopamine for the different age groups. There is no significant difference in stimulated release with age (oneway ANOVA, $p=0.7977, n=5-6)$. (E) The tissue content of dopamine in Parkin-RNAi Drosophila melanogaster CNS at different ages. There was no significant effect of age on the tissue content of aging flies (one-way ANOVA, $p=0.7543, n=6-7$ ). (F) Acetylcholine stimulation was repeated at $2 \mathrm{~min}$ intervals and the responses were normalized to the first evoked current. With repeated stimulations, there was no significant effect of age, but a significant effect of stimulation number on evoked dopamine release (2-way ANOVA, $p=0.7265$ for age and $p<0.0001$ for stimulation number).

Acetylcholine stimulated release in the central complex of Parkin knockdown Drosophila was not significantly different at different ages (one-way ANOVA, $p=0.7977, \mathrm{n}=5-6)$. The tissue content of dopamine in the CNS of Drosophila was also not significantly different with age in the Parkin knockdown Drosophila (one-way ANOVA, $p=0.7543, \mathrm{n}=6-7$ ) (Fig. 3.4E). Thus, the Parkin 
flies were not more quickly depleted of dopamine. When acetylcholine stimulation was repeated every 2 minutes, and data were normalized, there was a significant effect of stimulation number (two-way ANOVA, $p<0.0001$ ) but no effect of age (two-way ANOVA, $p=0.7265$ ) (Fig. 3.4F). Thus, the Parkin-RNAi flies did not have a significant effect of age as the control flies did.

\subsubsection{Stimulated dopamine and dopamine tissue content with age in RNF11 knockdown}

Downregulating RNF11 has been proposed as a treatment for PD as we found that RNF11 knockdown in Drosophila increases dopamine release and clearance in larval VNC. ${ }^{31}$ However, we have not studied the effects of RNF11 knockdown in adult Drosophila. Since Parkinson is an age related disease, we studied stimulated dopamine release in the central complex region of the brain of aging adult Drosophila with RNF11 knockdown. Figure 3.5A-C show example traces of the release at different ages in RNF11-RNAi flies. There was no significant effect of age on stimulated dopamine concentration in RNF11 knockdown Drosophila (one-way ANOVA, $p=0.4422, \mathrm{n}=5-6$ ) (Fig. 3.5D). There was, however, a significant effect of age on the tissue content of dopamine in the CNS of aging RNF11 knockdown Drosophila (one-way ANOVA, $p=0.0284, \mathrm{n}=$ 5-6) (Fig. 3.5E). Old flies had more dopamine with the RNF11 mutation. 


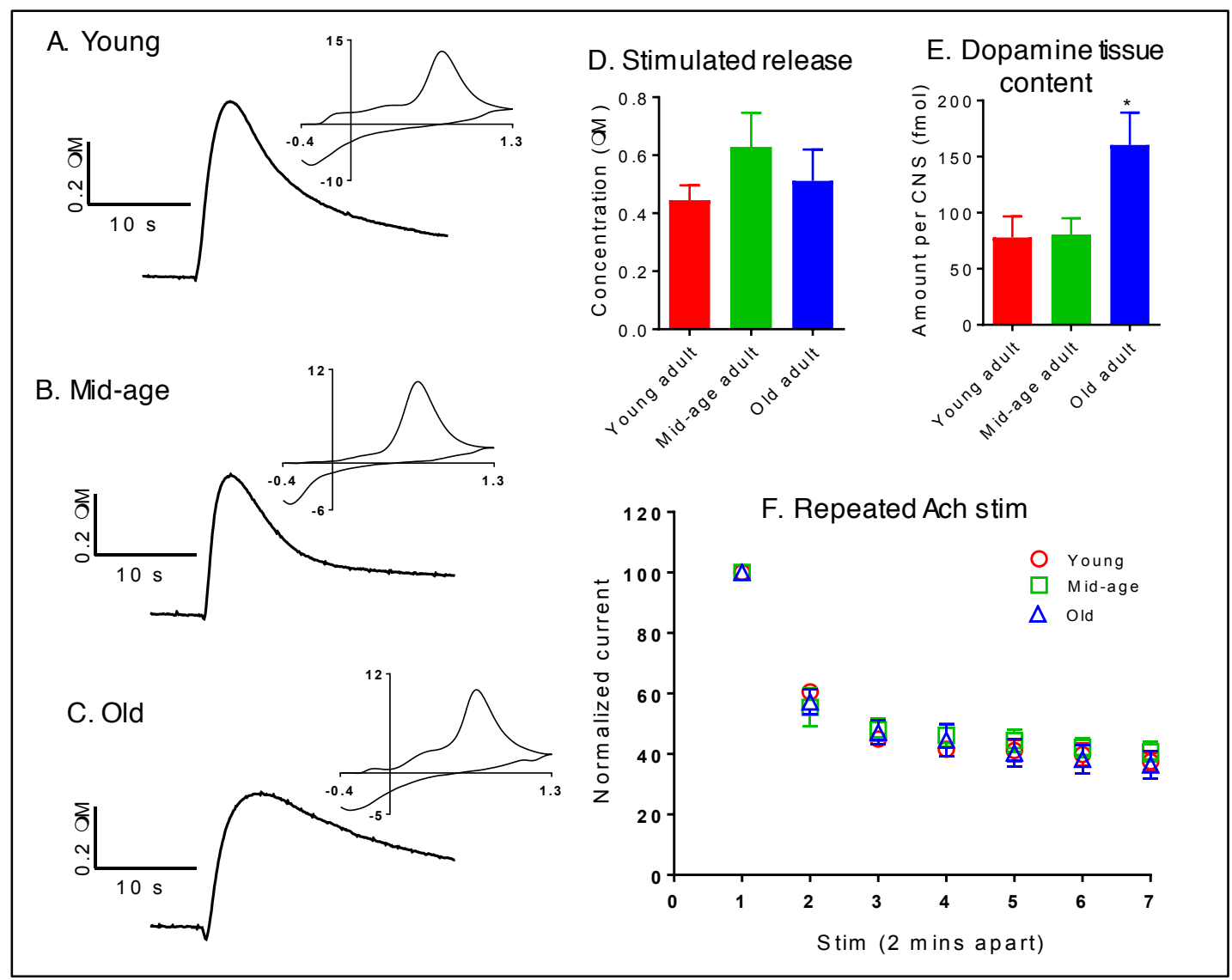

Figure 3.5. Acetylcholine-stimulated dopamine release in Drosophila melanogaster brain and tissue content in the CNS in RNF11-RNAi. Example plots for stimulated release in (A) young (1-5 day), (B) mid-age (20-25 day), and (C) old adults (40-45 day). (D) Average concentrations of evoked dopamine for the different age groups. There is no significant difference in stimulated release with age (oneway ANOVA, $p=0.4422, n=5-6)$. (E) The tissue content of dopamine in Parkin-RNAi Drosophila melanogaster CNS at different ages. There was a significant effect of age on the tissue content of aging flies (one-way ANOVA, $p=0.0284, n=5-6$ ). (F) Acetylcholine stimulation was repeated at $2 \mathrm{~min}$ intervals and the responses were normalized to the first evoked current. With repeated stimulations, there was no significant effect of age, but a significant effect of stimulation number on evoked dopamine release (2-way ANOVA, $p=0.8962$ for age and $p<0.0001$ for stimulation number).

Evoked dopamine current was studied with repeated stimulations at 2 minutes inter-stimulation times at different ages. The current was normalized to the first dopamine oxidation peak current (Fig. 3.5F). Two-way ANOVA showed significant effect of stimulation number (two-way ANOVA, $p<0.0001, \mathrm{n}=5-6$ ) but no effect of age on the release (two-way ANOVA, $p=0.8962, n=5-6$ ). 
Similar to Parkin-RNAi, there was no significant effect of age observed in RNF11RNAi with repeated stimulations. Therefore, only the control flies show a significantly higher release at old age when the stimulations were repeated at short time intervals and this effect is lost in the mutant flies.

\subsubsection{Comparison of release with repeated stimulations in different lines}

The 2-minute interval repeated stimulations data were compared among the different lines for the different age groups (Fig. 3.6). For all age groups, there was a significant effect of stimulation number but no significant effect of the genotype (two-way ANOVA, $p<0.0001$ for stimulation number and $p>0.1$ for genotype). For old-age control adults (Fig. 3.6A), the initial drop in stimulated current is not as great as for Parkin and RNF11 knockdowns (Figs. 3.6B and C), but reaches similar levels on further stimulations. When only the first three stimulations are compared, there is a significant effect of genotype as the evoked current in the control line does not decay as much as in other lines (two-way ANOVA, $p<0.05)$. Therefore, for old-age control there is a significantly less decay in evoked current when compared to mid-age group, and also a significantly less decay in the three initial stimulations when compared to other genotypes. 


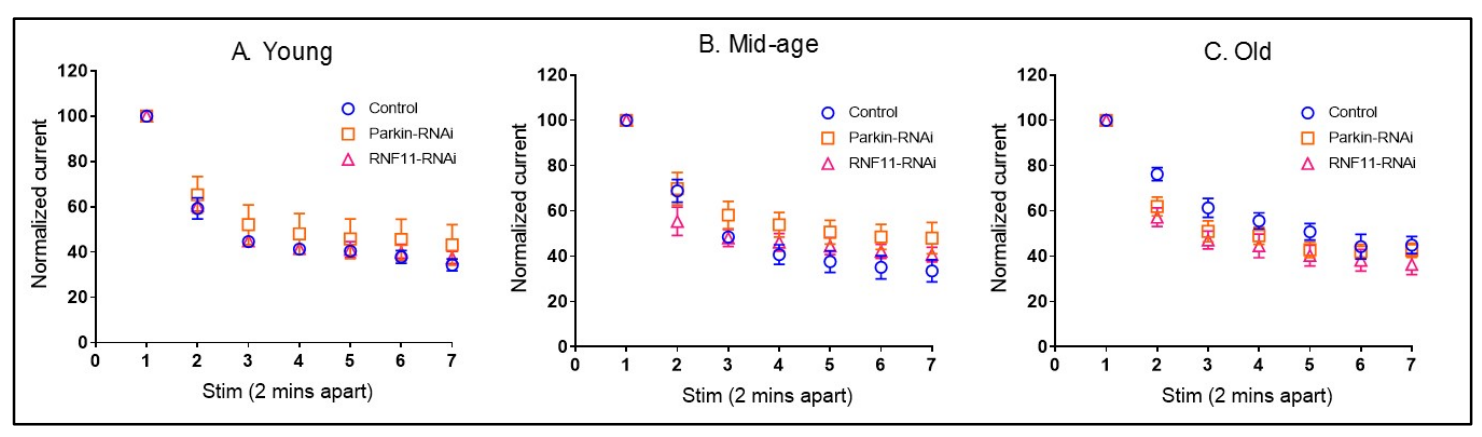

Figure 3.6. Acetylcholine-stimulated release in control, Parkin-RNAi, and RNF11-RNAi Drosophila repeated every 2 minutes and normalized to the first stimulation peak current. (A) Effect of genotype on young (1-5 day) flies. There was no significant effect of genotype, but a significant effect of stimulation number (2-way ANOVA, $p=0.6438$ for genotype and $p<0.0001$ for stimulation number). (B) Effect of genotype on mid-age (20-25 day) flies. There was again no significant effect of genotype, but a significant effect of stimulation number (2-way ANOVA, $p=0.2216$ for genotype and $p<0.0001$ for stimulation number). (C) Effect of genotype on old (40-45 day) flies. There was no significant effect of genotype, but a significant effect of stimulation number (2-way ANOVA, $p=$ 0.1352 for genotype and $p<0.0001$ for stimulation number).

\subsection{Discussion}

Parkinson's disease is a neurodegenerative disorder associated with aging that is characterized by a substantial loss of dopaminergic cells in the substantia nigra. Drosophila melanogaster is a popular model organism to study the disease, and there are many lines that model Parkinson's disease. Here, we studied effect of aging on stimulated dopamine release and tissue content in control flies, and flies with Parkin or RNF11 knockdown. We found no significant change in the tissue content of dopamine in the CNS of Drosophila as they age normally or with Parkin knockdown, but there is a significant increase in the tissue content in old RNF11-RNAi flies. We found no significant effect of age on dopamine release with acetylcholine stimulation, but when stimulations are repeated at 2 min intervals there was a significant effect of age in control flies, but not in the knockdown lines. Parkin knockout mutation, therefore, does not 
affect dopamine levels in the CNS, but affects dopamine dynamics in the central complex of the brain with age in Drosophila adults. When Parkinson's disease is diagnosed in humans, an approximately $80 \%$ of dopaminergic cells have already died. The higher levels of dopamine in RNF11-RNAi seen here suggests that the downregulation of RNF11 can be a compensatory mechanism to keep dopamine levels up in living cells when other cells degenerate. We show here that Drosophila can provide an understanding of fundamental changes in dopamine signaling that occurs with aging with Parkinson's disease.

\subsubsection{Acetylcholine stimulated dopamine release in Drosophila melanogaster adult brain}

Acetylcholine stimulated dopamine release in Drosophila larvae was recently discovered in our lab (Chapter 2). Here we measured stimulated dopamine in the central complex region of adult Drosophila brain. The central complex consists of five structures namely protocerebral bridge, fan-shaped body, ellipsoid body, paired noduli, and paired lateral accessory lobes. ${ }^{38}$ The ellipsoid body and fan shaped body are the largest regions and thus it is most likely our electrode is implanted there. The central complex is rich in dopaminergic projections and the structure is involved in motor functions in Drosophila. ${ }^{39-42}$ Also, the central complex and human basal ganglia, which contains the substantia nigra are considered evolutionarily conserved and share similar neurochemistry and behavioral outcomes. ${ }^{38}$ The substantia nigra is the structure where a substantial loss of dopaminergic cells occurs in Parkinson's 
disease. ${ }^{37}$ The concentration of evoked dopamine in the central complex was dependent on the amount of acetylcholine stimulation with 10 pmol stimulation resulting in maximal response. Thus $10 \mathrm{pmol}$ acetylcholine stimulation was used to study dopamine release in aging flies and in flies with Parkinson's disease mutations.

\subsubsection{Stimulated dopamine release and dopamine tissue content during aging in Drosophila}

The goal of this project was to study age dependent dopamine changes in Drosophila with Parkinson's disease mutations, and we first studied stimulated dopamine and dopamine tissue content in the CNS of control flies. Previous studies had shown that whole body dopamine levels of Drosophila decrease with age ${ }^{43}$ Another study in Drosophila also saw a decrease of $\mathrm{TH}$ labeling with age in the PPL2ab region of dopaminergic cells. ${ }^{26}$ Here we observed no significant difference with age in either the concentration of acetylcholine-stimulated dopamine release or the tissue content of dopamine in the CNS of adult Drosophila. The result agree with other reports where GFP and antisera labeled TH had no significant decrease in Drosophila brains with age. ${ }^{44}$ Although the whole body dopamine and subsets of dopaminergic cells decrease with age in Drosophila, our results and others show that there is no significant decrease of overall dopamine levels in the CNS or stimulated dopamine release in the central complex region. Drosophila exoskeleton contains dopamine so whole body measurements do not reflect the neurochemical functions. Our results show that 
there is no deficit in dopamine function with age in the nervous system in normal Drosophila.

In humans there is a significant reduction of striatal TH activity and a decrease in the number of substantia nigra cells between the age of 20 to 90 years. ${ }^{33,34}$ The rate of dopaminergic cell death in the substantia nigra of the brain is $2.1-6.9 \%$ per decade in different parts of the substantia nigra in a control population. ${ }^{37}$ There is also an age-related decline of dopamine transporters in various parts of the brain. ${ }^{45}$ In Drosophila, there are reports of a decline of subsets of dopaminergic neurons, but our results show that it does not affect dopamine release in the central complex. Future studies should therefore investigate dopamine content and stimulated release in different regions of the Drosophila CNS to see if there is a region specific decline of dopamine content or release with age. We could also do staining of cells to verify the extent to which TH is decreased in our particular fly lines and the extent of dopaminergic cell death with age. With this information, we could better correlate cell death and neurochemistry in the future.

When acetylcholine-stimulation was repeated at 2 minutes interstimulation times, there was a significant effect of age and of stimulation number. The effect of stimulation number shows that dopamine releasable pool does not replenish when acetylcholine stimulation is repeated every 2 minutes. The result is similar to ATP stimulated release in transgenic Drosophila larvae expressing $\mathrm{P} 2 \mathrm{X}_{2}$ channels in dopaminergic neurons. ${ }^{36}$ ATP-stimulated release went down to 
about $60 \%$ in the transgenic larvae while acetylcholine-stimulated response went down to $34-45 \%$ in the adults on the $7^{\text {th }}$ stimulation. The difference in decrease can be due to the difference in the stimulation method, life stage or region of the CNS tested. Future studies can therefore investigate tissue content in specific brain regions of adults and also study stimulated release in other regions of the adult brains to see if there are changes with aging. The significant effect of age on repeated stimulations with old-age having a less decrease compared to midage flies shows that the old flies maintain dopamine signaling better than younger flies when stimulations are repeated at 2 min intervals. Dopamine uptake is therefore maintained better in the old flies, and future research will investigate how uptake parameters change with aging flies.

\subsubsection{Stimulated dopamine release and dopamine tissue content in Parkin knockdown Drosophila}

Parkinson's disease model Drosophila show a significant loss of subsets

of dopaminergic neurons and a decrease in TH expression. ${ }^{14,16,46}$ We measured dopamine tissue content and stimulated release to see if Parkin knockout mutations have an effect on dopamine levels or tissue content. Since Parkinson's is an age related disease and expression of mutant human Parkin in Drosophila causes an age-dependent loss of dopamine neurons ${ }^{45}$ we studied the effect of aging in dopamine signaling and content in Parkin knockout flies. There was no significant difference in either the CNS tissue content or stimulated concentration of dopamine in the central complex in aging Parkin knockdown flies. There was 
also no significant difference of age when acetylcholine-stimulation was repeated at 2 minutes interval.

Compared to the control population, Parkinson's disease patients have a significantly higher dopaminergic cell loss of $45 \%$ in the substantia nigra during the first decade of a study. ${ }^{37}$ The percent cell loss was different in different parts of the substantia nigra. We found that in Parkin knockdown Drosophila, there is no significant decrease in dopamine content in the CNS or in stimulated dopamine release as they age. Past studies have given conflicting evidence on the effect of Parkin knockdown on dopaminergic cells. Pesah et. al tested Parkin mutant Drosophila for dopaminergic cell loss in the dorsomedial clusters and found no significant difference in $\mathrm{TH}$ expression or any defect in the appearance of dopamine cell bodies in 3 week old Parkin mutant flies compared to control. ${ }^{24}$ They did however observe a loss of flight muscles and suggested that Parkin may play an important role in cells with high energy demand. Another study using Parkin knockdown with RNAi also reported no difference in TH expression in posterior protocerebral dopaminergic neuronal clusters compared to control. ${ }^{25}$ Yet another study found that there is a shrinkage of dopaminergic cells and reduced TH expression in dorsomedial dopaminergic cells of aged Parkin mutant flies compared to control, but other dopaminergic cells tested did not appear different in the mutant line. ${ }^{32}$

Therefore, it seems that Parkin mutants show variable effects on dopaminergic cells and there may be brain regions that are prone due to the 
mutation while other regions that are not. Another possibility is that Parkin mutation alone is not enough for causing dopaminergic cell death. In humans, the disease is often linked to both genetic and environmental causes, and disease mutations do not always lead to Parkinson's disease. ${ }^{47}$ Similarly in Drosophila, Parkin knockdown along with a toxin exposure caused dopaminergic cell death, but the mutation alone did not affect the cells. ${ }^{25}$ It is therefore possible that some mutations in Parkin are more likely than others to cause the disease and that some mutations only increase the susceptibility to the disease. Two independent studies report that expression of mutant Parkin associated with human familial Parkinson's disease in Drosophila causes dopaminergic cell loss in neurons. ${ }^{46}$ Future research should therefore examine Drosophila lines that have shown loss of dopaminergic cells and also focus on regions where cell loss has been observed.

\subsubsection{Stimulated dopamine release and dopamine tissue content in RNF11 knockdown Drosophila}

RNF11 is a negative regulator of the NF-kappa B pathway, and chronic activation of the pathway has been linked to neurodegeneration in Parkinson's disease. RNF11 knockdown increased protection of dopaminergic cells in vitro and in rat 6-OHDA Parkinson's disease models. ${ }^{30}$ The neuroprotection was suggested to be because of increased activity of NF-kappa B. We also found that RNF11 knockdown in Drosophila increases dopamine release and clearance in larval VNC. ${ }^{31}$ Here, we studied the effects of RNF11 knockdown in aging adult 
Drosophila. There was no significant difference in stimulated dopamine release with age but a significant difference in the tissue content with age. The old flies with RNF11 knockdown had significantly higher dopamine levels in the CNS than younger flies - the effect that was not observed in either control or Parkin-RNAi flies. Therefore, RNF11 knockdown acts to keep a high level of dopamine in the CNS. It is possible that the downregulation of RNF11 in surviving cells of Parkinson's disease patients may be why symptoms only appear after up to $80 \%$ of cells have died as the total content of dopamine is maintained for a long time.

The stimulated dopamine result in adults is different than larvae, where RNF11-RNAi does have an effect on evoked concentration and the mutant line has a significantly higher evoked concentration than control. ${ }^{31}$ It is important to note than the stimulations used in these studies are different. The stimulation used in this study (10 pmol acetylcholine) gives maximal response in control adults, but the stimulation used in larvae $(2 \mathrm{~s}$ light stim: $60 \mathrm{~Hz}, 120$ pulses, $4 \mathrm{~ms}$ red light each pulse) does not give maximal response in control adults. It is therefore possible that we are overstimulating the cells in adults, which is preventing us to observe any increase in dopamine release in the RNF11-RNAi line. Future studies should therefore use a smaller stimulation in both control and $R N F 11-R N A i$ lines to see if there will be any significant difference in evoked current response in the two lines. When acetylcholine-stimulations were repeated at 2 min intervals in RNF11-RNAi adults, there was an effect of stimulation number, but not of age. This is different than in controls where there was a 
significantly less decrease at old age. RNF11-RNAi knockdown therefore affects the levels of dopamine with age and also how dopamine pool restores when there is a short interval between stimulations.

\subsubsection{Repeated stimulations compared in different Drosophila lines}

When the data for control vs. Parkinson's disease model flies were compared for different age groups, there was a significant effect of genotype when only the first three stimulations were compared. The old-age control group had a significantly lower decay during the first three stimulations than the Parkin or RNF11 knockdowns. When all seven stimulations were compared, however, there was no effect of genotype on the old-age group. For young and mid-age groups, there was no significant effect of age on evoked current with repeated stimulations. The results show that with normal aging, the dopamine signaling is maintained better than aging with Parkinson's disease mutations.

\subsection{Conclusions}

We showed here that dopamine tissue content and stimulated dopamine release do not decrease with age in Drosophila. The tissue content is slightly but not significantly higher in older adult controls, and significantly higher in old age $R N F 11-R N A i$ adults. When acetylcholine-stimulations were repeated at 2 min intervals, there was a significant effect of age on control flies but not in the Parkin-RNAi or RNF11-RNAi. In the control flies there was less decrease of stimulated release for old flies, but the effect was not observed in Parkin or 
RNF11 knockdowns. This suggests that as control flies age they have a faster replenishment of releasable dopamine pool compared to the Parkinson's disease mutants. No significant differences in dopamine content or release with aging in Parkin mutants suggests that the overall CNS content of dopamine is not affected in these mutants.

\subsection{Future Studies}

Drosophila melanogaster lines used to model Parkinson's disease in this study had a knockdown of Parkin or RNF11-RNAigenes in the dopaminergic cells. Knockdown with RNAi typically decreases the expression by about $50 \%$, but is not a full knockout. Past research on Parkin knockouts provides evidence that not all dopaminergic cells are affected in these flies during aging. ${ }^{24,25,32}$ Thus, it is important in the future to study additional brain regions as well. Models of the disease where mutant forms of human Parkin genes are expressed in Drosophila show degeneration of dopaminergic cells, and may be a better model to study the disease in the future. ${ }^{4548}$ Future studies should also focus on the PPL and PAM regions, where dopaminergic cell degeneration has been observed in some Parkin mutants. ${ }^{26,48}$

\subsection{Experimental Section}

\subsubsection{Chemicals}

All chemicals were purchased from Sigma-Aldrich (St. Louis, MO), and solutions were prepared in Milli Q water (Millipore, Billerica, MA) unless noted 
otherwise. Electrode calibrations were performed in phosphate buffer solution (PBS; $131.25 \mathrm{mM} \mathrm{NaCl}, 3.0 \mathrm{mM} \mathrm{KCl}, 10.0 \mathrm{mM} \mathrm{NaH}_{2} \mathrm{PO}_{4}, 1.2 \mathrm{mM} \mathrm{MgCl}_{2}, 2.0 \mathrm{mM}$ $\mathrm{Na}_{2} \mathrm{SO}_{4}$, and $1.2 \mathrm{mM} \mathrm{CaCl}_{2}$ ) with $\mathrm{pH}$ adjusted to 7.4 , which was made once a month and stored at $4{ }^{\circ} \mathrm{C}$. To make the dissection buffer, $11.1 \mathrm{mM}$ glucose and $5.3 \mathrm{mM}$ trehalose were added to the PBS buffer on the day of the experiment. A $10 \mathrm{mM}$ stock solution of dopamine was prepared in $0.1 \mathrm{M} \mathrm{HClO}_{4}$ once a month and stored at $4{ }^{\circ} \mathrm{C}$. Dilute dopamine solution for electrode calibration was prepared in PBS buffer the day of the experiment. Acetylcholine chloride (5 mM) solution was prepared in PBS.

\subsubsection{Drosophila dissection}

Drosophila melanogaster strains were obtained from Subhabrata Sanyal at Biogen, Inc.: GFP expression driven by GAL4 on dopaminergic neurons ( $\mathrm{TH}$ GAL4; UAS-GFP); UAS-RNAi on Parkin (UAS-Park-RNAi), and UAS-RNAi on RNF11 (UAS-RNF11-RNAI). Controls were TH-GAL4; UAS-GFP. Drosophila melanogaster stocks were maintained and crossed as described before. ${ }^{49}$ For stimulated release experiments, the adult brains were dissected out following procedures described before, but here Drosophila were anesthetized on ice instead of carbon dioxide. ${ }^{50}$ The dissected brain was transferred and plated to a petri dish with $3 \mathrm{~mL}$ dissection buffer.

For tissue content measurements, the whole CNS were dissected out in the dissection buffer following previously described methods with some 
modifications. ${ }^{51}$ The fly is held with tweezers on the abdomen and placed on a Sylgard-lined dish with buffer in it. A metal wire is used to pin the fly in the Sylgard through the abdomen. Next, the wings and legs are removed, and holding between the compound eye and the proboscis, the proboscis is also pulled out. The large trachea within the head capsule is removed. Next, the ventral part of the thorax is torn on both sides and the cuticle is removed. Tissues surrounding the VNC are cleaned out, and the VNC is carefully pulled while keeping it attached to the head. Next, grasping on the posterior corner of the head capsule with a set of tweezers, the compound eye is pulled out. The rest of the head capsule, compound eye and trachea are also removed taking care not the damage the brain to isolate the CNS.

\subsubsection{Drosophila homogenate preparation}

The tip of a gel-loading pipet is rotated on a flame to seal the tip. A fly CNS is transferred to the pipet tip with minimal dissection buffer. $2 \mu \mathrm{L}$ of $2 \mathrm{mM}$ perchloric acid is added to the sealed pipet tip. Next, a silver wire is used to break up the CNS and the sample vial is placed on a bath sonicator for 10 minutes. The sample vial is centrifuged at $14 \mathrm{rcf}$ for 3 minutes. The sample vial is then inverted over a centrifugal filter and the sample is centrifuged again for 3 minutes. The filtrate is transferred to a $500 \mu \mathrm{L}$ microcentrifuge tube.

\subsubsection{Instrumentation and data analysis}

\section{Stimulated dopamine release measurements}


Carbon-fiber microelectrode (CFME) was fabricated by previously described methods. ${ }^{52}$ Briefly, a $7 \mu \mathrm{m}$ diameter T-650 carbon fiber (Cytec Engineering Materials, West Patterson, $\mathrm{NJ}$ ) were pulled into a $1.2 \mathrm{~mm}$ o.d. glass capillary. The capillary was pulled with a vertical puller to make two electrodes. The electrode was trimmed so $50 \mu \mathrm{m}$ of carbon fiber was protruding. The tip of the electrode was epoxied to make a seal between the fiber and glass. The electrodes were calibrated with $1 \mu \mathrm{M}$ dopamine in a flow cell.

Drosophila brain was viewed under a stereomicroscope (Nikon Instruments Inc., Melville, NY) and electrode was inserted into the central complex. The potential was applied between the CFME and a $\mathrm{Ag} / \mathrm{AgCl}$ reference electrode in the bath. A picospritizing pipet was fabricated by pulling a $1.2 \mathrm{~mm}$ o.d. glass capillary, coating the tip with black Sharpie permanent marker for visualization of the tip. The pipet was trimmed and filled with acetylcholine. It was calibrated by measuring the diameter of liquid puffed on in oil at a set pressure and time. The pipet was also inserted into the brain about $10 \mu \mathrm{m}$ away from the CFME.

\section{Dopamine tissue content measurements}

The working electrode used for tissue content measurements with capillary electrophoresis was carbon-fiber disk electrode fabricated with $30 \mu \mathrm{m}$ diameter fiber (World Precision Instruments, Sarasota, FL). The CE with end column FSCV was built in house as described previously. ${ }^{53}$ Electrokinetic 
injection of the sample was performed by placing the end of the separation capillary into the microcentrifuge tube. Sample was injected by applying applying $+5 \mathrm{kV}$ for 15 secs. Separation of dopamine from other analytes was performed by applying $+9 \mathrm{kV}$ at the injection end of the capillary. The electrode was precalibrated with $100 \mathrm{nM}$ dopamine.

Data were collected with Waveneuro potentiostat (Pine Research Instrumentation, Durham, NC). HDCV software (a gift of Mark Wightman, University of North Carolina) was used for data collection and analysis. All statistics were performed in GraphPad Prism 6 (La Jolla, CA). Significance was measured with a $95 \%$ confidence interval. Graphs are shown as mean \pm standard error of the mean. 


\subsection{References}

[1] Hermanns, M. (2011) Weathering the storm: living with Parkinson's disease, $J$ Christ Nurs 28, 76-82; quiz 83-74.

[2] Morens, D. M., Davis, J. W., Grandinetti, A., Ross, G. W., Popper, J. S., and White, L. R. (1996) Epidemiologic observations on Parkinson's disease: Incidence and mortality in a prospective study of middle-aged men, Neurology 46, 1044-1050.

[3] Spatola, M., and Wider, C. (2014) Genetics of Parkinson's disease: the yield, Parkinsonism Relat Disord 20 Suppl 1, S35-38.

[4] Pan-Montojo, F., and Reichmann, H. (2014) Considerations on the role of environmental toxins in idiopathic Parkinson's disease pathophysiology, Transl Neurodegener 3, 10.

[5] Gasser, T. (1997) Advances in the genetics of movement disorders: Implications for molecular diagnosis, J Neurol 244, 341-348.

[6] Rakovic, A., Grunewald, A., Seibler, P., Ramirez, A., Kock, N., Orolicki, S., Lohmann, K., and Klein, C. (2010) Effect of endogenous mutant and wildtype PINK1 on Parkin in fibroblasts from Parkinson disease patients, Hum Mol Genet 19, 3124-3137.

[7] Polymeropoulos, M. H., Lavedan, C., Leroy, E., Ide, S. E., Dehejia, A., Dutra, A., Pike, B., Root, H., Rubenstein, J., Boyer, R., Stenroos, E. S., Chandrasekharappa, S., Athanassiadou, A., Papapetropoulos, T., Johnson, W. G., Lazzarini, A. M., Duvoisin, R. C., Dilorio, G., Golbe, L. I., and Nussbaum, R. L. (1997) Mutation in the alpha-synuclein gene identified in families with Parkinson's disease, Science 276, 2045-2047.

[8] Lucking, C. B., Durr, A., Bonifati, V., Vaughan, J., De Michele, G., Gasser, T., Harhangi, B. S., Meco, G., Denefle, P., Wood, N. W., Agid, Y., Brice, A., Suscep, E. C. G., and Stu, F. P. D. G. (2000) Association between earlyonset Parkinson's disease and mutations in the parkin gene, New Engl $J$ Med 342, 1560-1567.

[9] Zimprich, A., Biskup, S., Leitner, P., Lichtner, P., Farrer, M., Lincoln, S., Kachergus, J., Hulihan, M., Uitti, R. J., Calne, D. B., Stoessl, A. J., Pfeiffer, R. F., Patenge, N., Carbajal, I. C., Vieregge, P., Asmus, F., MullerMyhsok, B., Dickson, D. W., Meitinger, T., Strom, T. M., Wszolek, Z. K., 
and Gasser, T. (2004) Mutations in LRRK2 cause autosomal-dominant Parkinsonism with pleomorphic pathology, Neuron 44, 601-607.

[10] Forno, L. S. (1996) Neuropathology of Parkinson's disease, J Neuropath Exp Neur 55, 259-272.

[11] Bernheimer, H., Birkmayer, W., Hornykiewicz, O., Jellinger, K., and Seitelberger, F. (1973) Brain dopamine and the syndromes of Parkinson and Huntington. Clinical, morphological and neurochemical correlations, $J$ Neurol Sci 20, 415-455.

[12] de Lau, L. M., and Breteler, M. M. (2006) Epidemiology of Parkinson's disease, Lancet Neurol 5, 525-535.

[13] Sin, O., Michels, H., and Nollen, E. A. (2014) Genetic screens in Caenorhabditis elegans models for neurodegenerative diseases, Biochim Biophys Acta 1842, 1951-1959.

[14] Whitworth, A. J., Theodore, D. A., Greene, J. C., Benes, H., Wes, P. D., and Pallanck, L. J. (2005) Increased glutathione S-transferase activity rescues dopaminergic neuron loss in a Drosophila model of Parkinson's disease, $P$ Natl Acad Sci USA 102, 8024-8029.

[15] Feany, M. B., and Bender, W. W. (2000) A Drosophila model of Parkinson's disease, Nature 404, 394-398.

[16] Cha, G. H., Kim, S., Park, J., Lee, E., Kim, M., Lee, S. B., Kim, J. M., Chung, J., and Cho, K. S. (2005) Parkin negatively regulates JNK pathway in the dopaminergic neurons of Drosophila, Proc Natl Acad Sci U S A 102, 10345-10350.

[17] Scherzer, C. R., Jensen, R. V., Gullans, S. R., and Feany, M. B. (2003) Gene expression changes presage neurodegeneration in a Drosophila model of Parkinson's disease, Hum Mol Genet 12, 2457-2466.

[18] Whitworth, A. J. (2011) Drosophila Models of Parkinson's Disease, Adv Genet 73, 1-50.

[19] Yamamoto, S., and Seto, E. S. (2014) Dopamine Dynamics and Signaling in Drosophila: An Overview of Genes, Drugs and Behavioral Paradigms, Exp Anim Tokyo 63, 107-119. 
[20] Greene, J. C., Whitworth, A. J., Kuo, I., Andrews, L. A., Feany, M. B., and Pallanck, L. J. (2003) Mitochondrial pathology and apoptotic muscle degeneration in Drosophila parkin mutants, P Natl Acad Sci USA 100, 4078-4083.

[21] Winklhofer, K. F., and Haass, C. (2010) Mitochondrial dysfunction in Parkinson's disease, Bba-Mol Basis Dis 1802, 29-44.

[22] Cano-de-la-Cuerda, R., Perez-de-Heredia, M., Miangolarra-Page, J. C., Munoz-Hellin, E., and Fernandez-de-las-Penas, C. (2010) Is There Muscular Weakness in Parkinson's Disease?, Am J Phys Med Rehab 89, 70-76.

[23] Doherty, K. M., van de Warrenburg, B. P., Peralta, M. C., Silveira-Moriyama, L., Azulay, J. P., Gershanik, O. S., and Bloem, B. R. (2011) Postural deformities in Parkinson's disease, Lancet Neurol 10, 538-549.

[24] Pesah, Y., Pham, T., Burgess, H., Middlebrooks, B., Verstreken, P., Zhou, Y., Harding, M., Bellen, H., and Mardon, G. (2004) Drosophila parkin mutants have decreased mass and cell size and increased sensitivity to oxygen radical stress, Development 131, 2183-2194.

[25] Ortega-Arellano, H. F., Jimenez-Del-Rio, M., and Velez-Pardo, C. (2017) Minocycline protects, rescues and prevents knockdown transgenic parkin Drosophila against paraquat/iron toxicity: Implications for autosomic recessive juvenile parkinsonism, Neurotoxicology 60, 42-53.

[26] Kuo, S. Y., Wu, C. L., Hsieh, M. Y., Lin, C. T., Wen, R. K., Chen, L. C., Chen, Y. H., Yu, Y. W., Wang, H. D., Su, Y. J., Lin, C. J., Yang, C. Y., Guan, H. Y., Wang, P. Y., Lan, T. H., and Fu, T. F. (2015) PPL2ab neurons restore sexual responses in aged Drosophila males through dopamine, Nat Commun 6.

[27] Javoy-Agid, F., Hirsch, E. C., Dumas, S., Duyckaerts, C., Mallet, J., and Agid, Y. (1990) Decreased tyrosine hydroxylase messenger RNA in the surviving dopamine neurons of the substantia nigra in Parkinson's disease: an in situ hybridization study, Neuroscience 38, 245-253.

[28] Anderson, L. R., Betarbet, R., Gearing, M., Gulcher, J., Hicks, A. A., Stefansson, K., Lah, J. J., and Levey, A. I. (2007) PARK10 candidate RNF11 is expressed by vulnerable neurons and localizes to Lewy bodies in Parkinson disease brain, $J$ Neuropath Exp Neur 66, 955-964. 
[29] Pranski, E., Van Sanford, C. D., Dalal, N., Orr, A. L., Karmali, D., Cooper, D. S., Gearing, M., Lah, J. J., Levey, A. I., and Betarbet, R. (2013) NF-kappa $B$ activity is inversely correlated to RNF11 expression in Parkinson's disease, Neurosci Lett 547, 16-20.

[30] Pranski, E. L., Dalal, N. V., Van Sanford, C., Herskowitz, J. H., Gearing, M., Lazo, C., Miller, G. W., Lah, J. J., Levey, A. I., and Betarbet, R. S. (2013) RING finger protein 11 (RNF11) modulates susceptibility to 6-OHDAinduced nigral degeneration and behavioral deficits through NF-kappa B signaling in dopaminergic cells, Neurobiol Dis 54, 264-279.

[31] Champaloux, E. P. (2016) Dopamine Kinetics in a Drosophila Model of Parkinson Disease [dissertation], University of Virginia, Charlottesville.

[32] Greene, J. C., Whitworth, A. J., Kuo, I., Andrews, L. A., Feany, M. B., and Pallanck, L. J. (2003) Mitochondrial pathology and apoptotic muscle degeneration in Drosophila parkin mutants, Proc Natl Acad Sci U S A 100, 4078-4083.

[33] Mcgeer, P. L., Mcgeer, E. G., and Suzuki, J. S. (1977) Aging and Extrapyramidal Function, Arch Neurol-Chicago 34, 33-35.

[34] McGeer, P. L., Itagaki, S., Akiyama, H., and McGeer, E. G. (1988) Rate of cell death in parkinsonism indicates active neuropathological process, Ann Neurol 24, 574-576.

[35] Venton, B. J., and Wightman, R. M. (2003) Psychoanalytical electrochemistry: Dopamine and behavior, Analytical chemistry 75, 414a$421 a$.

[36] Xiao, N., and Venton, B. J. (2015) Characterization of dopamine releasable and reserve pools in Drosophila larvae using ATP/P2X(2)-mediated stimulation, J Neurochem 134, 445-454.

[37] Fearnley, J. M., and Lees, A. J. (1991) Aging and Parkinsons-Disease Substantia-Nigra Regional Selectivity, Brain : a journal of neurology 114, 2283-2301.

[38] Fiore, V. G., Dolan, R. J., Strausfeld, N. J., and Hirth, F. (2015) Evolutionarily conserved mechanisms for the selection and maintenance of behavioural activity, Philos TR Soc B 370. 
[39] Alekseyenko, O. V., Chan, Y. B., Li, R., and Kravitz, E. A. (2013) Single dopaminergic neurons that modulate aggression in Drosophila, $P$ Natl Acad Sci USA 110, 6151-6156.

[40] Kong, E. C., Woo, K., Li, H. Y., Lebestky, T., Mayer, N., Sniffen, M. R., Heberlein, U., Bainton, R. J., Hirsh, J., and Wolf, F. W. (2010) A Pair of Dopamine Neurons Target the D1-Like Dopamine Receptor DopR in the Central Complex to Promote Ethanol-Stimulated Locomotion in Drosophila, Plos One 5.

[41] Lebestky, T., Chang, J. S. C., Dankert, H., Zelnik, L., Kim, Y. C., Han, K. A., Wolf, F. W., Perona, P., and Anderson, D. J. (2009) Two Different Forms of Arousal in Drosophila Are Oppositely Regulated by the Dopamine D1 Receptor Ortholog DopR via Distinct Neural Circuits, Neuron 64, 522-536.

[42] Kim, Y. C., Lee, H. G., Seong, C. S., and Han, K. A. (2003) Expression of a D1 dopamine receptor dDA1/DmDOP1 in the central nervous system of Drosophila melanogaster, Gene Expr Patterns 3, 237-245.

[43] Neckameyer, W. S., Woodrome, S., Holt, B., and Mayer, A. (2000) Dopamine and senescence in Drosophila melanogaster, Neurobiol Aging 21, 145-152.

[44] White, K. E., Humphrey, D. M., and Hirth, F. (2010) The dopaminergic system in the aging brain of Drosophila, Front Neurosci 4, 205.

[45] Eusebio, A., Azulay, J. P., Ceccaldi, M., Girard, N., Mundler, O., and Guedj, E. (2012) Voxel-based analysis of whole-brain effects of age and gender on dopamine transporter SPECT imaging in healthy subjects, Eur J NuCl Med Mol I 39, 1778-1783.

[46] Sang, T. K., Chang, H. Y., Lawless, G. M., Ratnaparkhi, A., Mee, L., Ackerson, L. C., Maidment, N. T., Krantz, D. E., and Jackson, G. R. (2007) A Drosophila model of mutant human parkin-induced toxicity demonstrates selective loss of dopaminergic neurons and dependence on cellular dopamine, J Neurosci 27, 981-992.

[47] Polito, L., Greco, A., and Seripa, D. (2016) Genetic Profile, Environmental Exposure, and Their Interaction in Parkinson's Disease, Parkinsons DisUs. 
[48] Wang, C., Lu, R. F., Ouyang, X. Z., Ho, M. W. L., Chia, W., Yu, F. W., and Lim, K. L. (2007) Drosophila overexpressing parkin R275W mutant exhibits dopaminergic neuron degeneration and mitochondrial abnormalities, J Neurosci 27, 8563-8570.

[49] Pyakurel, P., Privman Champaloux, E., and Venton, B. J. (2016) Fast-Scan Cyclic Voltammetry (FSCV) Detection of Endogenous Octopamine in Drosophila melanogaster Ventral Nerve Cord, ACS Chem Neurosci.

[50] Williamson, W. R., and Hiesinger, P. R. (2010) Preparation of developing and adult Drosophila brains and retinae for live imaging, $J$ Vis Exp.

[51] Sweeney, S. T., Hidalgo, A., de Belle, J. S., and Keshishian, H. (2011) Dissection of adult Drosophila brains, Cold Spring Harb Protoc 2011, 1472-1474.

[52] Swamy, B. E. K., and Venton, B. J. (2007) Carbon nanotube-modified microelectrodes for simultaneous detection of dopamine and serotonin in vivo, Analyst 132, 876-884.

[53] Denno, M. E., Privman, E., and Venton, B. J. (2015) Analysis of Neurotransmitter Tissue Content of Drosophila melanogaster in Different Life Stages, Acs Chemical Neuroscience 6, 117-123. 
Chapter 4: Fast-scan cyclic voltammetry (FSCV) detection of endogenous octopamine in Drosophila melanogaster ventral nerve cord 


\section{Chapter 4: Fast-scan cyclic voltammetry (FSCV) detection of endogenous octopamine in Drosophila melanogaster ventral nerve cord}

\subsection{Abstract}

Octopamine is an endogenous biogenic amine neurotransmitter, neurohormone, and neuromodulator in invertebrates, and has functional analogy with norepinephrine in vertebrates. Fast-scan cyclic voltammetry (FSCV) can detect rapid changes in neurotransmitters, but FSCV has not been optimized for octopamine detection in situ. The goal of this study was to characterize octopamine release in the ventral nerve cord of Drosophila larvae for the first time. An FSCV waveform was optimized so that the potential for octopamine oxidation would not be near the switching potential where interferences can occur. Endogenous octopamine release was stimulated by genetically inserting either the ATP sensitive channel, $\mathrm{P} 2 \mathrm{X}_{2}$, or the red-light sensitive channelrhodopsin, CsChrimson, into cells expressing tyrosine decarboxylase (TDC), an octopamine synthesis enzyme. To ensure that release is due to octopamine and not the precursor tyramine, the octopamine synthesis inhibitor disulfiram was applied, and the signal decreased by $80 \%$. Stimulated release was vesicular and a $2 \mathrm{~s}$ continuous light stimulation of CsChrimson evoked 0.22 $\pm 0.03 \mu \mathrm{M}$ of octopamine release in the larval VNC. Repeated stimulations were stable with 2 or 5 minutes interstimulation times. With pulsed stimulations, the release was dependent on the frequency of applied light pulse. An octopamine transporter has not been identified, and blockers of the dopamine transporter and serotonin transporter had no significant effect on the clearance time of 
octopamine, suggesting they do not take up octopamine. This study shows that octopamine can be monitored in Drosophila, facilitating future studies of how octopamine release functions in the insect brain.

\subsection{Introduction}

Octopamine and its precursor, tyramine, are biogenic amines in invertebrates with distinct roles that range from modulation of muscles to complex social behaviors. ${ }^{1,2}$ Octopamine acts as a neurotransmitter by regulating intracellular cyclic AMP and $\mathrm{Ca}^{2+}$ in Drosophila melanogaster. ${ }^{3}$ In larvae, octopamine/tyramine neurons in the ventral nerve cord (VNC) control locomotion, and in adults, they control locomotion, sensitivity to cocaine, and egg laying activity. ${ }^{4,5}$ Octopamine also has many neuromodulatory functions including regulating neuromuscular transmission in larvae, and modulating learning, memory, and conditional courtship in adults..$^{6-8}$ When released into the hemolymph, it acts as a neurohormone to mobilize lipids and carbohydrates during energy-demanding processes. ${ }^{9,} 10$ Octopamine is found in trace amounts in vertebrates and as a trace amine may play a role in depression and other psychiatric disorders. ${ }^{11-13}$ Octopamine in invertebrates is also analogous in function to norepinephrine in vertebrates. For example, octopamine release can be stimulated by nicotine application in Drosophila, similar to norepinephrine release in mammals. ${ }^{14}$ 
Octopamine, a phenol, is an electroactive compound, and can be detected via direct electrochemistry. ${ }^{15}$ The mechanism involves a one-electron oxidation at the phenol group that creates a radical. The radical can react with other octopamine molecules to create an electroactive polymer, and a secondary oxidation peak due to oxidation of this polymer is also observed. ${ }^{16}$ Octopamine has been detected in biological samples after separation by coupling high performance liquid chromatography or capillary electrophoresis to electrochemical detection. ${ }^{17-19}$ Separation methods are useful in obtaining tissue content information, but are destructive and unable to provide information on real-time dynamics of octopamine.

The main electrochemical methods for studying real-time neurotransmitter release in vivo are amperometry, chronoamperometry, and fast-scan cyclic voltammogram (FSCV). Majdi et al. recently measured endogenous octopamine release using optogenetic stimulation in a Drosophila larval neuromuscular junction preparation. ${ }^{20}$ They used amperometry at $0.9 \mathrm{~V}$, and estimated the number of molecules of octopamine released per vesicle. Amperometry is sensitive and has high temporal resolution, but the technique is not selective and is more difficult to use in a complex preparation. Chronoamperometry measures a ratio of oxidation to reduction currents at given potentials. Fuenzalida-Uribe et al. used chronoamperometry to detect octopamine evoked by nicotine stimulation of a-bungarotoxin-sensitive nAChRs in adult Drosophila brains. ${ }^{14}$ With fast-scan cyclic voltammetry (FSCV), the voltage is ramped up and back to oxidize and 
then reduce the molecule of interest. While a background current must be subtracted, the resulting background-subtracted cyclic voltammogram (CV) helps identify the compound detected. An FSCV waveform has been optimized for stable in vitro analysis of octopamine at carbon fiber microelectrodes (CFMEs). ${ }^{16}$ Real-time measurements of dopamine and serotonin have been made in Drosophila ventral nerve cords (VNC) using FSCV but the method has not been extended to studying octopamine dynamics. ${ }^{21,22}$

Here, we optimize an FSCV waveform and detect stimulated octopamine release in the Drosophila larval ventral nerve cord (VNC) for the first time. By using a red-light activated ion channel, CsChrimson, there is no background shift close to the octopamine oxidation peak due to the photoelectric effect. ${ }^{23,24}$ Octopamine release was also verified by picospritzing octopamine into the tissue. ${ }^{25}$ CsChrimson or $\mathrm{P}_{2} \mathrm{X}_{2}$, an ATP activated channel, were expressed in neurons expressing the tyrosine decarboxylase ( $T d c 2)$, the enzyme used to synthesize the octopamine precursor, tyramine. Since these cells have both tyramine and octopamine, disulfiram, an inhibitor of tyramine $\beta$-hydroxylase (TBH), the enzyme that converts tyramine to octopamine, was used to demonstrate that the majority of the release is octopamine..$^{26-28}$ Octopamine release was vesicular and not cleared by the dopamine transporter (DAT) or serotonin transporter (SERT). These are the first measurements of endogenous octopamine release in the Drosophila larval central nervous system, which will 
facilitate studies of the function of octopamine signaling in an intact nervous system.

\subsection{Results and discussion}

\subsubsection{Visualization of octopaminergic neurons}

The UAS-GAL4 system allows targeted expression of certain proteins to specific cells or tissues. ${ }^{29}$ In this study, the GAL4 gene was expressed in cells expressing $T d c 2$, neuronal tyrosine decarboxylase (TDC). ${ }^{30}$ Tyrosine decarboxylase converts tyrosine to tyramine, which is then converted to octopamine by tyramine $\beta$-hydroxylase (TBH). The transcriptional activator, GAL4, activates UAS (Upstream Activation Sequence) and transcribes genes that are under its control - in this case genes coding for GFP, CsChrimson, or P2X $X_{2}$. Thus, those genes are expressed only in neuronal cells expressing TDC.

To characterize the location of octopaminergic projections, the UAS-GFP reporter gene was expressed under the control of Tdc2-GAL4 driver. The cell bodies, which are unpaired median neurons, are located primarily in the middle of the abdominal section of the VNC (marked a1 - a9 in Fig 4.1A) and show strong GFP expression. ${ }^{31}$ The projections extend from the middle out to the side through the neuropil. The expression pattern is similar to previously observed patterns except that here, GFP was not expressed as strongly in the thoracic section (t1 t3, Fig 4.1A). ${ }^{4}$ The electrode was therefore placed in the abdominal segment 2-4 in the neuropil, where high levels of octopaminergic projections are present. 


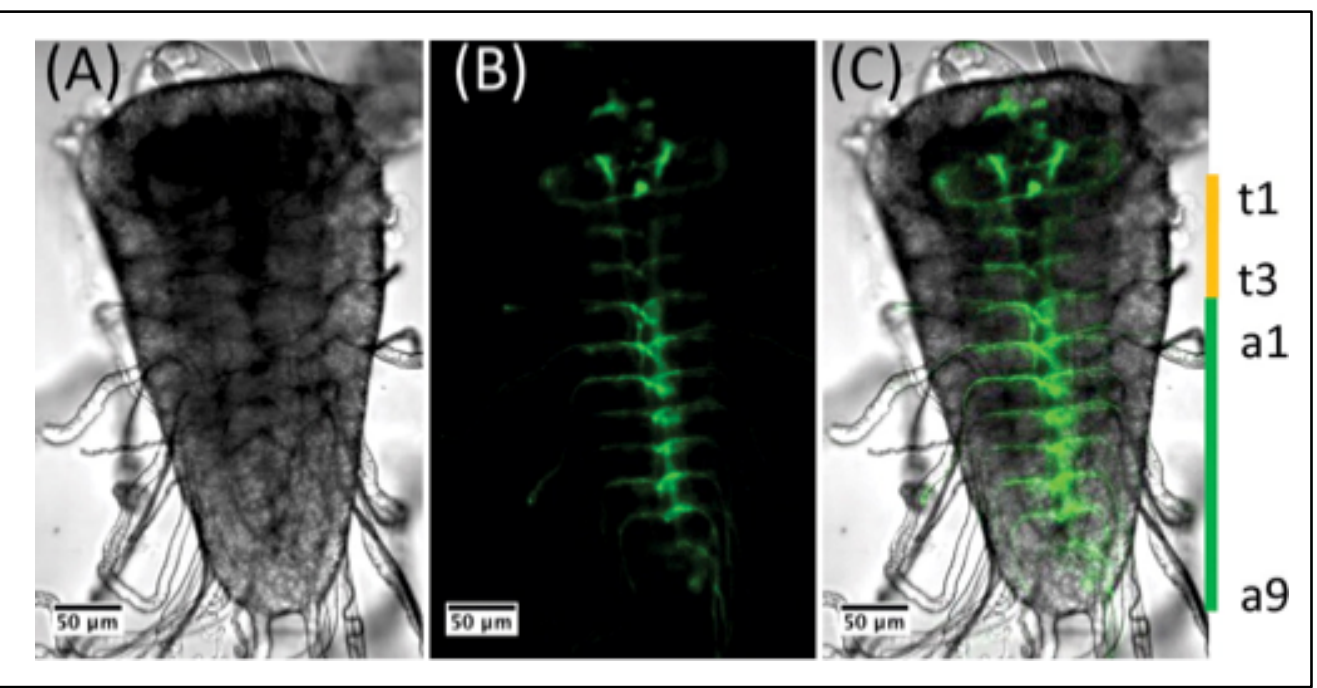

Figure 4.1. Visualization of neurons expressing tyrosine decarboxylase (Tdc) in Drosophila melanogaster larval ventral nerve cord (VNC). (A) Bright field image of a larval VNC and (B) image of the same VNC expressing GFP in neurons with tyrosine decarboxylase (Tdc2-GAL4; UAS-GFP). (C) Overlay of GFP expression on the image of the VNC. Abdominal section (a1-a9) and thoracic section ( $t 1-t 3$ ) are indicated on the right. Strong GFP expression (green) is seen on the unpaired median neurons in the abdominal section, with projections into the neuropil on either side

\subsubsection{Optimization of FSCV waveform for octopamine detection}

FSCV can be used to detect octopamine but an optimized waveform for in vivo use had not been developed. In this first study, we characterized octopamine detection with different waveforms in situ to optimize octopamine detection. First, a positive waveform, that held at a positive voltage, $0.1 \mathrm{~V}$, scanned up to $1.3 \mathrm{~V}$ and back at $600 \mathrm{~V} / \mathrm{s}$ was tested, because it had been optimized previously in vitro. ${ }^{16}$ This waveform was termed the positive waveform because of the positive holding potential. Figure 4.2A shows CsChrimson stimulated octopamine in situ (2 s stimulation) with the applied positive triangular waveform (top), cyclic voltammogram profile (middle), and color plot (bottom). The CV for the positive waveform has a peak around $1.2 \mathrm{~V}$ and the color plot shows that the oxidation peak is on the cathodic, downward scan very close to the switching potential. The 
peak potential is more shifted than for in vitro measurements, where the primary peak is observed around $0.9 \mathrm{~V}$ at this waveform, ${ }^{16}$ because the tissue can foul the electrode and slow electron transfer, changing the shape of the cyclic voltammogram. ${ }^{32}$ The positive waveform CV has very little secondary peak, because the oxidation products of octopamine are positively charged and are repelled by the positive holding potential.

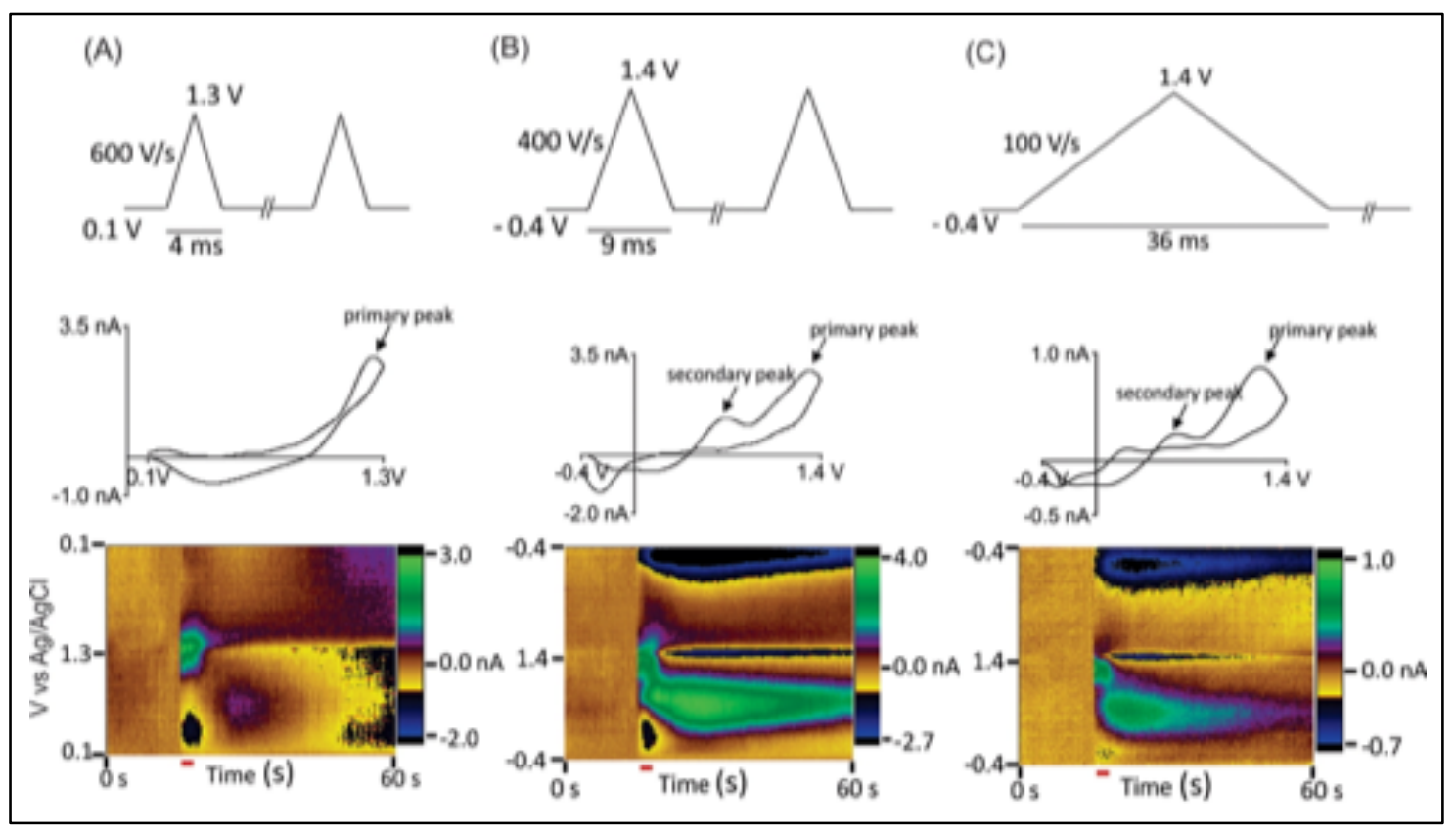

Figure 4.2. Optimization of FSCV waveform for octopamine detection. The applied waveform (top), cyclic voltammogram (center), and color plot (bottom) for octopamine detected with (A) positive waveform, (B) dopamine waveform, and (C) slower scan waveform. The $2 \mathrm{~s}$ stimulation is marked by a red line under the color plot.

Next, we tested a waveform commonly used for dopamine detection, scanning from -0.4 to $1.4 \mathrm{~V}$ and back at $400 \mathrm{~V} / \mathrm{s}$. The primary peak for octopamine is observed around $1.3 \mathrm{~V}$, close to switching potential in situ (Fig. 4.2B). The secondary peak is around $0.7 \mathrm{~V}$ and the color plot shows it lasts a long time, which suggests the oxidation products of octopamine are sticking to 
the electrode. When repeated calibration measurements are made in vitro, the response for octopamine decreases with the dopamine waveform due to fouling of the electrode by the secondary products. ${ }^{16}$

In order to move the octopamine oxidation peak away from the switching potential, we optimized a waveform using a slower scan rate than the dopamine waveform. Figure $4.2 \mathrm{C}$ shows that with a $100 \mathrm{~V} / \mathrm{s}$ scan rate, the main peak for octopamine is at $1.1 \mathrm{~V}$ on the anodic scan in situ, away from the switching potential. The secondary peak is present at $0.56 \mathrm{~V}$, which aids identification, but it is not as intense and does not last as long as for the dopamine waveform. Slower scan rates do give lower Faradaic currents, as current is proportional to scan rate. Decreasing the scan rate does decrease the sensitivity from an average of $12 \pm 1 \mathrm{nA} / \mu \mathrm{M}$ for $400 \mathrm{~V} / \mathrm{s}$ to $4.3 \pm 0.5 \mathrm{nA} / \mu \mathrm{M}$ for $100 \mathrm{~V} / \mathrm{s}$. However, noise is also proportional to background current and decreases as well. The average $\mathrm{S} / \mathrm{N}$ ratio for octopamine detection for $1 \mathrm{mM}$ octopamine with the slower scan waveform is 154 , which is $88 \%$ of the average $S / N$ ratio of 174 for the dopamine waveform. The LOD calculated from the average S/N ratios of $200 \mathrm{nM}$ octopamine for the dopamine waveform is $14 \mathrm{nM}$ and for the slower scan waveform it is $19 \mathrm{nM}^{33}$ The slower scan waveform was chosen for the rest of the studies because the background current is most unstable at the switching potential, and so errors (such as pressure changes with pressure ejection) often occur at this potential. Errors at the switching potential are a problem when using blue light sensitive Channelrhodopsin (ChR2) to mediate octopamine release 
because the photoelectric effect causes small currents at the switching potential. ${ }^{24}$ Thus, using the slower scan rate waveform keeps the main peak away from the switching potential and allows the secondary peak to be used as an identifier. While the secondary peak is present for this waveform, we show vide infra that stable current responses are observed for octopamine upon repeated stimulations.

\subsubsection{Confirmation the released compound is octopamine and that release is vesicular}

Since the CsChrimson is expressed in cells expressing the tyramine synthesis enzyme, TDC, they could be releasing octopamine or tyramine. Disulfiram, a tyramine $\beta$-hydroxylase (TBH) inhibitor, was applied to block the synthesis of octopamine from tyramine. Thus, a decrease in stimulated release after disulfiram would confirm that the release was octopamine.$^{26-28}$ Continuous, $2 \mathrm{~s}$ red light stimulations of CsChrimson were repeated every 5 min after bathing VNCs in $100 \mu \mathrm{M}$ disulfiram for $15 \mathrm{~min}$. Results were normalized to the value of the first stimulation. For control flies, release from repeated stimulations were consistent and the eighth stimulation (45 mins in buffer + DMSO) was $94 \pm 3 \%$ of the first, which was not a significant decrease (One-way ANOVA, Bonferroni post-test, $p>0.05$ ) (Fig. 4.3). Disulfiram significantly decreased evoked current (one-way ANOVA, $p<0.0001, n=6$ ), and the current decreased to $19 \pm 3 \%$ after the eighth stimulation (45 mins in disulfiram) (Bonferroni post-test, $p<0.05)$. The magnitude of the decrease is similar to the decrease in stimulated serotonin after 
the use of a serotonin synthesis inhibitor. ${ }^{34}$ This substantial decrease in current after disulfiram indicates that the majority of the evoked species is octopamine. Future studies could also confirm this genetically by blocking targeted expression of the enzyme tyramine $\beta$-hydroxylase with RNAi. However, a stable homozygous line expressing tdc2-GAL4; UAS-CsChrimson is not currently available, so all three genes - tdc2-GAL4, UAS-CsChrimson, and UAS-RNAi ${ }^{T \beta H}-$ could not be expressed together.

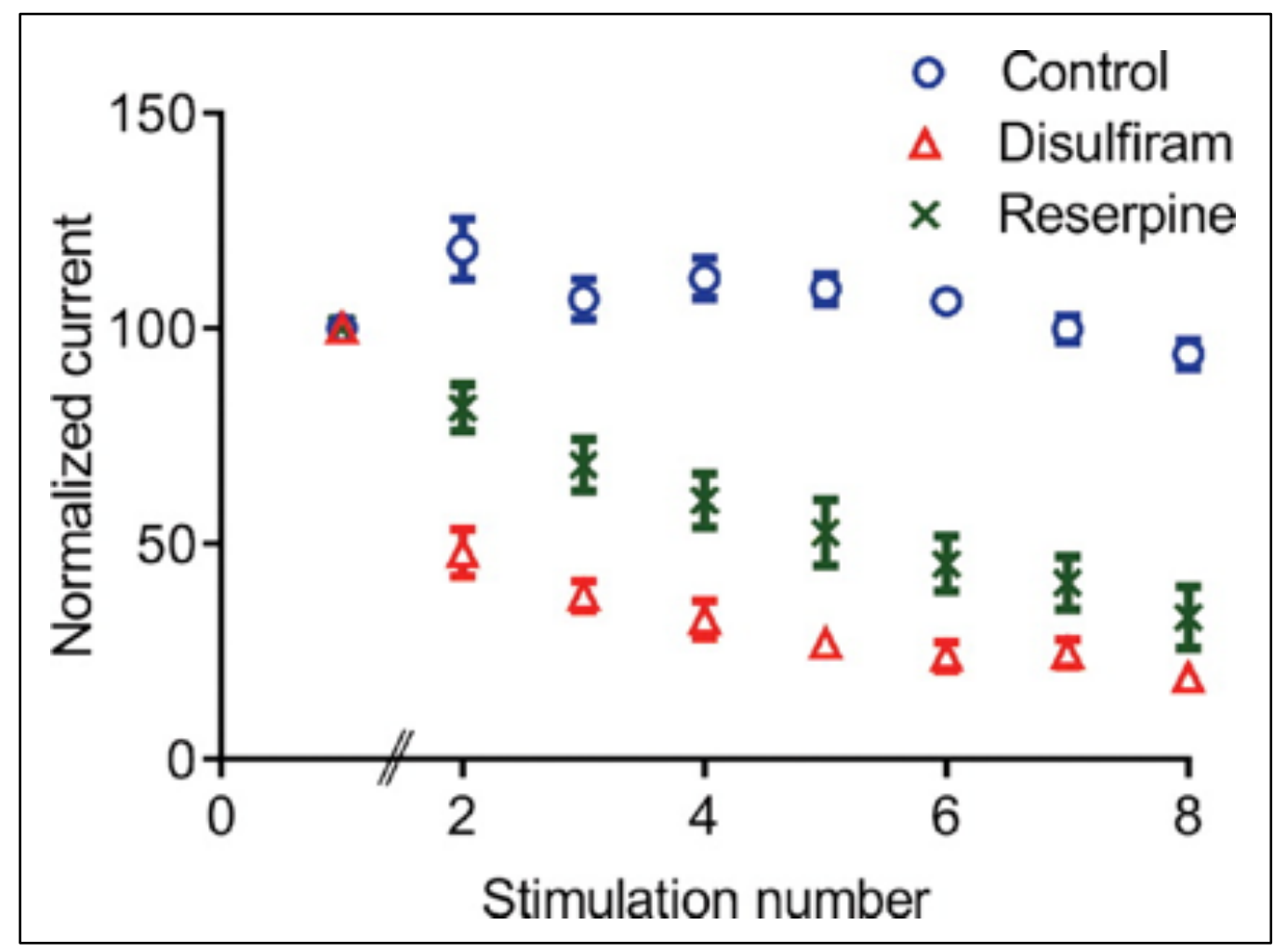

Figure 4.3. Confirmation of the detection of octopamine and that the release is vesicular. After the initial stimulation predrug (stimulation number 1), the VNCs were bathed in $100 \mu \mathrm{M}$ disulfiram to inhibit octopamine synthesis or $100 \mu \mathrm{M}$ reserpine to block the vesicular monoamine transporter. After $15 \mathrm{~min}$ in drug, stimulations were performed every $5 \mathrm{~min}$ after the second stimulation. The evoked current is significantly lower than control for both disulfiram and reserpine (one-way ANOVA, $p<0.0001, n=6$ ).

Neurotransmitters are contained in vesicles, which release their contents into the synaptic cleft by exocytosis after an action potential. To determine if 
octopamine release is vesicular, VNCs were bathed in $100 \mu \mathrm{M}$ reserpine, a vesicular monoamine transporter (VMAT) inhibitor. VMAT is a protein that transports neurotransmitters from the cytosol into synaptic vesicles for storage and subsequent release. There was a significant effect of reserpine on evoked current (one-way ANOVA, $p<0.0001, \mathrm{n}=6$ ) and evoked current decreased $33 \pm$ $7 \%$ on the eighth stimulation (45 mins in reserpine) (Bonferroni post-test, $p<$ 0.05 compared to control). Reserpine is likely not blocking $100 \%$ of VMAT or the releasable pool may not be depleted, so the response does not go to zero. The results confirm that optogenetic stimulation is causing vesicular release of octopamine. The vesicular nature of octopamine release could also be probed further with genetic manipulation of the VMAT gene in the future. ${ }^{35}$

\subsubsection{Comparison of puffed on, CsChrimson, and P2X2-mediated octopamine release}

The CVs and color plot profiles of stimulated release were compared to those obtained from puffing octopamine into VNCs. Octopamine $(0.01 \mathrm{pmol})$ was applied in the VNC using a picospritzing pipette (Fig. 4.4A). Stimulated release was compared for optically activated CsChrimson channels and ATP activated

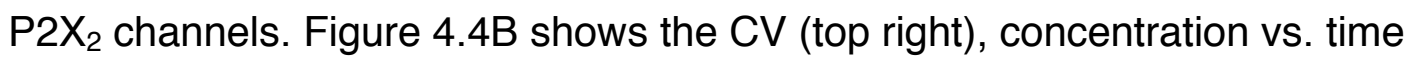
(center), and color plot (bottom) obtained for $2 \mathrm{~s}$ continuous red light stimulation of Tdc2-GAL4; UAS-CsChrimson larval VNC. On average, a $2 \mathrm{~s}$ stimulation of CsChrimson released $0.22 \pm 0.03 \mu \mathrm{M}$ octopamine. Figure $4.4 \mathrm{C}$ shows similar plots for ATP stimulation of Tdc2-GAL4; UAS-P2X larval VNC preparation. On 
average, ATP stimulation released $0.33 \pm 0.06 \mu \mathrm{M}$ of octopamine. The higher level of release with ATP compared to $2 \mathrm{~s}$ red light stimulation is consistent with previous observations of stimulated dopamine release. ${ }^{24,36}$ The CVs for octopamine stimulated by CsChrimson and $\mathrm{P} 2 \mathrm{X}_{2}$ are similar to the $\mathrm{CV}$ for exogenously applied octopamine with primary peaks around $1.1 \mathrm{~V}$ and secondary peaks around $0.5 \mathrm{~V}$. The peak close to $1.3 \mathrm{~V}$ on the $\mathrm{CV}$ for ATP stimulated release is an artifact (as marked in Figure 4.4C), which is likely due to background shifts from pressure ejection or ATP oxidation. ${ }^{36}$ With a higher scan rate waveform, this artifact would interfere with the primary oxidation peak for octopamine, but at $100 \mathrm{~V} / \mathrm{s}$, the octopamine oxidation peak and the artifact are well separated and there is no interference. The concentration vs. time plot for CsChrimson stimulated release has a sharper peak than ATP stimulated release, because the timing is easier to control with light stimulations than with ATP, which must diffuse through the tissue and is present longer. This longer course of ATP stimulation also explains the higher levels of release and longer lasting secondary peaks obtained compared to optogenetic stimulation. In future experiments, either light or ATP-mediated release could be used to study octopamine dynamics. 


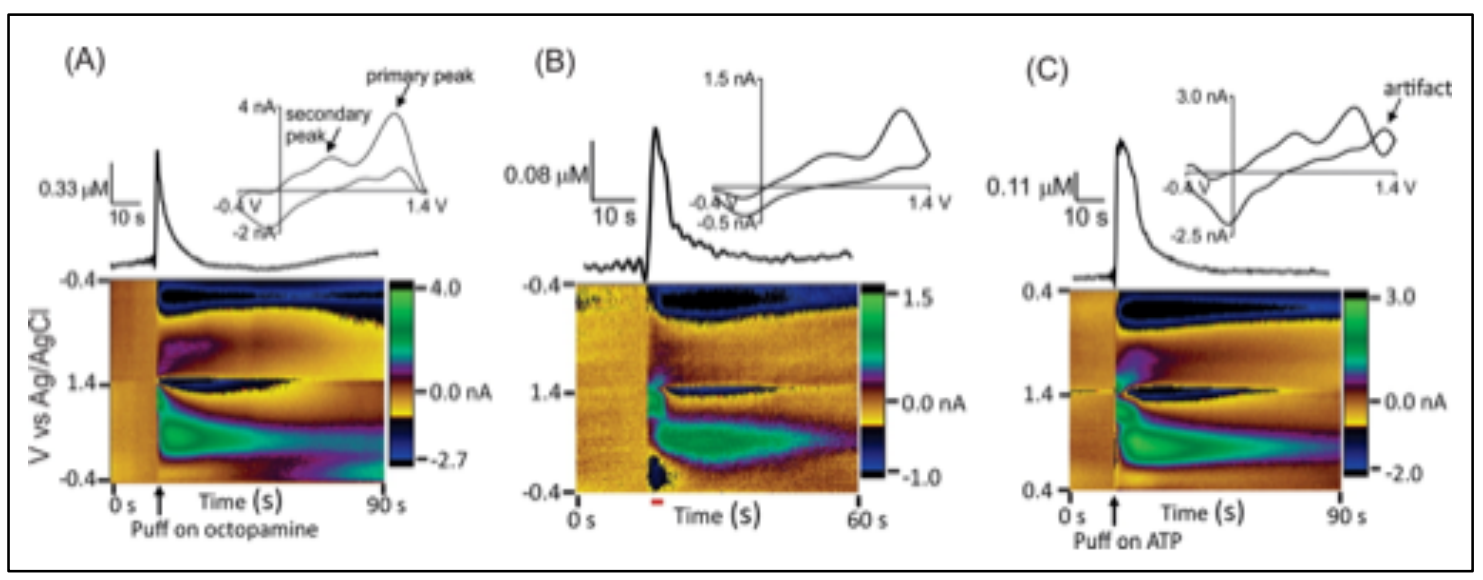

Figure 4.4. Comparison of puffed-on octopamine and different stimulations. Cyclic voltammograms (top), concentration vs time (center), and color plots (bottom) for (A) octopamine puffed into a larval VNC, (B) CsChrimson (red light, $2 \mathrm{~s}$ ) mediated release, and (C) P2X2 (0.5 pmol of ATP) mediated release in larval VNC. All show similar peak characteristics with the primary peak around $1.1 \mathrm{~V}$ and secondary peak around $0.5 \mathrm{~V}$ with the slower scan waveform.

\subsubsection{Stability of release and effect of stimulation frequency}

Red light-stimulated release via CsChrimson was further characterized by studying the stability of octopamine release and the effect of interstimulation times on stability. Two second duration, continuous red light stimulations were repeated every 2 or 5 minutes. The release was stable with both 2 minute (oneway ANOVA, $p=0.2651, \mathrm{n}=7$ ) and 5 minute (one-way ANOVA, $p=0.8197, \mathrm{n}=$ 7) interstimulation times (Fig. 4.5A). Similarly, a stable current response was also obtained upon repeated exposures to octopamine at these intervals for in vitro calibrations (one-way ANOVA, $p>0.5, n=4$ ) (Fig. 4.5B). Stable release suggests that the secondary oxidation product does not foul the electrode and cause changes in sensitivity on this time scale. The standard error of the mean was higher and currents slightly lower for the 2 minute interstimulation time; hence a 5 minute interstimulation time was used for further experiments. 


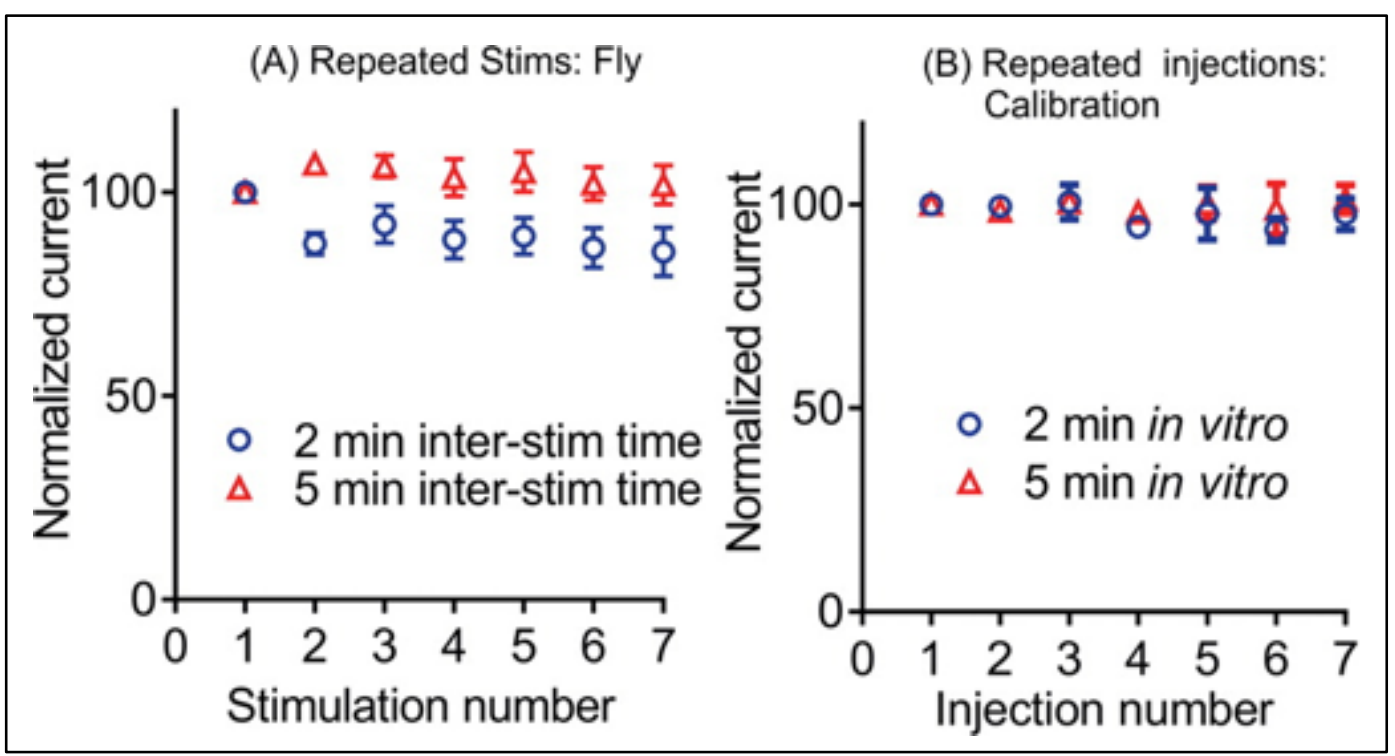

Figure 4.5. Stability of CsChrimson stimulated octopamine release. (A) The release evoked by $2 \mathrm{~s}$ red light stimulation is stable for $2 \mathrm{~min}$ interstimulation time (ANOVA, $p=0.2651, n=7$ ) and 5 min interstimulation time (ANOVA, $p=0.8197, n=7$ ). (B) Stable responses are also observed for in vitro calibrations for $2 \mathrm{~s}$ octopamine injections with $2 \mathrm{~min}($ ANOVA $p=0.7441, n=4)$ or $5 \min (p=0.9920$, $n=4$ ) interevent times.

Continuous red light stimulations were varied between $0.1 \mathrm{~s}$ and $3 \mathrm{~s}$ in order to test the effect of stimulation length on octopamine release (Fig. 4.6A). Octopamine oxidation current increased with increasing duration of light stimulation up to $0.5 \mathrm{~s}$. With stimulations longer than $1 \mathrm{~s}$, the evoked current decreased after reaching a maximum level, even while the light was still on. This decreasing response is different than the signals for dopamine and serotonin that keep increasing or reach steady state during long stimulations. ${ }^{22,34}$ The results suggest that the releasable pool for octopamine is more limited than for serotonin or dopamine in similar preparations. 


\section{(A) Continuous stim. of varying lengths}

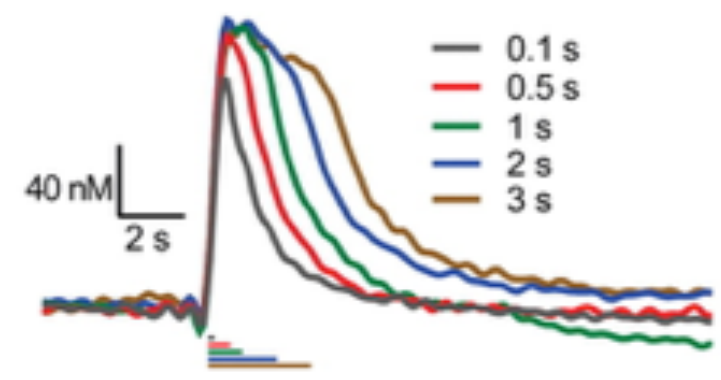

(B) Total stim. time: $2 \mathrm{~s}$

(C) Pulse number: 500
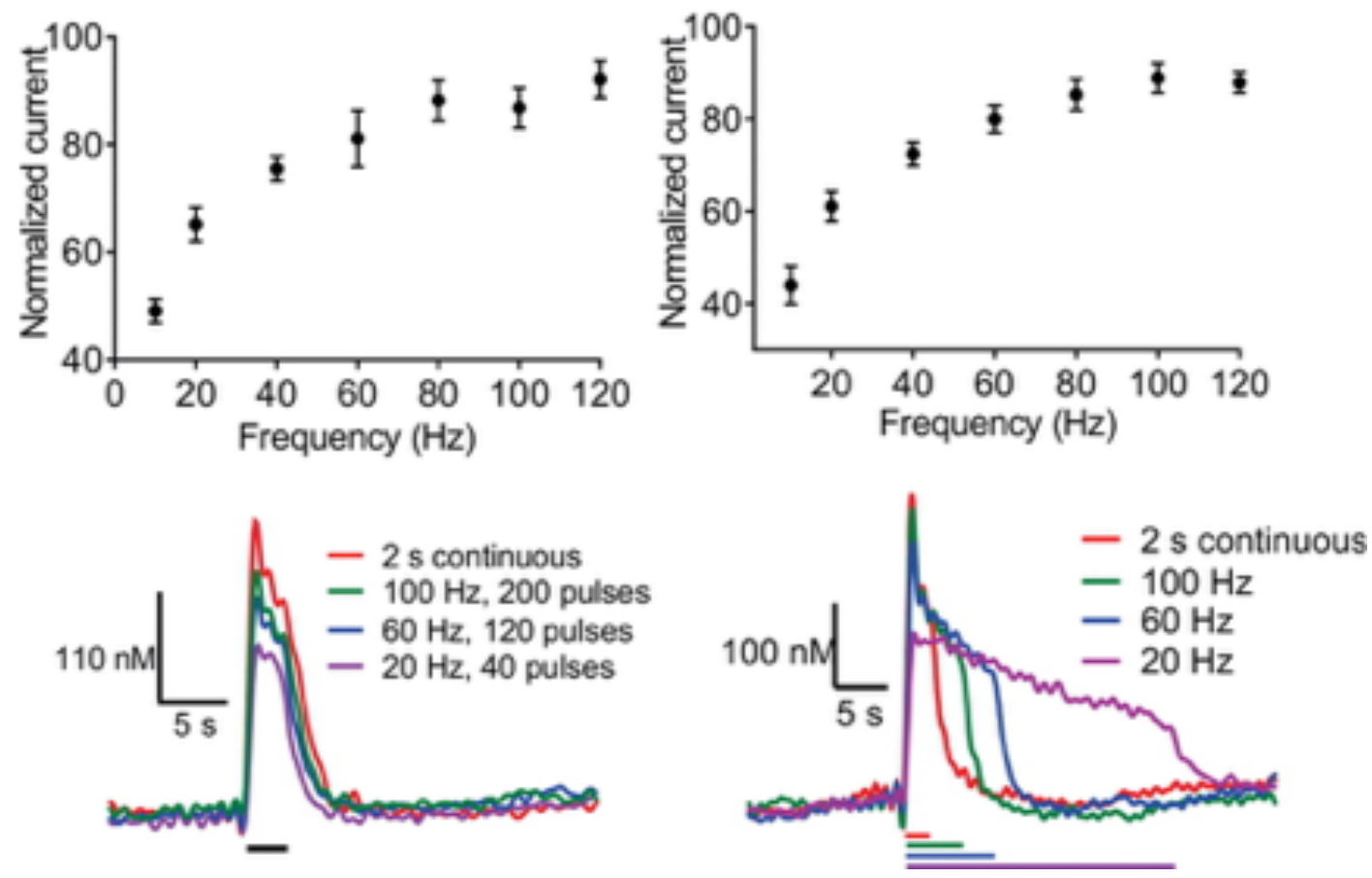

Figure 4.6. Effects of length of continuous light stimulation and frequency of pulsed stimulations. (A) Concentration vs. time plots for continuous light stimulation of different durations. Colored bars at bottom show total stimulation times. (B,C) Effect of pulsed stimulation frequency. (B) The total stimulation time was constant at $2 \mathrm{~s}$ and pulse length was $4 \mathrm{~ms}$. Data are normalized to $2 \mathrm{~s}$ continuous stimulation set to $100 \%$. Current due to evoked octopamine is dependent on the frequency of applied light and significantly different than the $2 \mathrm{~s}$ continuous stimulation up to $60 \mathrm{~Hz}$ (one-way ANOVA, Bonferonni post-test, $p<0.0001, n=7$ ). Example concentration vs. time plots are shown below with colored bars showing total stimulation time. (C) The total number of pulses was kept constant at $\mathbf{5 0 0}$ and length of pulse at $4 \mathrm{~ms}$ so the total time light is on is $2 \mathrm{~s}$. There was a significant effect of frequency up to $80 \mathrm{~Hz}$ (Bonferroni's multiple comparisons test, $p<0.05$ ). 
Pulsed light stimulations are often used to mimic neuronal firing patters. ${ }^{37,}$

${ }^{38}$ To understand the effect of pulsed stimulation and changing frequency of stimulation, pulse trains were studied in two ways: (1) holding the stimulation time constant at $2 \mathrm{~s}$ (i.e. changing the frequency and number of pulses), and (2) holding the duration of the light on constant for $2 \mathrm{~s}$ (i.e keeping the number of pulses constant at 500 pulses while changing the frequency). All light pulses were $4 \mathrm{~ms}$ in length. The currents were normalized to a $2 \mathrm{~s}$ continuous stimulation for each sample. For the first stimulation method with constant stimulation time, current was dependent on the frequency of pulsed light (Fig. 4.6B, One-way ANOVA, $p<0.0001, n=7$ ), and was significantly different from the $2 \mathrm{~s}$ continuous stimulation up to $60 \mathrm{~Hz}$ (Bonferroni's post-test, $p<0.05$ ). Figure 4.6B also shows example current vs. time traces that illustrate that higher concentrations of octopamine were released with higher frequencies. For the second stimulation method, with constant pulses, there was a significant main effect of frequency (Fig. 4.6C, one-way ANOVA, $p<0.0001, n=7$ ) and post-tests showed that signals were significantly different than the $2 \mathrm{~s}$ continuous stimulation up to $80 \mathrm{~Hz}$ (Bonferroni's post-test, $p<0.05$ ). The concentration vs. time plots (Fig. 4.6C) show that the concentration of octopamine decreases with time as the length of stimulation increases, which is clearly seen in the $20 \mathrm{~Hz}$ plot. Similar to the continuous stimulations (Fig. 4.6A), the concentration of octopamine decreases during the stimulation, which implies that the releasable pool can be easily depleted. In contrast, for other neurotransmitters like 
serotonin, a steady state response is observed at lower frequencies instead of a decrease.$^{38}$ Overall, results of the pulsed stimulation tests show the evoked current is dependent on the frequency of light; however the octopamine release is depleted with longer stimulations and decreases while the light stimulation is being applied.

\subsubsection{Characterization of octopamine clearance}

Most monoamine neurotransmitters are cleared back into cells via a transporter, but an octopamine transporter has not been identified in Drosophila. ${ }^{39}$ Octopamine has a similar structure to dopamine, and it has been suggested that the dopamine transporter could transport octopamine. ${ }^{40}$ However, tests of Drosophila dopamine transporter (dDAT) expressed in MDCK cells found that octopamine was not a good substrate for $\mathrm{dDAT} .{ }^{41}$ Drosophila serotonin transporter (dSERT) expressed in HeLa cells and Xenopus oocytes have also shown little or no activity for octopamine. ${ }^{42,43}$ However, the clearance of octopamine by known monoamine transporters has not been studied in the VNC. We tested the extent to which the dDAT or dSERT affect octopamine clearance by using nisoxetine to inhibit dDAT and fluoxetine to inhibit dSERT. ${ }^{22,44}$ Figure 4.7 shows example data before and after the uptake inhibitors. There is little effect of $20 \mathrm{mM}$ nisoxetine, and $100 \mathrm{mM}$ fluoxetine increases the peak height but not the clearance time. To quantify clearance, $t_{50}$, the time the concentration vs. time curve to decay from the peak to $50 \%$ was calculated and it was not significantly different for nisoxetine or fluoxetine compared to control values 
(paired t test, $p>0.1, \mathrm{n}=5$ - 8) (Table 1). Thus, dSERT or dDAT do not uptake octopamine.

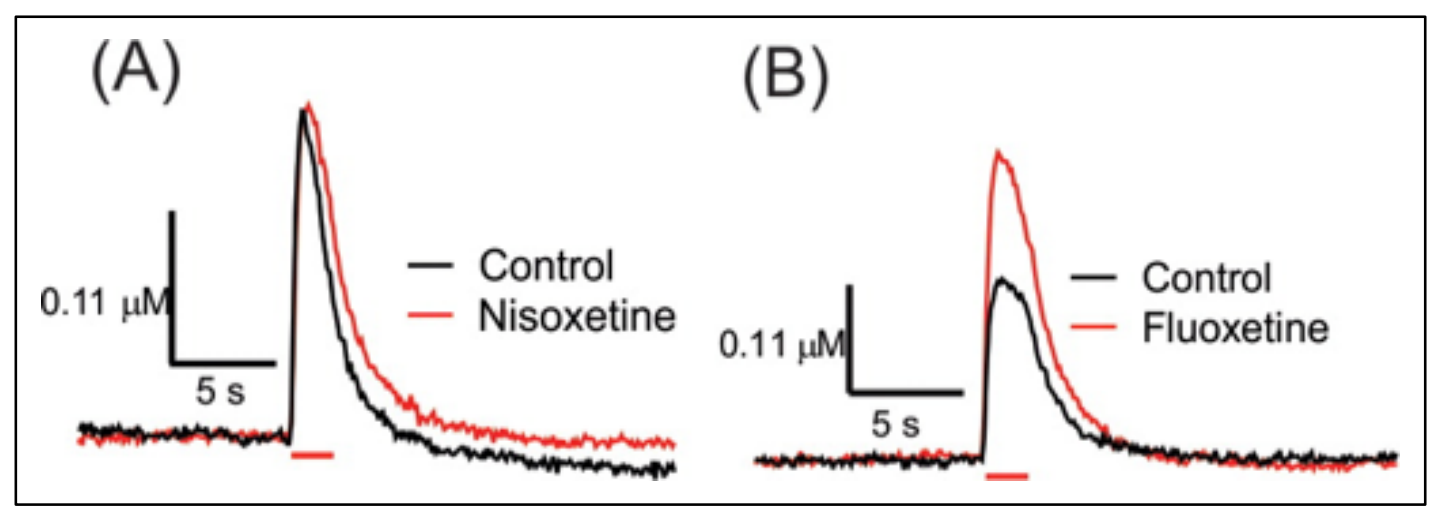

Figure 4.7. Effect of uptake inhibitors. Example concentration vs time plots for $1 \mathrm{~s}$ CsChrimson stimulation predrug (black traces) and after $15 \mathrm{~min}$ in (A) $20 \mu \mathrm{M}$ nisoxetine or (B) $100 \mu \mathrm{M}$ fluoxetine (red traces).

Table 1. Effect of uptake inhibitors on octopamine release and clearance. Effect of dopamine transporter inhibitor (nisoxetine) and serotonin transporter inhibitor (fluoxetine) on evoked concentration and clearance $\left(t_{50}\right)$ of octopamine (paired t-test). Values are reported as mean \pm SEM

\begin{tabular}{|c|l|l|l|c|}
\hline \multicolumn{1}{|c|}{ Drug } & \multicolumn{1}{|c|}{ Value } & $\begin{array}{c}\text { Before } \\
\text { Drug }\end{array}$ & After Drug & P value \\
\hline Nisoxetine, $\mathrm{n}=5$ & $\mathrm{t}_{50}(\mathrm{~s})$ & $1.4 \pm 0.1$ & $1.7 \pm 0.1$ & $p=0.1577$ \\
\hline Fluoxetine, $\mathrm{n}=7$ & $\mathrm{t}_{50}(\mathrm{~s})$ & $1.4 \pm 0.1$ & $1.4 \pm 0.1$ & $p=0.4830$ \\
\hline Nisoxetine, $\mathrm{n}=5$ & Concentration $(\mu \mathrm{M})$ & $0.16 \pm 0.02$ & $0.18 \pm 0.01$ & $p=0.4840$ \\
\hline Fluoxetine, $\mathrm{n}=7$ & Concentration $(\mu \mathrm{M})$ & $0.14 \pm 0.01$ & $0.20 \pm 0.03$ & $p=0.0078^{* *}$ \\
\hline
\end{tabular}

The $t_{50}$ value of $1.4 \mathrm{~s}$ for octopamine is lower than that for serotonin $(\sim 2.5$ s). ${ }^{38}$ The rate of diffusion will be similar for both neurotransmitters as they are in similar environment. The small $t_{50}$ value, therefore, suggests that octopamine is either metabolized quickly or transported into cells by some other transporters. N- 
acetylation is the major metabolic pathway for neurotransmitter inactivation in Drosophila, but metabolism is expected to act on a slower time scale than reuptake. ${ }^{45}$ Genome wide searches for transporter DNA have not found any other transporters for octopamine, so future research into mechanisms of clearance in Drosophila would be valuable. ${ }^{39}$

Although the $t_{50}$ values were not significantly different in the presence of the transporter inhibitors, evoked octopamine release was significantly higher in the presence of fluoxetine (paired t-test, ${ }^{* *} p=0.0078, \mathrm{n}=7$ ) (Table 1). This result is similar to previous observations of increased norepinephrine in the presence of fluoxetine in rat brains, and is another indication for the analogy between norepinephrine in vertebrates and octopamine in invertebrates. ${ }^{44}$ The increase of norepinephrine in rat brains was $5-\mathrm{HT}_{2 \mathrm{C}}$ receptor mediated, because fluoxetine is also a $5-\mathrm{HT}_{2 \mathrm{C}}$ antagonist. However, there is no $5-\mathrm{HT}_{2 \mathrm{C}}$ receptor homolog reported in Drosophila. Other 5-HT receptor homologs have been identified, such as Dm5- $\mathrm{HT}_{2 \mathrm{a}}$, but it has pharmacological properties similar to $5-\mathrm{HT}_{2 \mathrm{~B}}$ receptor in mammals and has not been identified as a $5-\mathrm{HT}_{2 \mathrm{C}}$ receptor homolog. ${ }^{46,47}$ These results suggest that future experiments might examine the ability of serotonin receptors to mediate increases in octopamine in Drosophila.

\subsubsection{Comparison of octopamine release with dopamine and serotonin} The concentration of octopamine released with $2 \mathrm{~s}$ red light stimulation $(0.22 \pm 0.03 \mu \mathrm{M})$ is slightly lower than stimulated dopamine or serotonin release in similar preparations. ${ }^{24,34}$ For both serotonin and dopamine release, a $2 \mathrm{~s}$ 
continuous light stimulation produces a peak that after the stimulation ends, but for octopamine, the peak release begins to decrease during the stimulation for a $1 \mathrm{~s}$ continuous stimulation. These results suggest a smaller octopamine releasable pool, which was unexpected as octopamine tissue content is higher than both serotonin or dopamine in the larval VNCs. ${ }^{17}$ There are several reasons why the stimulated octopamine release could be lower than expected. First, the lower release could mean that $T d c 2-G A L 4$ is not as strong a driver as that used for dopaminergic (TH-GAL4) and serotoninergic (TPH-GAL4) neurons. The Tdc2GAL4 line used here (Bloomington fly stock 9313) was not made utilizing the sitespecificity of attB and attP on DNA and could have resulted in less expression of the driver and thus CsChrimson expression would be lower. ${ }^{48}$ Second, octopamine transporters have not been identified in Drosophila and the lower release could be because there is no rapid recycling of octopamine through transporters to continually replenish the releasable pool. ${ }^{39}$ Finally, the release of octopamine was measured in the neuropil of the VNC, but the GFP images (Fig. 4.1) indicate that there may not be as much arborization and terminal as there are for dopaminergic or serotonergic terminals in that region. Future studies could examine regional differences in octopamine release both in the larval and also adult CNS of Drosophila.

\subsection{Conclusions}

We have detected and quantified stimulated endogenous octopamine release with FSCV for the first time. Optogenetic stimulation or activation of an exogenous ATP channel was used to stimulate vesicular octopamine release. 
Release was stable with 2 or 5 minutes interstimulation times, and dependent on the frequency of the applied light. Long stimulations cause decreasing response, so stimulations of $1 \mathrm{~s}$ or shorter should be used in future studies. Although the clearance of octopamine was rapid, there was no evidence of octopamine transport through dopamine or serotonin transporters suggesting an alternative method of clearance. The serotonin transporter inhibitor, fluoxetine, did increase the amount of octopamine release, suggesting that future studies are needed to investigate the effect of serotonin on octopamine release. Overall, FSCV can be used to measure octopamine release and clearance rates in larvae, providing insight into the mechanisms of action of this invertebrate neurotransmitter.

\subsection{Experimental Section}

\subsubsection{Chemicals}

All chemicals were purchased from Sigma-Aldrich (St. Louis, MO), and solutions were prepared in Milli Q water (Millipore, Billerica, MA) unless noted otherwise. Electrode calibrations were performed in phosphate buffer solution (PBS) (131.25 mM NaCl, $3.0 \mathrm{mM} \mathrm{KCl,} 10.0 \mathrm{mM} \mathrm{NaH}_{2} \mathrm{PO}_{4}, 1.2 \mathrm{mM} \mathrm{MgCl}_{2}, 2.0$ $\mathrm{mM} \mathrm{Na}_{2} \mathrm{SO}_{4}$, and $1.2 \mathrm{mM} \mathrm{CaCl}_{2}$ ) with $\mathrm{pH}$ adjusted to 7.4 , which was made once a month and stored at $4^{\circ} \mathrm{C}$. To make the larval dissection buffer, $11.1 \mathrm{mM}$ glucose and $5.3 \mathrm{mM}$ trehalose were added to the PBS buffer on the day of the experiment. For $\mathrm{P}_{2} \mathrm{X}_{2}$ experiments, ATP solution $(0.1 \mathrm{mM})$ was also prepared daily in the dissection buffer. Ten $\mathrm{mM}$ stock solutions of octopamine were prepared in $0.1 \mathrm{M} \mathrm{HClO}_{4}$ once a month and stored at $4^{\circ} \mathrm{C}$. Diluted octopamine 
solutions for electrode calibration and pressure ejection experiments were prepared daily in PBS buffer.

Disulfiram (Tocris Bioscience, Ellisville, MO) and reserpine (Sigma-Aldrich, St. Louis, MO) stock solutions of $10 \mathrm{mM}$ were prepared in DMSO, and diluted to $400 \mu \mathrm{M}$ in PBS. Fluoxetine $(400 \mu \mathrm{M})$ and nisoxetine $(80 \mu \mathrm{M})$ were prepared in PBS. To add drug to the VNC, $1 \mathrm{~mL}$ of the respective solution was added to the Petri dish that contained $3 \mathrm{~mL}$ of dissection buffer.

\subsubsection{Drosophila and VNC preparation}

Drosophila melanogaster stocks were maintained and crossed as described before. ${ }^{24}$ Males with Tdc2-GAL4 on the second chromosome (Bloomington fly stock \#9313, gift of Jay Hirsh, University of Virginia) were used to express the following UAS constructs (on the third chromosome): UASCsChrimson and UAS-P2X 2 (gifts of Vivek Jayaraman, Janelia Farm Research Campus) by crossing Tdc2-GAL4 males with respective virgin females. Resulting tdc2-GAL4; UAS-CsChrimson larvae were fed standard cornmeal food with 400 $\mu \mathrm{M}$ all-trans-retinal and kept in the dark. Larvae were dissected in phosphate buffer and prepared using previously described procedures. ${ }^{24}$ Briefly, the central nervous system was dissected out from a third instar larva using fine tweezers, the optic lobes cut off, and the VNC was transferred to the lid of a Petri dish with $3 \mathrm{~mL}$ of the dissection buffer in it. For ATP stimulation and octopamine pressure ejection studies, the opposite end of the VNC was cut with fine scissors to facilitate micropipette insertion as described previously. ${ }^{49}$ 


\subsubsection{Electrochemical setup, light stimulation, and data analysis}

Carbon-fiber microelectrodes (CFMEs) were fabricated with $7 \mu \mathrm{m}$ diameter T-650 carbon fibers (Cytec Engineering Materials, West Patterson, NJ) in a $1.2 \mathrm{~mm}$ o.d. glass capillary (A-M systems, Carlsburg, WA) pulled to a tip. CFMEs were cut to 50 - $75 \mu \mathrm{m}$ to form cylindrical electrodes. Data were collected with Dagan Chem-Clamp potentiostat (Dagan, Minneapolis, MN, $n=0.01$ headstage), PCl 6711 and 6052 computer interface cards (National Instruments, Austin, TX) and a home-built breakout box. Tar Heel CV software (gift of Mark Wightman, University of North Carolina) was used for data collection and analysis. Electrodes were precalibrated with $1 \mu \mathrm{M}$ octopamine in a flow cell. Electrodes were also calibrated in the presence of disulfiram and reserpine in the buffer to test any effects on sensitivity. There was no significant effect of either disulfiram or reserpine on electrode sensitivity (paired t-test, $p>0.1, \mathrm{n}=4$ )

The VNC and electrode were viewed under a 40X water immersion lens (Carl Zeiss Microscopy, LLC, Thornwood, NY, USA) and the electrode was inserted in the neuropil from the second to forth abdominal section using a micromanipulator (Narshige N-MMN-1 and N-MMO-202ND). The potential was applied between the CFME and a $\mathrm{Ag} / \mathrm{AgCl}$ reference electrode in the bath. Optical stimulation was performed with $617 \mathrm{~nm}$ LED $(\sim 0.2 \mathrm{~mW})$ and driver (ThorLabs, Newton, NJ) with optical fiber placed $\sim 400 \mu \mathrm{m}$ from the VNC.

A picospritzing pipette was made with the same glass capillary and vertical puller used to fabricate CFMEs. After being pulled, the pipette tips were 
trimmed and polished to a $30^{\circ}$ angle using a microelectrode beveller (Sutter Instrument Co., Novato, CA, USA). The pipette was filled with $0.1 \mathrm{mM}$ ATP and inserted into the VNC to pressure eject ATP using Picospritzer III instrument (Parker Hannifin, Fairfield, NJ). The pipette was calibrated by measuring the diameter of liquid ejected in oil at a set pressure and ejection time. A similar procedure was used for pressure ejection of $20 \mu \mathrm{M}$ octopamine.

All statistics were performed with GraphPad Prism 6 (GraphPad Software, Inc., La Jolla, CA). Significance was measured with a 95\% confidence interval. Graphs are shown as mean \pm standard error of the mean. 


\subsection{References}

[1] Roeder, T. (1999) Octopamine in invertebrates, Progress in neurobiology 59, 533-561.

[2] Farooqui, T. (2012) Review of octopamine in insect nervous system, Open access insect physiology 4, 1-17.

[3] Balfanz, S., Strunker, T., Frings, S., and Baumann, A. (2005) A family of octopamine [corrected] receptors that specifically induce cyclic AMP production or $\mathrm{Ca} 2+$ release in Drosophila melanogaster, Journal of neurochemistry 93, 440-451.

[4] Selcho, M., Pauls, D., El Jundi, B., Stocker, R. F., and Thum, A. S. (2012) The role of octopamine and tyramine in Drosophila larval locomotion, J Comp Neurol 520, 3764-3785.

[5] Hardie, S. L., Zhang, J. X., and Hirsh, J. (2007) Trace amines differentially regulate adult locomotor activity, cocaine sensitivity, and female fertility in Drosophila melanogaster, Dev Neurobiol 67, 1396-1405.

[6] Nishikawa, K., and Kidokoro, Y. (1999) Octopamine inhibits synaptic transmission at the larval neuromuscular junction in Drosophila melanogaster, Brain Res 837, 67-74.

[7] Schwaerzel, M., Monastirioti, M., Scholz, H., Friggi-Grelin, F., Birman, S., and Heisenberg, M. (2003) Dopamine and octopamine differentiate between aversive and appetitive olfactory memories in Drosophila, $J$ Neurosci 23, 10495-10502.

[8] Zhou, C., Huang, H., Kim, S. M., Lin, H. W., Meng, X. L., Han, K. A., Chiang, A. S., Wang, J. W., Jiao, R. J., and Rao, Y. (2012) Molecular Genetic Analysis of Sexual Rejection: Roles of Octopamine and Its Receptor OAMB in Drosophila Courtship Conditioning, J Neurosci 32, 14281-14287.

[9] Orchard, I., Ramirez, J. M., and Lange, A. B. (1993) A Multifunctional Role for Octopamine in Locust Flight, Annu Rev Entomol 38, 227-249.

[10] Fields, P. E., and Woodring, J. P. (1991) Octopamine Mobilization of Lipids and Carbohydrates in the House Cricket, Acheta-Domesticus, $J$ Insect Physiol 37, 193-199. 
[11] Sandler, M., Ruthven, C. R. J., Goodwin, B. L., Reynolds, G. P., Rao, V. A. R., and Coppen, A. (1979) Deficient Production of Tyramine and Octopamine in Cases of Depression, Nature 278, 357-358.

[12] Axelrod, J., and Saavedra, J. M. (1977) Octopamine, Nature 265, 501-504.

[13] D'andrea, G., Nordera, G., Pizzolato, G., Bolner, A., Colavito, D., Flaibani, R., and Leon, A. (2010) Trace amine metabolism in Parkinson's disease: Low circulating levels of octopamine in early disease stages, Neurosci Lett 469, 348-351.

[14] Fuenzalida-Uribe, N., Meza, R. C., Hoffmann, H. A., Varas, R., and Campusano, J. M. (2013) nAChR-induced octopamine release mediates the effect of nicotine on a startle response in Drosophila melanogaster, Journal of neurochemistry 125, 281-290.

[15] Bailey, B. A., Martin, R. J., and Downer, R. G. H. (1982) Simultaneous Determination of Dopamine, Norepinephrine, Tyramine and Octopamine by Reverse-Phase High-Performance Liquid-Chromatography with Electrochemical Detection, J Liq Chromatogr 5, 2435-2452.

[16] Cooper, S. E., and Venton, B. J. (2009) Fast-scan cyclic voltammetry for the detection of tyramine and octopamine, Analytical and bioanalytical chemistry 394, 329-336.

[17] Denno, M. E., Privman, E., and Venton, B. J. (2015) Analysis of neurotransmitter tissue content of Drosophila melanogaster in different life stages, ACS Chem Neurosci 6, 117-123.

[18] Fang, H. F., Vickrey, T. L., and Venton, B. J. (2011) Analysis of Biogenic Amines in a Single Drosophila Larva Brain by Capillary Electrophoresis with Fast-Scan Cyclic Voltammetry Detection, Analytical chemistry 83, 2258-2264.

[19] Fuzeau-Braesch, S. P., C. (1983) Determination of octopamine using high pressure liquid chromatography and electrochemical detection: studies of the brain of the cricket Locusta migratoria cinerascens, Agressologie: revue internationale de physio-biologie et de pharmacologie appliquees aux effets de l'agression 24, 377-379.

[20] Majdi, S., Berglund, E. C., Dunevall, J., Oleinick, A. I., Amatore, C., Krantz, D. E., and Ewing, A. G. (2015) Electrochemical Measurements of 
Optogenetically Stimulated Quantal Amine Release from Single Nerve Cell Varicosities in Drosophila Larvae, Angewandte Chemie.

[21] Borue, X., Condron, B., and Venton, B. J. (2010) Both synthesis and reuptake are critical for replenishing the releasable serotonin pool in Drosophila, Journal of neurochemistry 113, 188-199.

[22] Vickrey, T. L., Condron, B., and Venton, B. J. (2009) Detection of endogenous dopamine changes in Drosophila melanogaster using fastscan cyclic voltammetry, Analytical chemistry 81, 9306-9313.

[23] Klapoetke, N. C., Murata, Y., Kim, S. S., Pulver, S. R., Birdsey-Benson, A., Cho, Y. K., Morimoto, T. K., Chuong, A. S., Carpenter, E. J., Tian, Z., Wang, J., Xie, Y., Yan, Z., Zhang, Y., Chow, B. Y., Surek, B., Melkonian, M., Jayaraman, V., Constantine-Paton, M., Wong, G. K., and Boyden, E. S. (2014) Independent optical excitation of distinct neural populations, Nature methods 11, 338-346.

[24] Privman, E., and Venton, B. J. (2015) Comparison of Dopamine Kinetics in the Larval Drosophila Ventral Nerve Cord and Protocerebrum with Improved Optogenetic Stimulation, Journal of neurochemistry.

[25] Ding, S., and Sachs, F. (1999) Single channel properties of P2X2 purinoceptors, J Gen Physiol 113, 695-720.

[26] Musacchio, J. K., Irwia J.; Snyder, Solomon. (1964) Effects of disulfiram on tissue norepinephrine content and subcellular distribution of dopamine, tyramine and their b-hydroxylated metabolites, Life Sciences 3, 769-775.

[27] Carlsson, A., Henning, M., Lindberg, P., Martinson, P., Trolin, G., Waldeck, B., and Wickberg, B. (1978) On the disulfiram-like effect of coprine, the pharmacologically active principle of Coprinus atramentarius, Acta pharmacologica et toxicologica 42, 292-297.

[28] Lee, F. L., Weiner, N., and Trendelenburg, U. (1967) The uptake of tyramine and formation of octopamine in normal and tachyphylactic rat atria, $J$ Pharmacol Exp Ther 155, 211-222.

[29] Brand, A. H., and Perrimon, N. (1993) Targeted Gene-Expression as a Means of Altering Cell Fates and Generating Dominant Phenotypes, Development 118, 401-415. 
[30] Cole, S. H., Carney, G. E., McClung, C. A., Willard, S. S., Taylor, B. J., and Hirsh, J. (2005) Two functional but noncomplementing Drosophila tyrosine decarboxylase genes: distinct roles for neural tyramine and octopamine in female fertility, J Biol Chem 280, 14948-14955.

[31] Vomel, M., and Wegener, C. (2008) Neuroarchitecture of aminergic systems in the larval ventral ganglion of Drosophila melanogaster, Plos One 3, e1848.

[32] Amatore, C., Saveant, J. M., and Tessier, D. (1983) Charge-Transfer at Partially Blocked Surfaces - a Model for the Case of Microscopic Active and Inactive Sites, J Electroanal Chem 147, 39-51.

[33] Morgan, D. M., and Weber, S. G. (1984) Noise and signal-to-noise ratio in electrochemical detectors, Analytical chemistry 56, 2560-2567.

[34] Borue, X., Cooper, S., Hirsh, J., Condron, B., and Venton, B. J. (2009) Quantitative evaluation of serotonin release and clearance in Drosophila, $J$ Neurosci Methods 179, 300-308.

[35] Koob, G. F., and Simon, E. J. (2009) The Neurobiology of Addiction: Where We Have Been and Where We Are Going, J Drug Issues 39, 115-132.

[36] Xiao, N., and Venton, B. J. (2015) Characterization of dopamine releasable and reserve pools in Drosophila larvae using ATP/P2X(2)-mediated stimulation, J Neurochem 134, 445-454.

[37] Bass, C. E., Grinevich, V. P., Vance, Z. B., Sullivan, R. P., Bonin, K. D., and Budygin, E. A. (2010) Optogenetic control of striatal dopamine release in rats, Journal of neurochemistry 114, 1344-1352.

[38] Xiao, N., Privman, E., and Venton, B. J. (2014) Optogenetic control of serotonin and dopamine release in Drosophila larvae, ACS Chem Neurosci 5, 666-673.

[39] Donly, B. C., and Caveney, S. (2005) A transporter for phenolamine uptake in the arthropod CNS, Archives of insect biochemistry and physiology 59, 172-183.

[40] Martin, C. A., and Krantz, D. E. (2014) Drosophila melanogaster as a genetic model system to study neurotransmitter transporters, Neurochemistry International 73, 71-88. 
[41] Porzgen, P., Park, S. K., Hirsh, J., Sonders, M. S., and Amara, S. G. (2001) The antidepressant-sensitive dopamine transporter in Drosophila melanogaster: A primordial carrier for catecholamines, Mol Pharmacol 59, 83-95.

[42] Corey, J. L., Quick, M. W., Davidson, N., Lester, H. A., and Guastella, J. (1994) A Cocaine-Sensitive Drosophila Serotonin Transporter - Cloning, Expression, and Electrophysiological Characterization, P Natl Acad Sci USA 91, 1188-1192.

[43] Demchyshyn, L. L., Pristupa, Z. B., Sugamori, K. S., Barker, E. L., Blakely, R. D., Wolfgang, W. J., Forte, M. A., and Niznik, H. B. (1994) Cloning, Expression, and Localization of a Chloride-Facilitated, Cocaine-Sensitive Serotonin Transporter from Drosophila-Melanogaster, P Natl Acad Sci USA 91, 5158-5162.

[44] Bymaster FP, Z. W., Carter PA, Shaw J, Chernet E, Phebus L, Wong DT, Perry KW. (2002) Fluoxetine, but not other selective serotonin uptake inhibitors, increases norepinephrine and dopamine extracellular levels in prefrontal cortex., Psychopharmacology (Berl) 160, 353-361.

[45] Paxon, T. L., Powell, P. R., Lee, H. G., Han, K. A., and Ewing, A. G. (2005) Microcolumn separation of amine metabolites in the fruit fly, Analytical chemistry $77,5349-5355$.

[46] Colas, J. F., Launay, J. M., Kellermann, O., Rosay, P., and Maroteaux, L. (1995) Drosophila 5-HT2 serotonin receptor: coexpression with fushitarazu during segmentation, Proc Natl Acad Sci U S A 92, 5441-5445.

[47] Clark, M. C., Dever, T. E., Dever, J. J., Xu, P., Rehder, V., Sosa, M. A., and Baro, D. J. (2004) Arthropod 5-HT2 receptors: a neurohormonal receptor in decapod crustaceans that displays agonist independent activity resulting from an evolutionary alteration to the DRY motif, $J$ Neurosci 24 , 3421-3435.

[48] Hales, K. G., Korey, C. A., Larracuente, A. M., and Roberts, D. M. (2015) Genetics on the Fly: A Primer on the Drosophila Model System, Genetics 201, 815-842.

[49] Vickrey, T. L., Xiao, N., and Venton, B. J. (2013) Kinetics of the Dopamine Transporter in Drosophila Larva, Acs Chemical Neuroscience 4, 832-837. 
Chapter 5: Conclusions and Future Directions 


\section{Chapter 5: Conclusions and Future Directions}

This thesis describes different methods to stimulate neurotransmitter release in Drosophila melanogaster CNS and ways to optimize the FSCV waveform for electrochemical detection of a neurotransmitter. In the second chapter, I described a new method of using acetylcholine and other nAChR agonists to stimulate dopamine release in Drosophila melanogaster larval ventral nerve cord (VNC). Acetylcholine stimulates endogenous channels and enables studies of Drosophila that don't express any transgenic channels. Mutations in nAChR subunits affected neonicotinoid-stimulated dopamine release without altering the response to nicotine. Therefore our method allows studies of how mutations affect the neurochemical properties of nAChRs, which are important targets for insecticides. Using the new stimulation method, evoked dopamine was also measured in adult Drosophila brains, as described in Chapter 3. I studied stimulated dopamine release in control and Parkinson's disease model adult Drosophila at different ages. This was the first time we detected evoked dopamine in adults. Our lab has tried to study optogenetically-stimulated dopamine release in transgenic adults with little success. My work, therefore, makes it possible to study dopamine neurotransmission in adult Drosophila melanogaster. The fourth chapter describes optimization of a FSCV waveform for octopamine detection in situ, and characterization of stimulated octopamine in Drosophila larval VNC. Octopamine is a major insect neurotransmitter that is analogous to norepinephrine in mammals. It oxidizes at a higher potential than dopamine and the oxidation peak appears close to the switching potential with 
the traditional dopamine waveform. Also, octopamine is a phenol and its oxidation products form polymers that foul the electrode surface. Detection of octopamine in Drosophila has been hampered due to these limitations. With the FSCV waveform I have optimized, we can now detect and quantitate evoked octopamine. This enables the study of octopamine neurotransmission and how it changes in disease models or in mutant lines.

In this final chapter, I summarize the main conclusions and suggest future directions for the field. The work described in this thesis allows the study of stimulated dopamine release in most Drosophila lines. Previous research required the expression of transgenic channels to study dopamine release, but that is no longer a limitation. The method has also allowed us to move into adults and study changes of dopamine neurochemistry with aging and in flies that model age-related human disease. Finally, the method developed for the detection of octopamine in situ will enable future studies of the neurochemistry of this important invertebrate neurotransmitter.

\section{1. $\mathrm{nAChR}$ mediated dopamine release in Drosophila melanogaster}

In the second chapter, I have described how nAChR agonists stimulate dopamine release in Drosophila larvae. In previous studies, neurotransmitter release was mediated by exogenous channels expressed in either dopaminergic or serotonergic cells. ${ }^{1-3}$ The channels were activated by light (ChR2 or CsChrimson) or ATP (P2X 2 . While expression of transgenes is relatively simple in Drosophila, it can be a disadvantage when lines that don't express the 
channels need to be tested or when many genes must be expressed simultaneously. For example, the Parkin-RNAi flies have a mutation for Parkin on their third chromosome and to insert the exogenous channel, it must be expressed using a different chromosome and balancers. Therefore, we developed a method to evoke endogenous neurotransmitter release in flies that don't express special channels.

Since acetylcholine stimulation evokes endogenous dopamine by activating endogenous nAChR channels, the method can be used to study release in wild type flies or disease mutants that do no express exogenous ion channels. We have previously not been able to detect optogenetically-stimulated dopamine in adult Drosophila flies (due to low expression of the channel), but can now study it with acetylcholine stimulation as described in Chapter 3 . This method is very useful for initial screening of mutations that could affect dopamine; we can take any fly and easily measure the acetylcholine-stimulated release at any age without having to make any mutations. For example, we can study effects of various disease mutations, such as mutations of different genes associated with Parkinson's disease and how they affect dopamine release, clearance and dynamics in the brain. Thus, we expect many preliminary studies to see the effects of genes on neurochemistry that will be performed with this method in the future.

We used acetylcholine, nicotine, or neonicotinoids to stimulate dopamine release in wild type Drosophila larval VNC. We discovered that nicotine and 
neonicotinoids evoke release that last longer than release evoked by acetylcholine. This provides evidence for the mechanism of how nicotine and neonicotinoids act as insecticides by overstimulating nAChRs. Our method allows studies of the effects of neonicotinoids on the neurochemistry of insects and can be extended to mammals to understand the extent to which neonicotinoids affect mammalian nAChRs. This can also aid in the discovery of neonicotinoids that affect insects but only minimally affect mammals. The possibility to study neurochemical effects of $\mathrm{nAChR}$ and its subunit mutations on neonicotinoid evoked responses also opens doors to use the Drosophila model organism for rapid screening of $n A C h R$ subunit mutations that contribute to insecticide resistance.

nAChRs are important insecticide targets, and insect nAChRs are difficult to express in host cells. ${ }^{4}$ In flies that have mutations in the nAChR subunits and are resistant to the neonicotinoids, there was no neonicotinoid-stimulated release, which suggests that mechanisms for stimulated dopamine release and resistance development are linked. This validates the use of our method to study the neurochemical effects of nAChR mutations, and future use of Drosophila to understand insecticide resistance better. Drosophila melanogaster is a widely used model organism to study the nervous system and there are several toolkits available for its genetic manipulation. Our method enables investigations of how small changes in the nAChRs affect their sensitivity to various agonists including neonicotinoids. Future studies can investigate how dopamine release and 
clearance are affected by changes in the nAChR subunits, which will lead to better understanding of target site specificity of agonists. Neurochemical measurements in Drosophila can therefore be used to understand neonicotinoid resistance development to develop better resistance management methods.

Another area of investigation that my research enables is the study of effects of nicotine on nAChRs. Nicotine causes dopamine release in the brains in mammals, and I show here that there is dopamine response in Drosophila as well. How different $\mathrm{nAChR}$ subunits play a role in addiction and withdrawal symptoms in humans is not well understood. Since dopamine in involved in the reward pathway, the method I described here can be used to understand the effects of different subunit mutations in regulating dopamine dynamics. Future research can investigate how changes in the different $\alpha$ and $\beta$ subunit affect dopamine response in Drosophila, and provide an understanding of how addiction and withdrawal symptoms can be managed better.

\subsection{Evoked dopamine release in adult Drosophila and in Parkinson's disease model}

As discussed earlier, we have extensively studied stimulated neurotransmitter release in Drosophila larval VNC in our lab. ${ }^{1-3}$ For the first time, we studied evoked dopamine release in adult Drosophila brains with acetylcholine stimulation. The central complex region of the adult brain is rich in dopaminergic projections and we measured stimulated dopamine release in the central complex. ${ }^{5-8}$ Evoked release increased with increasing amounts of 
acetylcholine stimulation, and plateaued at $10 \mathrm{pmol}$ acetylcholine. The tissue content of dopamine in the CNS was also measured with capillary electrophoresis (CE). The effect of aging on dopamine was studied at three different ages in adult Drosophila control and Drosophila with Parkin-RNAi or RNF11-RNAi mutations.

The effect of aging on dopamine was first studied in control Drosophila to establish baseline conditions for studies in Parkinson's disease mutant flies. There was no significant effect of age on evoked dopamine or on dopamine tissue content. This result was consistent with a previous study that reported no change in the expression of $\mathrm{TH}$ in the brains of Drosophila as they aged. ${ }^{9}$ There was a significant effect of age when stimulations were repeated at 2-minute intervals as old flies had less decrease of evoked dopamine than mid-age flies. The result shows that unlike human brains where there is a decline in dopaminergic cell bodies and TH expression with age, Drosophila melanogaster do not have a significant change of dopamine levels in the CNS with age. The result is surprising as whole body levels of dopamine decline with age in Drosophila. ${ }^{10}$ Future studies should investigate if there is a decline of dopamine tissue content or stimulated release of dopamine in specific regions of the CNS. I established the baseline levels of dopamine tissue content and stimulated release with aging in control flies, and this method enables future studies of effects of aging in Drosophila models of age-related diseases such as Parkinson's disease and Alzheimer's disease. 
Next, effects of aging on dopamine in Parkin-RNAi or RNF11-RNAi Drosophila were investigated. Mutant Parkin is associated with human familial cases of Parkinson's disease and when expressed in Drosophila, it causes dopaminergic cell loss in Drosophila neurons. ${ }^{11}$ Also Parkin null mutant Drosophila have shrinkage of a subset of dopaminergic cells. ${ }^{12}$ RNF11 is another gene involved in Parkinson's disease, and is thought to be neuroprotective. ${ }^{13,14}$ Parkin-RNAi flies tested here did not have a significant change in dopamine tissue content with age, but RNF11-RNAi showed a significantly higher dopamine tissue content at old age. Both lines showed no significant difference in evoked dopamine release when stimulations were repeated at 2-minute intervals. This was different than control flies where old flies showed significantly less decrease during repeated stimulations compared to mid-age flies. The control flies therefore replenish more of the releasable pool of dopamine as they age, and the effect is lost in the Parkinson's disease model Drosophila.

Our study provides evidence that knocking down Parkin with RNAi causes no change in the concentration of stimulated dopamine release in the central complex or dopamine tissue content in the CNS of Drosophila, but does change dopamine dynamics at old age. Future studies should investigate if completely knocking out the Parkin or expressing human Parkin mutant gene has effects on dopamine levels. Parkin knockout flies have given mixed results on dopaminergic cell loss, and future studies of mutants that show a definite loss of dopaminergic cells will be tested to see if there is a significant defect in dopamine release and 
tissue content. ${ }^{11,15,16}$ Parkin null mutants with a complete knockout of Parkin have shown an effect in dopaminergic cells, however the effect of stimulated release is not studied. ${ }^{12}$ As my study sets a foundation for studying dopamine in adult Drosophila, it allows future investigations of changes in dopamine dynamics in Parkin null mutants that have previously shown to have smaller dopaminergic cells. ${ }^{12}$ Future research can therefore examine the effects of different Parkinson's disease mutations on the levels of dopamine and stimulated dopamine concentration in different brain regions. For example, two separate studies of Parkin mutants have reported dopaminergic cell loss in the PPL2ab or PAM regions. ${ }^{17,} 18$ The PPL2ab and PAM regions and regions where they send their projections, the calyx and the horizontal lobes, respectively, can be first investigated for any changes of dopamine signaling due to Parkin mutations. The study will advance the knowledge of how different brain regions are affected in Parkinson's disease model Drosophila and enable future studies of effects of drug treatments on dopamine neurochemistry.

There is no clear evidence of how much reduced TH expression in flies correlates with dopaminergic cell death or neuronal dysfunction. ${ }^{19}$ Direct measurements of levels of dopamine and tissue content with our method will allow better understanding of neurochemical changes in dopaminergic cells. Similarly, dopamine levels in Drosophila models of Alzheimer's and Huntington's disease can also be investigated as dopamine is suggested to play a role in the pathology of these diseases. ${ }^{20,21}$ Thus the method described here enables future 
studies of dopamine neurochemistry in Drosophila Parkinson's disease models as well as other disease models.

\subsection{Optimization of a FSCV waveform for in situ detection of octopamine}

The fourth chapter of this thesis describes optimization of a FSCV waveform for the detection of octopamine in situ. Octopamine is a major invertebrate neurotransmitter and plays important roles in learning, memory formation, ${ }^{22}$ locomotion ${ }^{23}$ and ovulation. ${ }^{24}$ Our lab had studied evoked dopamine and serotonin release in Drosophila melanogaster, but was not able to study octopamine. ${ }^{1,2}$ A FSCV waveform was optimized for in vitro detection of octopamine,${ }^{25}$ but the waveform was not suitable in situ. Octopamine oxidizes at a higher potential than dopamine or serotonin and the oxidation peak is shifted to the right close to the switching potential. The fast scan rate $(600 \mathrm{~V} / \mathrm{s})$ of the waveform optimized for in vitro octopamine detection and slowed electron transfer process in situ caused the peak shift to be even greater. ${ }^{26}$ Switching potential is where the signal is most unstable and non-faradaic signals can interfere with peaks close to the potential. By scanning up to $1.4 \mathrm{~V}$ and at a slower potential of $100 \mathrm{~V} / \mathrm{s}$, I was able to detect and characterize stimulated octopamine release in Drosophila larval VNC. ${ }^{27}$

Octopamine is a phenol and its oxidation product can further oxidize and create polymers at the electrode surface. This fouls the electrode and cause unstable responses. The new slower scan waveform lowered the effect of fouling by octopamine oxidation products as stable current responses were observed 
with repeated stimulations both in vitro and in situ. The method can be extended to other species that can cause electrode fouling, such as serotonin and tyramine. $^{28,29} \mathrm{FSCV}$ waveform optimization described here can also be used in the future to increase the sensitivity for other analytes or to increase selectivity towards a particular analyte.

Octopamine detection in vivo opens doors to study the neurochemical signaling of this molecule by FSCV. For example, we used the new waveform to show that RNF11-RNAi mutation in Drosophila larvae causes an increase in stimulated dopamine release, but not octopamine release, which shows that the mutation affects dopaminergic cells specifically. ${ }^{30}$ We can also study effects of other mutations such as ones in Drosophila vesicular monoamine transporter (dVMAT), and how they affect stimulated octopamine release with the method I have developed. ${ }^{31}$ Mutations in dVMAT affect larval locomotion or female fertility, both behaviors that are also modulated by octopamine. Therefore, future research can investigate how octopamine release and clearance kinetics are affected in dVMAT mutants to understand the link between behavior and octopamine neurochemistry. Optogenetically-stimulated octopamine release at the Drosophila neuromuscular junctions (NMJ) has been studied with amperometry as octopamine modulates the $\mathrm{NMJ}$ in arthopods. ${ }^{32}$ Amperometry is not a selective method as FSCV, and our method will allow selective measurements of evoked release in the $\mathrm{NMJ}$ in the future. 
Being able to detect endogenous octopamine in situ will allow better understanding of how octopamine neurotransmission mediates learning, memory, locomotion and ovulation. Future studies can also investigate if acetylcholine-stimulated octopamine release can be measured in Drosophila. Octopamine release mediated by nicotine has been measured in Drosophila before and since both nicotine and acetylcholine act on nAChRs, we expect to measure acetylcholine-stimulated octopamine release as well. ${ }^{33}$ The previous study used chronoamperometry to measure nicotine-stimulated octopamine release, and with FSCV we can make more selective measurements of the release. Our method will enable studies of stimulated octopamine release in any fly as release will be mediated by endogenous channels.

\subsection{Final Remarks}

This dissertation describes new methods to study stimulated dopamine release in Drosophila melanogaster larval and adult CNS. nAChR mediated dopamine release was measured in Drosophila larva and significant effects of nAChR mutations in neonicotinoid evoked response was measured. The study enables investigations of how nAChR mutations can mediate neurochemical responses in insects. Since nAChRs are natural ion channels, the new stimulation method can evoke dopamine release in wild type flies and other mutant flies that do not express special ion channels. For example, the method was used to study evoked dopamine release in adult Drosophila. Effects of aging on dopamine release in control flies and flies with Parkinson's disease mutations 
were also studied using acetylcholine stimulation. There was a significant effect of age when stimulations were repeated at short time intervals, and the effect was lost in Parkinson's disease mutant flies. This suggests that Parkinson's disease mutations affect how fast the releasable pool of dopamine restores in old adults. The study also establishes a baseline of dopamine signaling for aging studies in Drosophila. Future studies should investigate how dopamine dynamics change with age in Parkin null mutants and Drosophila expressing mutant human Parkin genes to see if the effects in dopamine neurochemistry are greater than with Parkin knockdown mutations. This thesis also describes optimization of the FSCV waveform for octopamine detection in Drosophila larval VNC. The waveform was optimized to decrease the effects of electrode fouling by oxidation products of octopamine for stable measurements of octopamine in situ. Red light stimulated octopamine in larval VNC was characterized. The research enables future studies of octopamine in Drosophila, such as investigations of how mutations in Drosophila affect octopamine neurotransmission. Similar methods of FSCV waveform optimization can be investigated for electroactive compounds that give unstable responses. FSCV allows rapid, sensitive and selective measurements of neurotransmitters. I have described methods here that can measure dopamine and octopamine in Drosophila larvae and adult CNS. The methods will enable future studies of how these neurotransmitters affect different functions in Drosophila, and how different mutations and disease models affect the neurotransmitters. 


\subsection{References}

[1] Borue, X., Cooper, S., Hirsh, J., Condron, B., and Venton, B. J. (2009) Quantitative evaluation of serotonin release and clearance in Drosophila, $J$ Neurosci Meth 179, 300-308.

[2] Vickrey, T. L., Condron, B., and Venton, B. J. (2009) Detection of endogenous dopamine changes in Drosophila melanogaster using fast-scan cyclic voltammetry, Analytical chemistry 81, 9306-9313.

[3] Xiao, N., and Venton, B. J. (2015) Characterization of dopamine releasable and reserve pools in Drosophila larvae using ATP/P2X(2)-mediated stimulation, J Neurochem 134, 445-454.

[4] Thany, S. H., Lenaers, G., Raymond-Delpech, V., Sattelle, D. B., and Lapied, B. (2007) Exploring the pharmacological properties of insect nicotinic acetylcholine receptors, Trends Pharmacol Sci 28, 14-22.

[5] Alekseyenko, O. V., Chan, Y. B., Li, R., and Kravitz, E. A. (2013) Single dopaminergic neurons that modulate aggression in Drosophila, $P$ Natl Acad Sci USA 110, 6151-6156.

[6] Kong, E. C., Woo, K., Li, H. Y., Lebestky, T., Mayer, N., Sniffen, M. R., Heberlein, U., Bainton, R. J., Hirsh, J., and Wolf, F. W. (2010) A Pair of Dopamine Neurons Target the D1-Like Dopamine Receptor DopR in the Central Complex to Promote Ethanol-Stimulated Locomotion in Drosophila, Plos One 5.

[7] Lebestky, T., Chang, J. S. C., Dankert, H., Zelnik, L., Kim, Y. C., Han, K. A., Wolf, F. W., Perona, P., and Anderson, D. J. (2009) Two Different Forms of Arousal in Drosophila Are Oppositely Regulated by the Dopamine D1 Receptor Ortholog DopR via Distinct Neural Circuits, Neuron 64, 522-536.

[8] Kim, Y. C., Lee, H. G., Seong, C. S., and Han, K. A. (2003) Expression of a D1 dopamine receptor dDA1/DmDOP1 in the central nervous system of Drosophila melanogaster, Gene Expr Patterns 3, 237-245.

[9] White, K. E., Humphrey, D. M., and Hirth, F. (2010) The dopaminergic system in the aging brain of Drosophila, Front Neurosci 4, 205. 
[10] Neckameyer, W. S., Woodrome, S., Holt, B., and Mayer, A. (2000) Dopamine and senescence in Drosophila melanogaster, Neurobiol Aging $21,145-152$.

[11] Sang, T. K., Chang, H. Y., Lawless, G. M., Ratnaparkhi, A., Mee, L., Ackerson, L. C., Maidment, N. T., Krantz, D. E., and Jackson, G. R. (2007) A Drosophila model of mutant human parkin-induced toxicity demonstrates selective loss of dopaminergic neurons and dependence on cellular dopamine, J Neurosci 27, 981-992.

[12] Greene, J. C., Whitworth, A. J., Kuo, I., Andrews, L. A., Feany, M. B., and Pallanck, L. J. (2003) Mitochondrial pathology and apoptotic muscle degeneration in Drosophila parkin mutants, P Natl Acad Sci USA 100, 4078-4083.

[13] Pranski, E., Van Sanford, C. D., Dalal, N., Orr, A. L., Karmali, D., Cooper, D. S., Gearing, M., Lah, J. J., Levey, A. I., and Betarbet, R. (2013) NF-kappa $B$ activity is inversely correlated to RNF11 expression in Parkinson's disease, Neurosci Lett 547, 16-20.

[14] Pranski, E. L., Dalal, N. V., Van Sanford, C., Herskowitz, J. H., Gearing, M., Lazo, C., Miller, G. W., Lah, J. J., Levey, A. I., and Betarbet, R. S. (2013) RING finger protein 11 (RNF11) modulates susceptibility to 6-OHDAinduced nigral degeneration and behavioral deficits through NF-kappa B signaling in dopaminergic cells, Neurobiol Dis 54, 264-279.

[15] Ortega-Arellano, H. F., Jimenez-Del-Rio, M., and Velez-Pardo, C. (2017) Minocycline protects, rescues and prevents knockdown transgenic parkin Drosophila against paraquat/iron toxicity: Implications for autosomic recessive juvenile parkinsonism, Neurotoxicology 60, 42-53.

[16] Greene, J. C., Whitworth, A. J., Kuo, I., Andrews, L. A., Feany, M. B., and Pallanck, L. J. (2003) Mitochondrial pathology and apoptotic muscle degeneration in Drosophila parkin mutants, Proc Natl Acad Sci U S A 100, 4078-4083.

[17] Kuo, S. Y., Wu, C. L., Hsieh, M. Y., Lin, C. T., Wen, R. K., Chen, L. C., Chen, Y. H., Yu, Y. W., Wang, H. D., Su, Y. J., Lin, C. J., Yang, C. Y., Guan, H. Y., Wang, P. Y., Lan, T. H., and Fu, T. F. (2015) PPL2ab neurons restore sexual responses in aged Drosophila males through dopamine, Nat Commun 6. 
[18] Wang, C., Lu, R. F., Ouyang, X. Z., Ho, M. W. L., Chia, W., Yu, F. W., and Lim, K. L. (2007) Drosophila overexpressing parkin R275W mutant exhibits dopaminergic neuron degeneration and mitochondrial abnormalities, J Neurosci 27, 8563-8570.

[19] Navarro, J. A., Hessner, S., Yenisetti, S. C., Bayersdorfer, F., Zhang, L., Voigt, A., Schneuwly, S., and Botella, J. A. (2014) Analysis of dopaminergic neuronal dysfunction in genetic and toxin-induced models of Parkinson's disease in Drosophila, $J$ Neurochem 131, 369-382.

[20] Martorana, A., and Koch, G. (2014) "Is dopamine involved in Alzheimer's disease?", Front Aging Neurosci 6.

[21] Cepeda, C., Murphy, K. P. S., Parent, M., and Levine, M. S. (2014) The role of dopamine in huntington's disease, Prog Brain Res 211, 235-254.

[22] Schwaerzel, M., Monastirioti, M., Scholz, H., Friggi-Grelin, F., Birman, S., and Heisenberg, M. (2003) Dopamine and octopamine differentiate between aversive and appetitive olfactory memories in Drosophila, $J$ Neurosci 23, 10495-10502.

[23] Saraswati, S., Fox, L. E., Soll, D. R., and Wu, C. F. (2004) Tyramine and octopamine have opposite effects on the locomotion of Drosophila larvae, J Neurobiol 58, 425-441.

[24] Hardie, S. L., Zhang, J. X., and Hirsh, J. (2007) Trace Amines differentially regulate adult locomotor activity, cocaine sensitivity, and female fertility in Drosophila melanogaster, Dev Neurobiol 67, 1396-1405.

[25] Cooper, S. E., and Venton, B. J. (2009) Fast-scan cyclic voltammetry for the detection of tyramine and octopamine, Anal Bioanal Chem 394, 329-336.

[26] Amatore, C., Saveant, J. M., and Tessier, D. (1983) Charge-Transfer at Partially Blocked Surfaces - a Model for the Case of Microscopic Active and Inactive Sites, J Electroanal Chem 147, 39-51.

[27] Pyakurel, P., Champaloux, E. P., and Venton, B. J. (2016) Fast-Scan Cyclic Voltammetry (FSCV) Detection of Endogenous Octopamine in Drosophila melanogaster Ventral Nerve Cord, Acs Chemical Neuroscience 7, 11121119. 
[28] Guell, A. G., Meadows, K. E., Unwin, P. R., and Macpherson, J. V. (2010) Trace voltammetric detection of serotonin at carbon electrodes: comparison of glassy carbon, boron doped diamond and carbon nanotube network electrodes, Phys Chem Chem Phys 12, 10108-10114.

[29] Floyer, B., and Jensen, N. (2007) Stabilization of the voltammetric response of organic analytes with self-passivating electrode reactions: Synergistic effect of surfactants and high buffer strength, J Electroanal Chem 601, 153-160.

[30] Champaloux, E. P. (2016) Dopamine Kinetics in a Drosophila Model of Parkinson Disease [dissertation], University of Virginia, Charlottesville.

[31] Grygoruk, A., Chen, A., Martin, C. A., Lawal, H. O., Fei, H., Gutierrez, G., Biedermann, T., Najibi, R., Hadi, R., Chouhan, A. K., Murphy, N. P., Schweizer, F. E., Macleod, G. T., Maidment, N. T., and Krantz, D. E. (2014) The Redistribution of Drosophila Vesicular Monoamine Transporter Mutants from Synaptic Vesicles to Large Dense-Core Vesicles Impairs Amine-Dependent Behaviors, J Neurosci 34, 6924-6937.

[32] Majdi, S., Berglund, E. C., Dunevall, J., Oleinick, A. I., Amatore, C., Krantz, D. E., and Ewing, A. G. (2015) Electrochemical Measurements of Optogenetically Stimulated Quantal Amine Release from Single Nerve Cell Varicosities in Drosophila Larvae, Angew Chem Int Edit 54, 13609-13612.

[33] Fuenzalida-Uribe, N., Meza, R. C., Hoffmann, H. A., Varas, R., and Campusano, J. M. (2013) nAChR-induced octopamine release mediates the effect of nicotine on a startle response in Drosophila melanogaster, $J$ Neurochem 125, 281-290. 


\section{Bibliography}

Agrawal, Dasaradhi, Mohmmed, Malhotra, Bhatnagar, \& Mukherjee. (2003). RNA interference: Biology, mechanism, and applications. Microbiology and Molecular Biology Reviews, 67(4), 657-+. doi: 10.1128/Mmbr.67.4.657685.2003

Alekseyenko, Chan, Li, \& Kravitz. (2013). Single dopaminergic neurons that modulate aggression in Drosophila. Proceedings of the National Academy of Sciences of the United States of America, 110(15), 6151-6156. doi: 10.1073/pnas. 1303446110

Amatore, Saveant, \& Tessier. (1983). Charge-Transfer at Partially Blocked Surfaces - a Model for the Case of Microscopic Active and Inactive Sites. Journal of Electroanalytical Chemistry, 147(1-2), 39-51. doi: Doi 10.1016/S0022-0728(83)80055-2

Anderson, Betarbet, Gearing, Gulcher, Hicks, Stefansson, ... Levey. (2007). PARK10 candidate RNF11 is expressed by vulnerable neurons and localizes to Lewy bodies in Parkinson disease brain. Journal of Neuropathology and Experimental Neurology, 66(10), 955-964.

Axelrod, \& Saavedra. (1977). Octopamine. Nature, 265(5594), 501-504.

Azam, Winzer-Serhan, Chen, \& Leslie. (2002). Expression of neuronal nicotinic acetylcholine receptor subunit mRNAs within midbrain dopamine neurons. Journal of Comparative Neurology, 444(3), 260-274. doi: 10.1002/cne.10138

Bailey, Martin, \& Downer. (1982). Simultaneous Determination of Dopamine, Norepinephrine, Tyramine and Octopamine by Reverse-Phase HighPerformance Liquid-Chromatography with Electrochemical Detection. Journal of Liquid Chromatography, 5(12), 2435-2452. doi: Doi 10.1080/01483918208067645

Bainton, Tsai, Singh, Moore, Neckameyer, \& Heberlein. (2000). Dopamine modulates acute responses to cocaine, nicotine and ethanol in Drosophila. Current Biology, 10(4), 187-194. doi: Doi 10.1016/S0960-9822(00)00336-5

Balfanz, Strunker, Frings, \& Baumann. (2005). A family of octopamine [corrected] receptors that specifically induce cyclic AMP production or $\mathrm{Ca} 2+$ release in Drosophila melanogaster. J Neurochem, 93(2), 440-451. doi: 10.1111/j.1471-4159.2005.03034.x 
Bartus, Dean, Beer, \& Lippa. (1982). The Cholinergic Hypothesis of Geriatric Memory Dysfunction. Science, 217(4558), 408-417. doi: Doi 10.1126/Science.7046051

Bass, Grinevich, Vance, Sullivan, Bonin, \& Budygin. (2010). Optogenetic control of striatal dopamine release in rats. $J$ Neurochem, 114(5), 1344-1352. doi: 10.1111/j.1471-4159.2010.06850.x

Bellen, Tong, \& Tsuda. (2010). TIMELINE 100 years of Drosophila research and its impact on vertebrate neuroscience: a history lesson for the future. Nature Reviews Neuroscience, 11(7), 514-+. doi: 10.1038/nrn2839

Benke, \& Breer. (1989). Comparison of Acetylcholine and Alpha-Bungarotoxin Binding-Sites in Insects and Vertebrates. Comparative Biochemistry and Physiology C-Pharmacology Toxicology \& Endocrinology, 94(1), 71-80. doi: Doi 10.1016/0742-8413(89)90146-1

Benzer. (1967). Behaavioral Mutant of Dorsophila Isolated by Countercurrent Distribution. Proc Natl Acad Sci U S A, 58(3), 1112-1119.

Bernheimer, Birkmayer, Hornykiewicz, Jellinger, \& Seitelberger. (1973). Brain dopamine and the syndromes of Parkinson and Huntington. Clinical, morphological and neurochemical correlations. J Neurol Sci, 20(4), 415455.

Berry. (2004). Mammalian central nervous system trace amines. Pharmacologic amphetamines, physiologic neuromodulators. Journal of Neurochemistry, 90(2), 257-271. doi: 10.1111/j.1471-4159.2004.02501.x

Bhimani. (2014). Understanding the Burden on Caregivers of People with Parkinson's: A Scoping Review of the Literature. Rehabil Res Pract, 2014, 718527. doi: $10.1155 / 2014 / 718527$

Borue, Condron, \& Venton. (2010). Both synthesis and reuptake are critical for replenishing the releasable serotonin pool in Drosophila. J Neurochem, 113(1), 188-199. doi: 10.1111/j.1471-4159.2010.06588.x

Borue, Cooper, Hirsh, Condron, \& Venton. (2009). Quantitative evaluation of serotonin release and clearance in Drosophila. Journal of Neuroscience Methods, 179(2), 300-308. doi: 10.1016/j.jneumeth.2009.02.013

Brand, \& Perrimon. (1993). Targeted Gene-Expression as a Means of Altering Cell Fates and Generating Dominant Phenotypes. Development, 118(2), 401-415. 
Breer. (1981). Properties of putative nicotinic and muscarinic cholinergic receptors in the central nervous system of Locusta migratoria. Neurochem Int, 3(1), 43-52.

Breer, \& Sattelle. (1987). Molecular-Properties and Functions of Insect Acetylcholine-Receptors. Journal of Insect Physiology, 33(11), 771-790. doi: Doi 10.1016/0022-1910(87)90025-4

Brisch, Saniotis, Wolf, Bielau, Bernstein, Steiner, ... Gos. (2014). The role of dopamine in schizophrenia from a neurobiological and evolutionary perspective: old fashioned, but still in vogue. Front Psychiatry, 5, 47. doi: 10.3389/fpsyt.2014.00047

Brown, Ihara, Buckingham, Matsuda, \& Sattelle. (2006). Neonicotinoid insecticides display partial and super agonist actions on native insect nicotinic acetylcholine receptors. Journal of Neurochemistry, 99(2), 608615. doi: 10.1111/j.1471-4159.2006.04084.x

Bunin, \& Wightman. (1998). Quantitative evaluation of 5-hydroxytryptamine (serotonin) neuronal release and uptake: An investigation of extrasynaptic transmission. Journal of Neuroscience, 18(13), 4854-4860.

Bymaster FP. (2002). Fluoxetine, but not other selective serotonin uptake inhibitors, increases norepinephrine and dopamine extracellular levels in prefrontal cortex. Psychopharmacology (Berl), 160(4), 353-361.

Campusano, Su, Jiang, Sicaeros, \& O'Dowd. (2007). nAChR-Mediated calcium responses and plasticity in Drosophila Kenyon cells. Developmental Neurobiology, 67(11), 1520-1532. doi: 10.1002/dneu.20527

Cano-de-la-Cuerda, Perez-de-Heredia, Miangolarra-Page, Munoz-Hellin, \& Fernandez-de-las-Penas. (2010). Is There Muscular Weakness in Parkinson's Disease? American Journal of Physical Medicine \& Rehabilitation, 89(1), 70-76. doi: 10.1097/PHM.0b013e3181a9ed9b

Carlsson, Henning, Lindberg, Martinson, Trolin, Waldeck, \& Wickberg. (1978). On the disulfiram-like effect of coprine, the pharmacologically active principle of Coprinus atramentarius. Acta Pharmacol Toxicol (Copenh), 42(4), 292-297.

Cepeda, Murphy, Parent, \& Levine. (2014). The role of dopamine in huntington's disease. Dopamine, 211, 235-254. doi: 10.1016/B978-0-444-634252.00010-6 
Cha, Kim, Park, Lee, Kim, Lee, . . . Cho. (2005). Parkin negatively regulates JNK pathway in the dopaminergic neurons of Drosophila. Proc Natl Acad Sci U $S$ A, 102(29), 10345-10350. doi: 10.1073/pnas.0500346102

Champaloux. (2016). Dopamine Kinetics in a Drosophila Model of Parkinson Disease [dissertation]. (Dissertation), University of Virginia, Charlottesville.

Cheon, Ryu, Kim, \& Cho. (2005). The homozygosity for 10-repeat in allele at dopamine transporter gene and dopamine transporter density in Korean children with attention deficit hyperactivity disorder: relating to treatment response to methylphenidate. European Neuropsychopharmacology, 15(1), 95-101. doi: 10.1016/j.euroneuro.2004.06.004

Clark, Dever, Dever, Xu, Rehder, Sosa, \& Baro. (2004). Arthropod 5-HT2 receptors: a neurohormonal receptor in decapod crustaceans that displays agonist independent activity resulting from an evolutionary alteration to the DRY motif. J Neurosci, 24(13), 3421-3435. doi: 10.1523/JNEUROSCI.0062-04.2004

Clark, Dodson, Jiang, Cao, Huh, Seol, ... Guo. (2006). Drosophila pink1 is required for mitochondrial function and interacts genetically with parkin. Nature, 441(7097), 1162-1166. doi: 10.1038/nature04779

Colas, Launay, Kellermann, Rosay, \& Maroteaux. (1995). Drosophila 5-HT2 serotonin receptor: coexpression with fushi-tarazu during segmentation. Proc Natl Acad Sci U S A, 92(12), 5441-5445.

Cole, Carney, McClung, Willard, Taylor, \& Hirsh. (2005). Two functional but noncomplementing Drosophila tyrosine decarboxylase genes: distinct roles for neural tyramine and octopamine in female fertility. $\mathrm{J} \mathrm{Biol} \mathrm{Chem,}$ 280(15), 14948-14955. doi: 10.1074/jbc.M414197200

Cooley, Kelley, \& Spradling. (1988). Insertional mutagenesis of the Drosophila genome with single P elements. Science, 239(4844), 1121-1128.

Cooper, \& Venton. (2009). Fast-scan cyclic voltammetry for the detection of tyramine and octopamine. Analytical and Bioanalytical Chemistry, 394(1), 329-336. doi: 10.1007/s00216-009-2616-0

Corey, Quick, Davidson, Lester, \& Guastella. (1994). A Cocaine-Sensitive Drosophila Serotonin Transporter - Cloning, Expression, and Electrophysiological Characterization. Proceedings of the National Academy of Sciences of the United States of America, 91(3), 1188-1192. doi: Doi 10.1073/Pnas.91.3.1188 
Corringer, Le Novere, \& Changeux. (2000). Nicotinic receptors at the amino acid level. Annual Review of Pharmacology and Toxicology, 40, 431-458. doi: DOI 10.1146/annurev.pharmtox.40.1.431

Couton, Mauss, Yunusov, Diegelmann, Evers, \& Landgraf. (2015). Development of Connectivity in a Motoneuronal Network in Drosophila Larvae. Current Biology, 25(5), 568-576. doi: 10.1016/j.cub.2014.12.056

D'andrea, Nordera, Pizzolato, Bolner, Colavito, Flaibani, \& Leon. (2010). Trace amine metabolism in Parkinson's disease: Low circulating levels of octopamine in early disease stages. Neuroscience Letters, 469(3), 348351. doi: 10.1016/j.neulet.2009.12.025

de Lau, \& Breteler. (2006). Epidemiology of Parkinson's disease. Lancet Neurol, 5(6), 525-535. doi: 10.1016/S1474-4422(06)70471-9

Demchyshyn, Pristupa, Sugamori, Barker, Blakely, Wolfgang, ... Niznik. (1994). Cloning, Expression, and Localization of a Chloride-Facilitated, CocaineSensitive Serotonin Transporter from Drosophila-Melanogaster. Proceedings of the National Academy of Sciences of the United States of America, 91(11), 5158-5162. doi: Doi 10.1073/Pnas.91.11.5158

Deng, Dodson, Huang, \& Guo. (2008). The Parkinson's disease genes pink1 and parkin promote mitochondrial fission and/or inhibit fusion in Drosophila. Proceedings of the National Academy of Sciences of the United States of America, 105(38), 14503-14508. doi: 10.1073/pnas.0803998105

Denno, Privman, \& Venton. (2015). Analysis of Neurotransmitter Tissue Content of Drosophila melanogaster in Different Life Stages. Acs Chemical Neuroscience, 6(1), 117-123. doi: 10.1021/cn500261e

Ding, Guzman, Peterson, Goldberg, \& Surmeier. (2010). Thalamic Gating of Corticostriatal Signaling by Cholinergic Interneurons. Neuron, 67(2), 294307. doi: 10.1016/j.neuron.2010.06.017

Ding, \& Sachs. (1999). Single channel properties of P2X2 purinoceptors. J Gen Physiol, 113(5), 695-720.

Doherty, van de Warrenburg, Peralta, Silveira-Moriyama, Azulay, Gershanik, \& Bloem. (2011). Postural deformities in Parkinson's disease. Lancet Neurology, 10(6), 538-549. doi: 10.1016/S1474-4422(11)70067-9

Donly, \& Caveney. (2005). A transporter for phenolamine uptake in the arthropod CNS. Arch Insect Biochem Physiol, 59(3), 172-183. doi: 10.1002/arch.20063 
Duffy. (2002). GAL4 system in Drosophila: A fly geneticist's Swiss army knife. Genesis, 34(1-2), 1-15. doi: 10.1002/gene.1015

Eckermann, Feld, Shaw, \& Meade. (2010). Electrochemistry of redox-active selfassembled monolayers. Coordination Chemistry Reviews, 254(15-16), 1769-1802. doi: 10.1016/j.ccr.2009.12.023

El Hassani, Dacher, Gary, Lambin, Gauthier, \& Armengaud. (2008). Effects of sublethal doses of acetamiprid and thiamethoxam on the behavior of the honeybee (Apis mellifera). Archives of Environmental Contamination and Toxicology, 54(4), 653-661. doi: 10.1007/s00244-007-9071-8

Eusebio, Azulay, Ceccaldi, Girard, Mundler, \& Guedj. (2012). Voxel-based analysis of whole-brain effects of age and gender on dopamine transporter SPECT imaging in healthy subjects. European Journal of Nuclear Medicine and Molecular Imaging, 39(11), 1778-1783. doi: $10.1007 / \mathrm{s} 00259-012-2207-8$

Exley, \& Cragg. (2008). Presynaptic nicotinic receptors: a dynamic and diverse cholinergic filter of striatal dopamine neurotransmission. British Journal of Pharmacology, 153, S283-S297. doi: 10.1038/sj.bjp.0707510

Fang, Vickrey, \& Venton. (2011). Analysis of Biogenic Amines in a Single Drosophila Larva Brain by Capillary Electrophoresis with Fast-Scan Cyclic Voltammetry Detection. Analytical Chemistry, 83(6), 2258-2264. doi: 10.1021/ac103092z

Farooqui. (2012). Review of octopamine in insect nervous system. Open access insect physiology, 4, 1-17.

Faust, Gehrke, Yang, Yang, Beal, \& Lu. (2009). Neuroprotective effects of compounds with antioxidant and anti-inflammatory properties in a Drosophila model of Parkinson's disease. Bmc Neuroscience, 10. doi: Artn 109 10.1186/1471-2202-10-109

Feany, \& Bender. (2000). A Drosophila model of Parkinson's disease. Nature, 404(6776), 394-398. doi: Doi 10.1038/35006074

Fearnley, \& Lees. (1991a). Ageing and Parkinson's disease: substantia nigra regional selectivity. Brain, 114 ( Pt 5), 2283-2301.

Fearnley, \& Lees. (1991b). Aging and Parkinsons-Disease - Substantia-Nigra Regional Selectivity. Brain, 114, 2283-2301. doi: DOI 10.1093/brain/114.5.2283 
Fields, \& Woodring. (1991). Octopamine Mobilization of Lipids and Carbohydrates in the House Cricket, Acheta-Domesticus. Journal of Insect Physiology, 37(3), 193-199. doi: Doi 10.1016/0022-1910(91)90069-C

Fiore, Dolan, Strausfeld, \& Hirth. (2015). Evolutionarily conserved mechanisms for the selection and maintenance of behavioural activity. Philosophical Transactions of the Royal Society B-Biological Sciences, 370(1684). doi: ARTN 2015005310.1098/rstb.2015.0053

Floyer, \& Jensen. (2007). Stabilization of the voltammetric response of organic analytes with self-passivating electrode reactions: Synergistic effect of surfactants and high buffer strength. Journal of Electroanalytical Chemistry, 601(1-2), 153-160. doi: 10.1016/j.jelechem.2006.11.001

Forno. (1996). Neuropathology of Parkinson's disease. Journal of Neuropathology and Experimental Neurology, 55(3), 259-272. doi: Doi 10.1097/00005072-199603000-00001

Fredieu, \& Mahowald. (1989). Glial Interactions with Neurons during Drosophila Embryogenesis. Development, 106(4), 739-\&.

Fuenzalida-Uribe, Meza, Hoffmann, Varas, \& Campusano. (2013). nAChRinduced octopamine release mediates the effect of nicotine on a startle response in Drosophila melanogaster. J Neurochem, 125(2), 281-290. doi: $10.1111 /$ jnc. 12161

Fuke, Suo, Takahashi, Koike, Sasagawa, \& Ishiura. (2001). The VNTR polymorphism of the human dopamine transporter (DAT1) gene affects gene expression. Pharmacogenomics J, 1(2), 152-156.

Fukuto. (1990). Mechanism of Action of Organophosphorus and Carbamate Insecticides. Environmental Health Perspectives, 87, 245-254.

Fuzeau-Braesch. (1983). Determination of octopamine using high pressure liquid chromatography and electrochemical detection: studies of the brain of the cricket Locusta migratoria cinerascens. Agressologie: revue internationale de physio-biologie et de pharmacologie appliquees aux effets de l'agression, 24(8), 377-379.

Gasser. (1997). Advances in the genetics of movement disorders: Implications for molecular diagnosis. Journal of Neurology, 244(6), 341-348. doi: DOI $10.1007 / \mathrm{s} 004150050100$

Gauglitz, \& Pfluger. (2001). Cholinergic transmission via central synapses in the locust nervous system. Journal of Comparative Physiology a- 
Neuroethology Sensory Neural and Behavioral Physiology, 187(10), 825836. doi: DOI 10.1007/s00359-001-0253-y

Georghiou. (1994). Principles of Insecticide Resistance Management. Phytoprotection, 75, 51-59.

Gibb. (1991). Neuropathology of the substantia nigra. Eur Neurol, 31 Suppl 1, 4859.

Gill, Savolainen, Young, Zwart, Sher, \& Millar. (2011). Agonist activation of alpha 7 nicotinic acetylcholine receptors via an allosteric transmembrane site. Proceedings of the National Academy of Sciences of the United States of America, 108(14), 5867-5872. doi: 10.1073/pnas.1017975108

Giordano, Rendina, Peluso, \& Furia. (2002). RNAi triggered by symmetrically transcribed transgenes in Drosophila melanogaster. Genetics, 160(2), 637-648.

Gotti, Moretti, Bohr, Ziabreva, Vailati, Longhi, . . Court. (2006). Selective nicotinic acetylcholine receptor subunit deficits identified in Alzheimer's disease, Parkinson's disease and dementia with Lewy bodies by immunoprecipitation. Neurobiology of Disease, 23(2), 481-489. doi: 10.1016/j.nbd.2006.04.005

Govind, Vezina, \& Green. (2009). Nicotine-induced upregulation of nicotinic receptors: underlying mechanisms and relevance to nicotine addiction. Biochem Pharmacol, 78(7), 756-765. doi: 10.1016/j.bcp.2009.06.011

Greene, Whitworth, Kuo, Andrews, Feany, \& Pallanck. (2003). Mitochondrial pathology and apoptotic muscle degeneration in Drosophila parkin mutants. Proceedings of the National Academy of Sciences of the United States of America, 100(7), 4078-4083. doi: 10.1073/pnas.0737556100

Groger, Kolb, Schafer, \& Klose. (2014). Dopamine reduction in the substantia nigra of Parkinson's disease patients confirmed by in vivo magnetic resonance spectroscopic imaging. PLoS One, 9(1), e84081. doi: 10.1371/journal.pone.0084081

Grygoruk, Chen, Martin, Lawal, Fei, Gutierrez, . . Krantz. (2014). The Redistribution of Drosophila Vesicular Monoamine Transporter Mutants from Synaptic Vesicles to Large Dense-Core Vesicles Impairs AmineDependent Behaviors. Journal of Neuroscience, 34(20), 6924-6937. doi: 10.1523/Jneurosci.0694-14.2014

Guell, Meadows, Unwin, \& Macpherson. (2010). Trace voltammetric detection of serotonin at carbon electrodes: comparison of glassy carbon, boron doped 
diamond and carbon nanotube network electrodes. Physical Chemistry Chemical Physics, 12(34), 10108-10114. doi: 10.1039/c0cp00675k

Hafizi, Kruk, \& Stamford. (1990). Fast Cyclic Voltammetry - Improved Sensitivity to Dopamine with Extended Oxidation Scan Limits. Journal of Neuroscience Methods, 33(1), 41-49. doi: Doi 10.1016/01650270(90)90080-Y

Hales, Korey, Larracuente, \& Roberts. (2015). Genetics on the Fly: A Primer on the Drosophila Model System. Genetics, 201(3), 815-842. doi: 10.1534/genetics.115.183392

Hammer, \& Menzel. (1998). Multiple sites of associative odor learning as revealed by local brain microinjections of octopamine in honeybees. Learning \& Memory, 5(1-2), 146-156.

Hardie, Zhang, \& Hirsh. (2007). Trace amines differentially regulate adult locomotor activity, cocaine sensitivity, and female fertility in Drosophila melanogaster. Dev Neurobiol, 67(10), 1396-1405. doi:

10.1002/dneu.20459

Harrow, \& Sattelle. (1983). Acetylcholine-Receptors on the Cell Body Membrane of Giant Interneuron-2 in the Cockroach, Periplaneta-Americana. Journal of Experimental Biology, 105(Jul), 339-350.

Hasselmo. (2006). The role of acetylcholine in learning and memory. Current Opinion in Neurobiology, 16(6), 710-715. doi: DOI 10.1016/j.conb.2006.09.002

Hawley, Tatawawadi, Piekarski, \& Adams. (1967). Electrochemical studies of the oxidation pathways of catecholamines. J Am Chem Soc, 89(2), 447-450.

Heien, Phillips, Stuber, Seipel, \& Wightman. (2003). Overoxidation of carbon-fiber microelectrodes enhances dopamine adsorption and increases sensitivity. Analyst, 128(12), 1413-1419. doi: 10.1039/b307024g

Heinrich, Hedwig, \& Elsner. (1997). Cholinergic activation of stridulatory behaviour in the grasshopper Omocestus viridulus (L). Journal of Experimental Biology, 200(9), 1327-1337.

Hendricks, Finn, Panckeri, Chavkin, Williams, Sehgal, \& Pack. (2000). Rest in Drosophila is a sleep-like state. Neuron, 25(1), 129-138. doi: Doi 10.1016/S0896-6273(00)80877-6

Herbin, Simonis, Reveret, Hackert, Libourel, Eugene, . . . Vidal. (2016). Dopamine Modulates Motor Control in a Specific Plane Related to 
Support. Plos One, 11(5). doi: ARTN e0155058

10.1371/journal.pone.0155058

Hermanns. (2011). Weathering the storm: living with Parkinson's disease. $J$ Christ Nurs, 28(2), 76-82; quiz 83-74.

Hilario, Turner, \& Blendy. (2012). Reward sensitization: effects of repeated nicotine exposure and withdrawal in mice. Neuropsychopharmacology, 37(12), 2661-2670. doi: 10.1038/npp.2012.130

Hornig. (1998). Addressing comorbidity in adults with attentiondeficit/hyperactivity disorder. Journal of Clinical Psychiatry, 59, 69-75.

Hoy. (1988). Arthropod Brain - Its Evolution, Development, Structure, and Functions - Gupta,Ap. Science, 240(4852), 668-668. doi: DOI 10.1126/science.240.4852.668

Huffman, \& Venton. (2009). Carbon-fiber microelectrodes for in vivo applications. Analyst, 134(1), 18-24. doi: 10.1039/b807563h

Jackson, Dietz, \& Wightman. (1995). Fast-Scan Cyclic Voltammetry of 5Hydroxytryptamine. Analytical Chemistry, 67(6), 1115-1120. doi: Doi 10.1021/Ac00102a015

Jankovic. (2005). Motor fluctuations and dyskinesias in Parkinson's disease: clinical manifestations. Movement Disorders, 20, S11-S16. doi: $10.1002 /$ mds.20458

Jankovic. (2008). Parkinson's disease: clinical features and diagnosis. Journal of Neurology Neurosurgery and Psychiatry, 79(4), 368-376. doi: 10.1136/jnnp.2007.131045

Javoy-Agid, Hirsch, Dumas, Duyckaerts, Mallet, \& Agid. (1990). Decreased tyrosine hydroxylase messenger RNA in the surviving dopamine neurons of the substantia nigra in Parkinson's disease: an in situ hybridization study. Neuroscience, 38(1), 245-253.

Jeibmann, \& Paulus. (2009). Drosophila melanogaster as a Model Organism of Brain Diseases. International Journal of Molecular Sciences, 10(2), 407440. doi: 10.3390/ijms 10020407

Jensen, Mikkelsen, Frolund, Brauner-Osborne, Falch, \& Krogsgaard-Larsen. (2003). Carbamoylcholine homologs: Novel and potent agonists at neuronal nicotinic acetylcholine receptors. Molecular Pharmacology, 64(4), 865-875. doi: DOI 10.1124/mol.64.4.865 
Jepson, Brown, \& Sattelle. (2006). The actions of the neonicotinoid imidacloprid on cholinergic neurons of Drosophila melanogaster. Invert Neurosci, 6(1), 33-40. doi: 10.1007/s10158-005-0013-8

Jeschke, Nauen, Schindler, \& Elbert. (2011). Overview of the Status and Global Strategy for Neonicotinoids. Journal of Agricultural and Food Chemistry, 59(7), 2897-2908. doi: 10.1021/jf101303g

Kao, \& Karlin. (1986). Acetylcholine-Receptor Binding-Site Contains a Disulfide Cross-Link between Adjacent Half-Cystinyl Residues. Journal of Biological Chemistry, 261(18), 8085-8088.

Kawagoe, Zimmerman, \& Wightman. (1993). Principles of Voltammetry and Microelectrode Surface-States. Journal of Neuroscience Methods, 48(3), 225-240. doi: Doi 10.1016/0165-0270(93)90094-8

Ke, Lu, \& Cheng. (2006). A strategy for the determination of dopamine at a bare glassy carbon electrode: $\mathrm{p}$-Phenylenediamine as a nucleophile. Electrochemistry Communications, 8(9), 1514-1520. doi: 10.1016/j.elecom.2006.07.007

Kim, Lee, Seong, \& Han. (2003). Expression of a D1 dopamine receptor dDA1/DmDOP1 in the central nervous system of Drosophila melanogaster. Gene Expression Patterns, 3(2), 237-245. doi: 10.1016/S1567-133x(02)00098-4

Kimura-Kuroda, Komuta, Kuroda, Hayashi, \& Kawano. (2012). Nicotine-Like Effects of the Neonicotinoid Insecticides Acetamiprid and Imidacloprid on Cerebellar Neurons from Neonatal Rats. Plos One, 7(2). doi: ARTN e32432.10.1371/journal.pone.0032432

Kitada, Asakawa, Hattori, Matsumine, Yamamura, Minoshima, ... Shimizu. (1998). Mutations in the parkin gene cause autosomal recessive juvenile parkinsonism. Nature, 392(6676), 605-608.

Klapoetke, Murata, Kim, Pulver, Birdsey-Benson, Cho, ... Boyden. (2014). Independent optical excitation of distinct neural populations. Nat Methods, 11(3), 338-346. doi: 10.1038/nmeth.2836

Klink, d'Exaerde, Zoli, \& Changeux. (2001). Molecular and physiological diversity of nicotinic acetylcholine receptors in the midbrain dopaminergic nuclei. Journal of Neuroscience, 21(5), 1452-1463.

Klink, de Kerchove d'Exaerde, Zoli, \& Changeux. (2001). Molecular and physiological diversity of nicotinic acetylcholine receptors in the midbrain dopaminergic nuclei. J Neurosci, 21(5), 1452-1463. 
Kohler, Cierpinsky, Kronenberg, \& Adli. (2016). The serotonergic system in the neurobiology of depression: Relevance for novel antidepressants. Journal of Psychopharmacology, 30(1), 13-22. doi: 10.1177/0269881115609072

Kong, Woo, Li, Lebestky, Mayer, Sniffen, ... Wolf. (2010). A Pair of Dopamine Neurons Target the D1-Like Dopamine Receptor DopR in the Central Complex to Promote Ethanol-Stimulated Locomotion in Drosophila. Plos One, 5(3). doi: ARTN e9954

10.1371/journal.pone.0009954

Koo, An, Park, Kim, \& Kim. (2014). Regional susceptibilities to 12 insecticides of melon and cotton aphid, Aphis gossypii (Hemiptera: Aphididae) and a point mutation associated with imidacloprid resistance. Crop Protection, 55, 91-97. doi: 10.1016/j.cropro.2013.09.010

Koob, \& Simon. (2009). The Neurobiology of Addiction: Where We Have Been and Where We Are Going. Journal of Drug Issues, 39(1), 115-132.

Kuo, Wu, Hsieh, Lin, Wen, Chen, ... Fu. (2015). PPL2ab neurons restore sexual responses in aged Drosophila males through dopamine. Nature Communications, 6. doi: ARTN 7490

$10.1038 /$ ncomms 8490

Lansdell, Collins, Goodchild, \& Millar. (2012). The Drosophila nicotinic acetylcholine receptor subunits Dalpha5 and Dalpha7 form functional homomeric and heteromeric ion channels. BMC Neurosci, 13, 73. doi: $10.1186 / 1471-2202-13-73$

Lawal, Terrell, Lam, Djapri, Jang, Hadi, . . Krantz. (2014). Drosophila modifier screens to identify novel neuropsychiatric drugs including aminergic agents for the possible treatment of Parkinson's disease and depression. Molecular Psychiatry, 19(2), 235-242. doi: 10.1038/mp.2012.170

Lebestky, Chang, Dankert, Zelnik, Kim, Han, . . Anderson. (2009). Two Different Forms of Arousal in Drosophila Are Oppositely Regulated by the Dopamine D1 Receptor Ortholog DopR via Distinct Neural Circuits. Neuron, 64(4), 522-536. doi: 10.1016/j.neuron.2009.09.031

Lee, \& O'dowd. (1999). Fast excitatory synaptic transmission mediated by nicotinic acetylcholine receptors in Drosophila neurons. Journal of Neuroscience, 19(13), 5311-5321. 
Lee, Weiner, \& Trendelenburg. (1967). The uptake of tyramine and formation of octopamine in normal and tachyphylactic rat atria. J Pharmacol Exp Ther, 155(2), 211-222.

Lindstrom, Ryding, Ahnlide, Bosson, Traskman-Bendz, \& Rosen. (2005). Impulsivity related to serotonin transporter measured by SPECT in suicide attempters. Nordic Journal of Psychiatry, 59(5), 414-415.

Littleton, \& Ganetzky. (2000). Ion channels and synaptic organization: Analysis of the Drosophila genome. Neuron, 26(1), 35-43. doi: Doi 10.1016/S08966273(00)81135-6

Lucking, Durr, Bonifati, Vaughan, De Michele, Gasser, . . Stu. (2000).

Association between early-onset Parkinson's disease and mutations in the parkin gene. New England Journal of Medicine, 342(21), 1560-1567. doi:

Doi 10.1056/Nejm200005253422103

Lummis, \& Sattelle. (1985). Binding of N-[propionyl-3H]propionylated alphabungarotoxin and L-[benzilic-4,4'-3H] quinuclidinyl benzilate to CNS extracts of the cockroach Periplaneta americana. Comp Biochem Physiol C, 80(1), 75-83.

Majdi, Berglund, Dunevall, Oleinick, Amatore, Krantz, \& Ewing. (2015). Electrochemical Measurements of Optogenetically Stimulated Quantal Amine Release from Single Nerve Cell Varicosities in Drosophila Larvae. Angew Chem Int Ed Engl. doi: 10.1002/anie.201506743

Mansvelder, Keath, \& McGehee. (2002). Synaptic mechanisms underlie nicotineinduced excitability of brain reward areas. Neuron, 33(6), 905-919.

Martin, \& Krantz. (2014). Drosophila melanogaster as a genetic model system to study neurotransmitter transporters. Neurochemistry International, 73, 7188. doi: 10.1016/j.neuint.2014.03.015

Martin-Ruiz, Piggot, Gotti, Lindstrom, Mendelow, Siddique, ... Court. (2000). Alpha and beta nicotinic acetylcholine receptors subunits and synaptophysin in putamen from Parkinson's disease. Neuropharmacology, 39(13), 2830-2839. doi: Doi 10.1016/S0028-3908(00)00110-6

Martorana, \& Koch. (2014). "Is dopamine involved in Alzheimer's disease?". Frontiers in Aging Neuroscience, 6. doi: ARTN 252 10.3389/fnagi.2014.00252

Matsuda, Buckingham, Kleier, Rauh, Grauso, \& Sattelle. (2001). Neonicotinoids: insecticides acting on insect nicotinic acetylcholine receptors. Trends in 
Pharmacological Sciences, 22(11), 573-580. doi: Doi 10.1016/S01656147(00)01820-4

McGeer, Itagaki, Akiyama, \& McGeer. (1988). Rate of cell death in parkinsonism indicates active neuropathological process. Ann Neurol, 24(4), 574-576. doi: 10.1002/ana.410240415

Mcgeer, Mcgeer, \& Suzuki. (1977). Aging and Extrapyramidal Function. Archives of Neurology, 34(1), 33-35.

Monastirioti. (2003). Distinct octopamine cell population residing in the CNS abdominal ganglion controls ovulation in Drosophila melanogaster. Developmental Biology, 264(1), 38-49. doi: 10.1016/j.ydbio.2003.07.019

Morens, Davis, Grandinetti, Ross, Popper, \& White. (1996). Epidemiologic observations on Parkinson's disease: Incidence and mortality in a prospective study of middle-aged men. Neurology, 46(4), 1044-1050.

Morgan, \& Weber. (1984). Noise and signal-to-noise ratio in electrochemical detectors. Anal Chem, 56(13), 2560-2567.

Mota-Sanchez, Hollingworth, Grafius, \& Moyer. (2006). Resistance and crossresistance to neonicotinoid insecticides and spinosad in the Colorado potato beetle, Leptinotarsa decemlineata (Say) (Coleoptera : Chrysomelidae). Pest Management Science, 62(1), 30-37. doi: 10.1002/ps. 1120

Musacchio. (1964). Effects of disulfiram on tissue norepinephrine content and subcellular distribution of dopamine, tyramine and their b-hydroxylated metabolites. Life Sciences, 3, 769-775.

Nathanson, Hunnicutt, Kantham, \& Scavone. (1993). Cocaine as a NaturallyOccurring Insecticide. Proceedings of the National Academy of Sciences of the United States of America, 90(20), 9645-9648. doi: Doi 10.1073/Pnas.90.20.9645

Navarro, Hessner, Yenisetti, Bayersdorfer, Zhang, Voigt, . . Botella. (2014). Analysis of dopaminergic neuronal dysfunction in genetic and toxininduced models of Parkinson's disease in Drosophila. Journal of Neurochemistry, 131(3), 369-382. doi: 10.1111/jnc.12818

Navera, Suzuki, Tamiya, Takeuchi, \& Karube. (1993). Nafion-Coated CarbonFiber for Acetylcholine and Choline Sensors. Electroanalysis, 5(1), 17-22. doi: DOI 10.1002/elan.1140050105 
Neckameyer, Woodrome, Holt, \& Mayer. (2000). Dopamine and senescence in Drosophila melanogaster. Neurobiology of Aging, 21(1), 145-152. doi: Doi 10.1016/S0197-4580(99)00109-8

Nishikawa, \& Kidokoro. (1999). Octopamine inhibits synaptic transmission at the larval neuromuscular junction in Drosophila melanogaster. Brain Research, 837(1-2), 67-74. doi: Doi 10.1016/S0006-8993(99)01676-5

Noisternig. (2000). Carbon fibre composites as stay cables for bridges. Applied Composite Materials, 7(2-3), 139-150. doi: Doi 10.1023/A:1008946132034

North. (2002). Molecular physiology of P2X receptors. Physiol Rev, 82(4), 10131067. doi: 10.1152/physrev.00015.2002

Ohnishi. (1977). Spontaneous and ethyl methanesulfonate-induced mutations controlling viability in Drosophila melanogaster. III. Heterozygous effect of polygenic mutations. Genetics, 87(3), 547-556.

Oldenziel, \& Westerink. (2005). Improving glutamate microsensors by optimizing the composition of the redox hydrogel. Analytical Chemistry, 77(17), 55205528. doi: 10.1021/ac0580013

Orchard, Ramirez, \& Lange. (1993). A Multifunctional Role for Octopamine in Locust Flight. Annual Review of Entomology, 38, 227-249. doi: Doi 10.1146/Annurev.En.38.010193.001303

Ortega-Arellano, Jimenez-Del-Rio, \& Velez-Pardo. (2017). Minocycline protects, rescues and prevents knockdown transgenic parkin Drosophila against paraquat/iron toxicity: Implications for autosomic recessive juvenile parkinsonism. Neurotoxicology, 60, 42-53. doi:

10.1016/j.neuro.2017.03.002

Pan-Montojo, \& Reichmann. (2014). Considerations on the role of environmental toxins in idiopathic Parkinson's disease pathophysiology. Transl Neurodegener, 3, 10. doi: 10.1186/2047-9158-3-10

Pandey, \& Nichols. (2011). Human Disease Models in Drosophila melanogaster and the Role of the Fly in Therapeutic Drug Discovery. Pharmacological Reviews, 63(2), 411-436. doi: 10.1124/pr.110.003293

Paxon, Powell, Lee, Han, \& Ewing. (2005). Microcolumn separation of amine metabolites in the fruit fly. Anal Chem, 77(16), 5349-5355. doi: $10.1021 / \mathrm{ac} 050474 \mathrm{~m}$

Perry, Heckel, McKenzie, \& Batterham. (2008). Mutations in D alpha 1 or D beta 2 nicotinic acetylcholine receptor subunits can confer resistance to 
neonicotinoids in Drosophila melanogaster. Insect Biochemistry and Molecular Biology, 38(5), 520-528. doi: 10.1016/j.ibmb.2007.12,007

Perry, McKenzie, \& Batterham. (2007). A Dalpha6 knockout strain of Drosophila melanogaster confers a high level of resistance to spinosad. Insect Biochem Mol Biol, 37(2), 184-188. doi: 10.1016/j.ibmb.2006.11.009

Pesah, Pham, Burgess, Middlebrooks, Verstreken, Zhou, ... Mardon. (2004). Drosophila parkin mutants have decreased mass and cell size and increased sensitivity to oxygen radical stress. Development, 131(9), 21832194. doi: $10.1242 / \mathrm{dev} .01095$

Peters, \& Kreulen. (1986). Fast and Slow Synaptic Potentials Produced in a Mammalian Sympathetic-Ganglion by Colon Distension. Proceedings of the National Academy of Sciences of the United States of America, 83(6), 1941-1944. doi: DOI 10.1073/pnas.83.6.1941

Pifl, Kish, \& Hornykiewicz. (2012). Thalamic noradrenaline in Parkinson's disease: Deficits suggest role in motor and non-motor symptoms. Movement Disorders, 27(13), 1618-1624. doi: 10.1002/mds.25109

Pihel, Schroeder, \& Wightman. (1994). Rapid and Selective Cyclic Voltammetric Measurements of Epinephrine and Norepinephrine as a Method to Measure Secretion from Single Bovine Adrenal-Medullary Cells. Analytical Chemistry, 66(24), 4532-4537. doi: DOI 10.1021/ac00096a021

Pihel, Walker, \& Wightman. (1996). Overoxidized polypyrrole-coated carbon fiber microelectrodes for dopamine measurements with fast-scan cyclic voltammetry. Analytical Chemistry, 68(13), 2084-2089. doi: DOI 10.1021/ac960153y

Plumlee. (2004). Clinical Veterinary Toxicology. St. Louis, Mo.: Mosby.

Polotis, \& Niccolini. (2015). Serotonin in Parkinson's disease. Behavioural Brain Research, 277, 136-145. doi: 10.1016/j.bbr.2014.07.037

Polymeropoulos, Lavedan, Leroy, Ide, Dehejia, Dutra, ... Nussbaum. (1997). Mutation in the alpha-synuclein gene identified in families with Parkinson's disease. Science, 276(5321), 2045-2047. doi: DOI 10.1126/science.276.5321.2045

Ponchon, Cespuglio, Gonon, Jouvet, \& Pujol. (1979). Normal pulse polarography with carbon fiber electrodes for in vitro and in vivo determination of catecholamines. Anal Chem, 51(9), 1483-1486. 
Porzgen, Park, Hirsh, Sonders, \& Amara. (2001). The antidepressant-sensitive dopamine transporter in Drosophila melanogaster: A primordial carrier for catecholamines. Molecular Pharmacology, 59(1), 83-95.

Pranski, Dalal, Van Sanford, Herskowitz, Gearing, Lazo, . . Betarbet. (2013). RING finger protein 11 (RNF11) modulates susceptibility to 6-OHDAinduced nigral degeneration and behavioral deficits through NF-kappa B signaling in dopaminergic cells. Neurobiology of Disease, 54, 264-279. doi: 10.1016/j.nbd.2012.12.018

Pranski, Van Sanford, Dalal, Orr, Karmali, Cooper, . . Betarbet. (2013). NFkappa B activity is inversely correlated to RNF11 expression in Parkinson's disease. Neuroscience Letters, 547, 16-20. doi: 10.1016/j.neulet.2013.04.056

Privman, \& Venton. (2015). Comparison of Dopamine Kinetics in the Larval Drosophila Ventral Nerve Cord and Protocerebrum with Improved Optogenetic Stimulation. J Neurochem. doi: 10.1111/jnc.13286

Pyakurel, Champaloux, \& Venton. (2016). Fast-Scan Cyclic Voltammetry (FSCV) Detection of Endogenous Octopamine in Drosophila melanogaster Ventral Nerve Cord. Acs Chemical Neuroscience, 7(8), 1112-1119. doi: 10.1021/acschemneuro.6b00070

Pyakurel, Privman Champaloux, \& Venton. (2016). Fast-Scan Cyclic Voltammetry (FSCV) Detection of Endogenous Octopamine in Drosophila melanogaster Ventral Nerve Cord. ACS Chem Neurosci. doi: 10.1021/acschemneuro.6b00070

Quinn. (1987). Acetylcholinesterase - Enzyme Structure, Reaction Dynamics, and Virtual Transition-States. Chemical Reviews, 87(5), 955-979. doi: DOI $10.1021 / \mathrm{cr} 00081 \mathrm{a} 005$

Rakovic, Grunewald, Seibler, Ramirez, Kock, Orolicki, . . Klein. (2010). Effect of endogenous mutant and wild-type PINK1 on Parkin in fibroblasts from Parkinson disease patients. Human Molecular Genetics, 19(16), 31243137. doi: $10.1093 / \mathrm{hmg} / \mathrm{ddq} 215$

Rice, \& Cragg. (2004). Nicotine amplifies reward-related dopamine signals in striatum. Nature Neuroscience, 7(6), 583-584. doi: 10.1038/nn1244

Roeder. (1999). Octopamine in invertebrates. Prog Neurobiol, 59(5), 533-561.

Rohrbough, \& Broadie. (2002). Electrophysiological analysis of synaptic transmission in central neurons of Drosophila larvae. Journal of Neurophysiology, 88(2), 847-860. doi: 10.1152/jn01010.2001 
Ross, \& Venton. (2014). Sawhorse Waveform Voltammetry for Selective Detection of Adenosine, ATP, and Hydrogen Peroxide. Analytical Chemistry, 86(15), 7486-7493. doi: 10.1021/ac501229c

Salkoff, Baker, Butler, Covarrubias, Pak, \& Wei. (1992). An Essential Set of K(+) Channels Conserved in Flies, Mice and Humans. Trends in Neurosciences, 15(5), 161-166. doi: Doi 10.1016/0166-2236(92)90165-5

Salvaterra, \& Foders. (1979). [2-Alpha-Bungarotoxin-I-125 and [Quinuclidinylbenzilate-H-3 Binding in Central Nervous Systems of Different Species. Journal of Neurochemistry, 32(5), 1509-1517. doi: DOI 10.1111/j.1471-4159.1979.tb11092.x

Samaha, Yau, Yang, \& Robinson. (2005). Rapid delivery of nicotine promotes behavioral sensitization and alters its neurobiological impact. Biological Psychiatry, 57(4), 351-360. doi: DOI 10.1016/j.biopsych.2004.11.040

Sanchez-Diaz, Rosales-Bravo, Reyes-Taboada, Covarrubias, Narvaez-Padilla, \& Reynaud. (2015). The Esg Gene Is Involved in Nicotine Sensitivity in Drosophila melanogaster. Plos One, 10(7). doi: ARTN e0133956 10.1371/journal.pone.0133956

Sandler, Ruthven, Goodwin, Reynolds, Rao, \& Coppen. (1979). Deficient Production of Tyramine and Octopamine in Cases of Depression. Nature, 278(5702), 357-358. doi: Doi 10.1038/278357a0

Sang, Chang, Lawless, Ratnaparkhi, Mee, Ackerson, . . Jackson. (2007). A Drosophila model of mutant human parkin-induced toxicity demonstrates selective loss of dopaminergic neurons and dependence on cellular dopamine. Journal of Neuroscience, 27(5), 981-992. doi: 10.1523/Jneurosci.4810-06.2007

Saraswati, Fox, Soll, \& Wu. (2004). Tyramine and octopamine have opposite effects on the locomotion of Drosophila larvae. Journal of Neurobiology, 58(4), 425-441. doi: 10.1002/neu.10298

Scherzer, Jensen, Gullans, \& Feany. (2003). Gene expression changes presage neurodegeneration in a Drosophila model of Parkinson's disease. Human Molecular Genetics, 12(19), 2457-2466. doi: 10.1093/hmg/ddg265

Scholz-Kornehl, \& Schwarzel. (2016). Circuit Analysis of a Drosophila Dopamine Type 2 Receptor That Supports Anesthesia-Resistant Memory. Journal of Neuroscience, 36(30), 7936-7945. doi: 10.1523/Jneurosci.4475-15.2016

Schwaerzel, Monastirioti, Scholz, Friggi-Grelin, Birman, \& Heisenberg. (2003). Dopamine and octopamine differentiate between aversive and appetitive 
olfactory memories in Drosophila. Journal of Neuroscience, 23(33), 10495-10502.

Selcho, Pauls, El Jundi, Stocker, \& Thum. (2012). The role of octopamine and tyramine in Drosophila larval locomotion. J Comp Neurol, 520(16), 37643785. doi: $10.1002 / \mathrm{cne} .23152$

Self, Guthrie, \& Hodgson. (1964). Metabolism of Nicotine by Tobacco-Feeding Insects. Nature, 204, 300-301.

Shaw, Cirelli, Greenspan, \& Tononi. (2000). Correlates of sleep and waking in Drosophila melanogaster. Science, 287(5459), 1834-1837. doi: DOI 10.1126/science.287.5459.1834

Shin, Rogers, Devoto, Bjorklund, \& Carta. (2014). Noradrenaline neuron degeneration contributes to motor impairments and development of $\mathrm{L}$ DOPA-induced dyskinesia in a rat model of Parkinson's disease. Experimental Neurology, 257, 25-38. doi: 10.1016/j.expneurol.2014.04.011

Sin, Michels, \& Nollen. (2014). Genetic screens in Caenorhabditis elegans models for neurodegenerative diseases. Biochim Biophys Acta, 1842(10), 1951-1959. doi: 10.1016/j.bbadis.2014.01.015

Spatola, \& Wider. (2014). Genetics of Parkinson's disease: the yield. Parkinsonism Relat Disord, 20 Suppl 1, S35-38. doi: 10.1016/S13538020(13)70011-7

Steppuhn, Gase, Krock, Halitschke, \& Baldwin. (2004). Nicotine's defensive function in nature. Plos Biology, 2(8), 1074-1080. doi: ARTN e21710.1371/journal.pbio.0020217

Swamy, \& Venton. (2007). Subsecond detection of physiological adenosine concentrations using fast-scan cyclic voltammetry. Analytical Chemistry, 79(2), 744-750. doi: 10.1021/ac061820i

Sweeney, Hidalgo, de Belle, \& Keshishian. (2011). Dissection of adult Drosophila brains. Cold Spring Harb Protoc, 2011(12), 1472-1474. doi: 10.1101/pdb.prot066878

Taber, \& Hurley. (2014). Volume Transmission in the Brain: Beyond the Synapse. Journal of Neuropsychiatry and Clinical Neurosciences, 26(1).

Taillebois, Beloula, Quinchard, Jaubert-Possamai, Daguin, Servent, ... TricoireLeignel. (2014). Neonicotinoid Binding, Toxicity and Expression of 
Nicotinic Acetylcholine Receptor Subunits in the Aphid Acyrthosiphon pisum. Plos One, 9(5). doi: ARTN e9666910.1371/journal.pone.0096669

Thany, Lenaers, Raymond-Delpech, Sattelle, \& Lapied. (2007). Exploring the pharmacological properties of insect nicotinic acetylcholine receptors. Trends in Pharmacological Sciences, 28(1), 14-22. doi: 10.1016/j.tips.2006.11.006

Tomizawa, Talley, Maltby, Durkin, Medzihradszky, Burlingame, ... Casida. (2007). Mapping the elusive neonicotinoid binding site. Proc Natl Acad Sci U S A, 104(21), 9075-9080. doi: 10.1073/pnas.0703309104

Triarhou. (2002). Dopamine and Parkinson's disease - Introduction. Dopaminergic Neuron Transplantation in the Weaver Mouse Model of Parkinson's Disease, 517, 1-14.

Ueno, Suzuki, Naganos, Ofusa, Horiuchi, \& Saitoe. (2017). Coincident postsynaptic activity gates presynaptic dopamine release to induce plasticity in Drosophila mushroom bodies. Elife, 6. doi: ARTN e21076

10.7554/eLife.21076

Valente, Abou-Sleiman, Caputo, Muqit, Harvey, Gispert, . . Wood. (2004). Hereditary early-onset Parkinson's disease caused by mutations in PINK1. Science, 304(5674), 1158-1160. doi: 10.1126/science.1096284

Valente, das Neves, \& Oliveira. (2012). Epigenetic engineering to reverse the Parkinson's expression state. Parkinsonism \& Related Disorders, 18(6), 717-721. doi: 10.1016/j.parkreldis.2012.04.018

Venken, \& Bellen. (2005). Emerging technologies for gene manipulation in Drosophila melanogaster (vol 6, pg 167, 2005). Nature Reviews Genetics, 6(4), 340-340. doi: 10.1038/nrg1600

Venton, \& Wightman. (2003). Psychoanalytical electrochemistry: Dopamine and behavior. Analytical Chemistry, 75(19), 414a-421a. doi: DOI 10.1021/ac031421c

Vickrey, Condron, \& Venton. (2009). Detection of endogenous dopamine changes in Drosophila melanogaster using fast-scan cyclic voltammetry. Anal Chem, 81(22), 9306-9313. doi: 10.1021/ac901638z

Vickrey, Xiao, \& Venton. (2013). Kinetics of the Dopamine Transporter in Drosophila Larva. Acs Chemical Neuroscience, 4(5), 832-837. doi: $10.1021 / \mathrm{cn} 400019 \mathrm{q}$ 
Vomel, \& Wegener. (2008). Neuroarchitecture of aminergic systems in the larval ventral ganglion of Drosophila melanogaster. PLoS One, 3(3), e1848. doi: 10.1371/journal.pone.0001848

Wallace. (1976). The biosynthesis of octopamine--characterization of lobster tyramine beta-hydroxylase. J Neurochem, 26(4), 761-770.

Wang, Lu, Ouyang, Ho, Chia, Yu, \& Lim. (2007). Drosophila overexpressing parkin R275W mutant exhibits dopaminergic neuron degeneration and mitochondrial abnormalities. Journal of Neuroscience, 27(32), 8563-8570. doi: 10.1523/Jneurosci.0218-07.2007

White, Humphrey, \& Hirth. (2010). The dopaminergic system in the aging brain of Drosophila. Front Neurosci, 4, 205. doi: 10.3389/fnins.2010.00205

Whitworth. (2011). Drosophila Models of Parkinson's Disease. Advances in Genetics, Vol 73, 73, 1-50. doi: 10.1016/B978-0-12-380860-8.00001-X

Whitworth, Theodore, Greene, Benes, Wes, \& Pallanck. (2005). Increased glutathione S-transferase activity rescues dopaminergic neuron loss in a Drosophila model of Parkinson's disease. Proceedings of the National Academy of Sciences of the United States of America, 102(22), 80248029. doi: 10.1073/pnas.0501078102

Wiedemann, Garris, Near, \& Wightman. (1992). Effect of Chronic Haloperidol Treatment on Stimulated Synaptic Overflow of Dopamine in the Rat Striatum. Journal of Pharmacology and Experimental Therapeutics, 261(2), 574-579.

Williams, Steffens, \& Bacon. (1970). Bending Behavior and Tensile Strength of Carbon Fibers. Journal of Applied Physics, 41(12), 4893-\&. doi: Doi 10.1063/1.1658559

Williamson, \& Hiesinger. (2010). Preparation of developing and adult Drosophila brains and retinae for live imaging. J Vis $\operatorname{Exp(37).~doi:~10.3791/1936~}$

Winklhofer, \& Haass. (2010). Mitochondrial dysfunction in Parkinson's disease. Biochimica Et Biophysica Acta-Molecular Basis of Disease, 1802(1), 2944. doi: 10.1016/j.bbadis.2009.08.013

Wu, Ma, Pierzchala, Wu, Yang, Mai, ... Schmidt-Glenewinkel. (2005). The Drosophila acetylcholine receptor subunit D alpha 5 is part of an alphabungarotoxin binding acetylcholine receptor. Journal of Biological Chemistry, 280(22), 20987-20994. doi: DOI 10.1074/jbc.M409639200 
Xiao, Privman, \& Venton. (2014). Optogenetic Control of Serotonin and Dopamine Release in Drosophila Larvae. Acs Chemical Neuroscience, 5(8), 666-673. doi: 10.1021/cn500044b

Xiao, \& Venton. (2015). Characterization of dopamine releasable and reserve pools in Drosophila larvae using ATP/P2X(2)-mediated stimulation. Journal of Neurochemistry, 134(3), 445-454. doi: 10.1111/jnc.13148

Yamamoto, \& Seto. (2014). Dopamine Dynamics and Signaling in Drosophila: An Overview of Genes, Drugs and Behavioral Paradigms. Experimental Animals, 63(2), 107-119.

Yuan, \& Lee. (2007). Suppression of excitatory cholinergic synaptic transmission by Drosophila dopamine D1-like receptors. European Journal of Neuroscience, 26(9), 2417-2427. doi: 10.1111/j.1460-9568.2007.05870.x

Zachek, Park, Takmakov, Wightman, \& McCarty. (2010). Microfabricated FSCVcompatible microelectrode array for real-time monitoring of heterogeneous dopamine release. Analyst, 135(7), 1556-1563. doi: 10.1039/c0an00114g

Zheng, Felder, Connor, \& Poo. (1994). Turning of Nerve Growth Cones Induced by Neurotransmitters. Nature, 368(6467), 140-144. doi: DOI $10.1038 / 368140 \mathrm{a0}$

Zhou, Huang, Kim, Lin, Meng, Han, . . Rao. (2012). Molecular Genetic Analysis of Sexual Rejection: Roles of Octopamine and Its Receptor OAMB in Drosophila Courtship Conditioning. Journal of Neuroscience, 32(41), 14281-14287. doi: 10.1523/JNEUROSCI.0517-12.2012

Zhou, Liang, \& Dani. (2001). Endogenous nicotinic cholinergic activity regulates dopamine release in the striatum. Nature Neuroscience, 4(12), 1224-1229. doi: DOI 10.1038/nn769

Zhou, Rao, \& Rao. (2008). A subset of octopaminergic neurons are important for Drosophila aggression. Nature Neuroscience, 11(9), 1059-1067. doi: 10.1038/nn.2164

Zimprich, Biskup, Leitner, Lichtner, Farrer, Lincoln, . . Gasser. (2004). Mutations in LRRK2 cause autosomal-dominant Parkinsonism with pleomorphic pathology. Neuron, 44(4), 601-607. doi: DOI 10.1016/j.neuron.2004.11.005

Zwarts, Versteven, \& Callaerts. (2012). Genetics and neurobiology of aggression in Drosophila. Fly, 6(1), 35-48. doi: 10.4161/fly.19249 